\title{
Catálogo bibliográfico dos taxa superiores da ordem Lepidoptera ${ }^{1}$
}

\author{
Olaf H. H. Mielke ${ }^{2} \&$ Mirna M. Casagrande ${ }^{2}$ \\ ${ }^{1}$ Contribuição número 1609 do Departamento de Zoologia, Universidade Federal do Paraná. \\ ${ }^{2}$ Departamento de Zoologia, Universidade Federal do Paraná. Caixa Postal 19020, 81531-980 Curitiba, Paraná, Brasil. \\ E-mail: omhesp@ufpr.br; mibras@ufpr.br
}

\begin{abstract}
Catalogue of the higher taxa in the order Lepidoptera. A list with the names of the superior categories of the Lepidoptera order is presented occasioning the establishment of some synonymies. The first part contains the sequence of names in chronological order of publication as well as relevant subsequent citations; the second part contains the phylogenetic scheme of Lepidoptera proposed by HePPNER (2003); the third part contains the synonymies in accordance with HEPPNER's system; the fourth part contains the phylogenetic scheme of HEPpNER based on the synonymies established and the fifth part contains an index with the names of the authors and date of publication. The following names are revalidated and the respective synonyms are established: Lepidopteroidea Heymons, 1915, syn.: Amphiesmenoptera Kiriakoff, 1948; Micropterygina Herrich-Schäffer, 1856, syn.: Zeugloptera Chapman, 1916; Paleolepidoptera Packard, 1895, syn.: Dacnonypha Hinton, 1946; Archifrenata Küppers \& Speidel, V-1980, syn.: Neopseustina Davis \& Nielsen, XI-1980; Hepialites Latreille, 1809, syn.: Exoporia Common, 1975; Anisoneuria Karsch, 1898, syn.: Heteroneura Tillyard, 1918; Nepticulae Chapman, 1898, syn.: Nepticulina Meyrick, 1928; Adeliformes Lameere, 1936, syn.: Incurvariina Börner, 1939; Tineae Scopoli, 1763, syn.: Tineina Stainton, 1857; Zygaenides Leach, 1815, syn.: Sesiina Heppner, 1995; Bombyces Scopoli, 1763, syn.: Bombycina Stainton, 1857.

KEY WORDS. Neotropical; taxonomy.
\end{abstract}

RESUMO. Apresenta-se uma lista com os nomes das categorias superiores da ordem Lepidoptera, fato que ocasionou o estabelecimento de algumas sinonímias. Contém na primeira parte a sequência dos nomes em ordem cronológica de publicação, assim como relevantes citações subsequentes; na segunda parte o esquema filogenético de Lepidoptera proposto por HEPPNER (2003); na terceira parte as sinonímias de acordo com o sistema de HEPPNER; na quarta parte o esquema filogenético de HEPPNER baseado nas sinonímias estabelecidas e na quinta parte um índice dos nomes com os autores e a data de publicação. Os seguintes nomes são revalidados e respectivos sinônimos estabelecidos: Lepidopteroidea Heymons, 1915, syn.: Amphiesmenoptera Kiriakoff, 1948; Micropterygina Herrich-Schäffer, 1856, syn.: Zeugloptera Chapman, 1916; Paleolepidoptera Packard, 1895, syn.: Dacnonypha Hinton, 1946; Archifrenata Küppers \& Speidel, V-1980, syn.: Neopseustina Davis \& Nielsen, XI-1980; Hepialites Latreille, 1809, syn.: Exoporia Common, 1975; Anisoneuria Karsch, 1898, syn.: Heteroneura Tillyard, 1918; Nepticulae Chapman, 1898, syn.: Nepticulina Meyrick, 1928; Adeliformes Lameere, 1936, syn.: Incurvariina Börner, 1939; Tineae Scopoli, 1763, syn.: Tineina Stainton, 1857; Zygaenides Leach, 1815, syn.: Sesiina Heppner, 1995; Bombyces Scopoli, 1763, syn.: Bombycina Stainton, 1857.

PALAVRAS CHAVE. Neotropical; taxonomia.

\begin{abstract}
SUMÁRIO

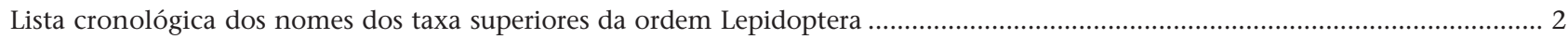

Esquema filogenético da ordem Lepidoptera conforme Heppner (2003) ...................................................................................26

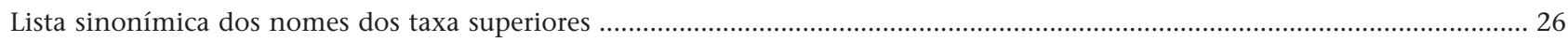

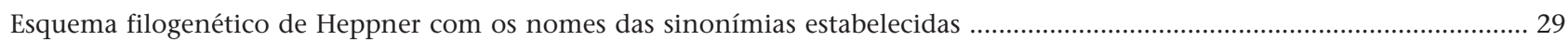

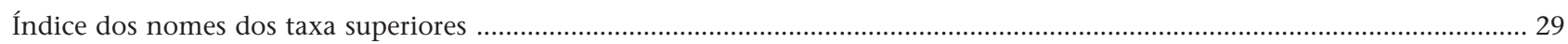

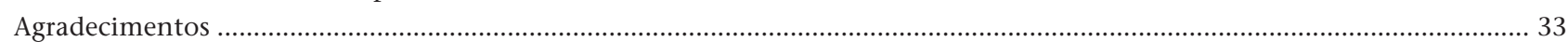

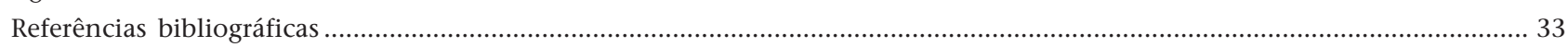


A sistemática é uma ciência das mais problemáticas, principalmente devida à falta de ordem e conceitos dos nomes publicados. Embora o "Código Internacional de Nomenclatura Zoológica" apresente as regras para a definição de nomes de grupos de família, gênero e espécie, ele não abrange os nomes das categorias superiores. Em consequência muitos taxa são propostos e aceitos, embora perfeitos sinônimos, simplesmente por desconhecimento dos nomes mais antigos ou também por serem, muitas vezes, publicados em livros e/ou revistas de pouca circulação. Outro agravante é o não regsitro destes nomes pelos indexadores tradicionais.

Para sanar esta falha e tentar uniforemizar os nomes dos taxa superiores na ordem Lepidoptera, embora não mandatário no "Código Internacional de Nomenclatura Zoológica", fez-se um levantamento na literatura consultada durante pesquisas sobre a fauna neotropical ao longo dos anos, na esperança de que a lista seja a mais completa possível. Também são mencionadas citações posteriores aos nomes listados, assim como aqueles que na sequencia foram reconceituados ao status original, ou seja, os que foram estabelecidos como nomes de categorias superiores ou inferiores, além de nomes com "aspecto" de categoria superior, com ou sem status definido, e as grafias errônea. Para completar a lista, foram incluidos os nomes referentes à superordem que incluem as ordens Lepidoptera e Trichoptera.

Apresenta-se na primeira parte, em ordem cronológica de publicação, os nomes dos taxa com as referências originais e subsequentes mais significativas encontradas; na segunda parte o mais recente esquema filogenético dos grupos superiores da ordem Lepidoptera, proposto por Heppner (2003); na terceira parte uma lista sinonímica dos nomes de acordo com o mesmo esquema; na quarta parte um esquema filogenético onde cada taxon é denominado pelo nome mais antigo, baseado nas sinonímias aqui estabelecidas, considerando-se que nestes casos os o princípio da prioridade também deva ser aplicado; na quinta parte um índice alfabético de todos os nomes encontrados, com autores e datas de publicação; e nas sexta e sétima partes os agradecementos e as referências bibliográficas, respectivamente.

Adotando-se o esquema filogenético de HeppNer (2003), ao todo 11 nomes foram validados e 11 foram sinonimizados, entre os 412 nomes relacionados.

Infelizmente não foi possível localizar as citações originais de Macrolepidoptera e Microlepidoptera, provavelmente estabelecidos por HERRICH-SCHÄFFER, ou outro autor contemporâneo; também a descrição de Sorbentia Haeckel, 1866, mencionada com esta data por HANDLIRSCH (1925), só encontrada na oitava edição (HAECKel 1889).

Na lista bibliográfica dos nomes optou-se por incluir a referência bibliográfica de modo abreviado, simplesmente para facilitar o leitor. A referência completa encontra-se nas "Referências Bibliográficas".

Espera-se desta forma contribuir para uma sistemática dos grupos superiores de Lepidoptera, ao menos em termos dos nomes. Caso os colegas encontrem outros nomes e/ou datas, fato bastante provável, solicita-se informar para a inclusão em eventual contribuição complementar.

\section{LISTA CRONOLÓGICA DOS NOMES DOS TAXA SUPERIORES DA ORDEM LEPIDOPTERA}

\section{Lepidopteroidea Heymons, 1915}

Lepidopteroidea Heymons, 1915, in zur Strassen. Brehms Tierleben, $4^{\mathrm{a}}$ ed, p. 202, grupo incluindo Panorpata, Mecaptera, Trichoptera, Lepidoptera.- Krausse \& Wolff, 1919. Arch. Natg. 86A(3): 166; grupo de ordens, incluindo Trichoptera, Lepidoptera.

Amphiesmenoptera Kiriakoff, 1948. Biol. Jaarboek, Antwerpen, 15, p. 127; ordem, incluindo Zeugloptera, Trichoptera, Lepidoptera.- Hennig, 1953. Beitr. Ent. 3, Sonderh., p. 46, 76; ordem, incluindo Trichoptera e Lepidoptera.

Amphiesménoptères [sic]; Kiriakoff, 1952. Ann. Soc. zool. Belg. 83: 95, 96 [= Amphiesmenoptera].

\section{Lepidoptera Linnaeus, 1758}

Lepidoptera Linnaeus, 1758. Syst. nat. $10^{\text {a }}$ ed., p. 341, 343, 458; ordem, incluindo vários gêneros.- Linnaeus, 1767. Syst. nat. $12^{a}$ ed., p. 744.- Scopoli, 1763. Ent. Carniolica, p. 142.Stoll, 1782, in: Cramer. Uitland. Kap. 4, Essai, p. 1.- Retzius, 1783. Caroli De Geer gesp. nov. insect., p. III, IV.Jablonsky, 1783. Naturs. bek. in- und ausländ. Ins., p. 189, 195; syn.: Glossata.- Roemer, 1789. Gen. ins. Linn. Fabr., p. 16.- Gmelin, 1790. C. Linné. Syst. nat. $13^{\mathrm{a}}$ ed., 1, p. 225.- Thunberg, 1791. Charact. Gen. Ins., p. 34.- Latreille, 1796. Préc. caract., p. 140.- Latreille, 1802. Hist. nat., gén. part. Crust. Ins. 2, p. 350, 365.- Latreille, 1805. Hist. nat., gén. part. Crust. Ins. 14, p. 69.- Latreille, 1806. Gen. Crust. Ins. 1, p. 171.-Latreille, 1809. Gen. Crust. Ins. 4, p. 183; syn.: Glossata.- Leach, 1815, in Brewster. Edinburgh Enc. 9(1), p. 76, 126; syn.: Glossata; ordem.- Leach, 1816, in Brewster. Edinburgh Enc., amer. ed., 8(2), p. 655, 665, 715.Leach, 1819, in Samouelle. Ent. Compend., p. 44, 63, 136, 137, 234.- Hübner, 1816. Verz. bek. Schmett., p. 7; syn.: Glossata.- Billberg, 1820. Enum. Insect. Mus. Billberg, p. 75.- Latreille, 1825. Fam. nat. règne anim., p. 465.Swainson, 1827. Philosoph. Mag. (2)1: 181-187.- Stephens, 1828. Ill. Brit. Ent., Haustellata 1, p. 2.- Latreille, 1829, in Cuvier. Règne Anim. 5, p. 366; syn.: Glossata.- Burmeister, 1829. Insect. syst. nat., p. 10, 12, 15, 16, 27, 28.- Stephens, 1829. Ill. Brit. Ent., Haustellata 2, p. 1.- Leach, 1830, in Brewster. Edinburgh Enc., reed., (4)9, p. 126; syn.: Glossata.Newman, 1834. Ent. Mag. 2: 379, 380.- Boisduval, 1836. Hist. nat. Ins. Sp. gén. Lép. 1, p. 1; syn.: Glossata.Westwood, 1838. Introd. mod. class. ins. 1, p. 24.Westwood, 1840. Introd. mod. class. ins. 2, p. 306; Synopsis gen. brit. ins., p. 87; syn.: Glossata.- Gravenhorst, 1843.

Revista Brasileira de Zoologia 23 (1): 1-41, março 2006 
Vergleichende Zool., p. 165.- Newman, 1870. Entomol. 5: 34.- Burmeister, 1878. Descr. phys. Rép. Arg. 5, p. 1; syn.: Glossata.- Godman \& Salvin, 1879. Biol. Centr.-Amer., Lep.-Rhop. 1, p. 1.- Schoch, 1884. Mitt. Schweiz. ent. Ges. 7: 35, 36.- Brauer, 1885. Sitzb. Ak. Wiss., Wien, 91: 337, 340, 371.- Plötz, 1886. Mitt. naturw. Ver. Neu-Vorpomm. u. Rügen 17: 485.- Haeckel, 1889. Natürl. SchöpfungsGesch., p. 586, 587, 591, 593.- Chapman, 1893. Trans. ent. Soc. London, p. 98, 118.- J. H. Comstock, 1893, in Wilder. The Wilder Quarter-Century Book, p. 96.- Dyar, 1894. Ann. N. Y. Acad. Sc. 8: 201.- Dyar, 1895. Jour. N. Y. Ent. Soc. 3: 17.- Dyar, 1895. Amer. Nat. 29: 1066, 1072.- Packard, 1895. Zool. Anz. 18: 228, 229.- Meyrick, 1895. Handb. brit. Lep., p. 21, 22.- J. H. Comstock \& A. B. Comstock, 1895. Man. Study Ins., p. 204.- Bodine, 1896. Trans. Amer. Ent. Soc. 23: 1; morfologia, antena.- Chapman, 1896. Trans. ent. Soc. London, p. 569, 570, 571.- Tutt, 1897. Ent. Rec. \& Jour. Var. 9: 194, 195, 196.- Holland, 1898. Butt. Book, p. 60, 77.- Börner, 1904. Zool. Anz. 27: 533.- J. H. Comstock \& A. B. Comstock, 1904. Man. Study Ins. $4^{\text {a }}$ ed., p. 191, 196.- Handlirsch, 1906. Die fossilen Ins. 1, p. 44, 618.Handlirsch, 1907. Die fossilen Ins. 2, p. 1215, 1292.- Bode, 1907. Mitt. Roemer-Mus., Hildesheim, 22: 7.- Handlirsch, 1907. Die fossilen Ins. 2, p. 920, 1133, 1253.- Spuler, 1908. Schmett. Europas 1, p. VII.- Stichel, 1911, in Wytsman. Gen. Ins. 1, p. 1.- Heymons, 1915, in zur Strassen. Brehms Tierleben. $4^{\text {a }}$ ed., p. XVIII, 211.- Chapman, 1916. Trans. ent. Soc. London, p. 311.- J. H. Comstock, 1918. Wings Ins., p. 6, 122, 318, 319.- Krausse \& Wolff, 1919. Arch. Natg. 86A(3): 165, 169.- Börner, 1920, in Brohmer. Fauna Deutschland. $2^{\text {a }}$ ed., p. 328.- Fracker, 1920. Ann. Ent. Soc. Amer. 13: 167; biologia.- Forbes, 1923. Mem. Cornell Univ. Agric. Exp. Stat. 68: 62.- Börner, 1925, in Brohmer. Fauna Deutschland. $3^{a}$ ed., p. 358.- Handlirsch, 1925, in Schröder. Handb. Ent. 3, p. 200, 271 359, 383, 393, 852; syn.: Tetraptera alis farinosis, Glossata, Papilionoides, Alata, Gymnoptera, Pterophora, Haustellata, Siphonostoma, Astegoptera, Anelytra, Quadripennia, Tetraptera amorpha adermata, Holometabola, Homoptera, Antliostomata, Insecta haustellata, Ectramoglossata, Micrognatha, Metabola, Ctenoptera, Amplipens, Sugentia, Insecta endoblasta, Euglossata, Cypothoraca, Metagnatha, Sorbentia, Endopterygota, Heterothoraka, Proctanura, Neoptera, Zeugloptera+Lepidoptera [muitos nomes supraordinais, não incluidos aqui].- Meyrick, 1928. Rev. Handb. brit. Lep., p. 29.- Wolff \& Krausse, 1930. Wirbell. Tiere, p. 197.- D'Almeida, 1932. Lambillionea 32(8-9), p. 166.- Börner, 1932, in Brohmer. Fauna Deutschland. $4^{\text {a }}$ ed., p. 369.- D'Almeida, 1937. Mem. Inst. Osw. Cruz 32(2): 246.- Börner, 1939. Verh. VII intern. Kongr. Ent., Berlin, 2, p. 1422.- W. Ph. Comstock, 1944. Sc. Surv. P. Rico and Virgin Isl. 12(4), p. 428.- Turner, 1947. Proc. Linn. Soc. N. S. Wales 71: 306, 337.- Warren, 1947. Entomol. 80: 216.- Hayward, 1948. Gesp. nov. Anim. Arg.
1, p. 5.- Kiriakoff, 1948. Biol. Jaarboek, Antwerpen, 15, p. 120-142; subordem.- Börner, 1949, in Brohmer. Fauna Deutschland. $6^{\text {a }}$ ed., p. 382.- Bourgogne, 1951, in Grassé. Traité Zool. 10(1), p. 174; syn.: Glossata.- Kiriakoff, 1952. Ann. Soc. r. zool. Belg. 83: 91.- Hennig, 1953. Beitr. Ent. 3, Sonderh., p. 47, 76.- Richards \& Davies, 1957, in Imms. Gen. Textb. Ent., p. 511, 533, 534.- Common, 1970, in CSIRO. Ins. Australia, p. 782.- Brock, 1971. Jour. nat. Hist. 5: 29-98.- Razowski, 1974. Acta zool. cracov. 19: 1-15.Heppner, 1984. Atlas neotrop. Lep. 2, Checkl. 1, p. XIX.Common, 1974, in CSIRO. Ins. Australia, Suppl., p. 98.Common, 1975. Ann. Rev. Ent. 20: 183.- Speidel, 1977. Atalanta 8: 119, 120.- Kuznetzov \& Stekolnikov, 1978. Ent. Obozr. 57: 881, 887.- Kuznetzov \& Stekolnikov, 1979. Ent. Rev. 57: 609, 613.- Hennig, 1986. Taschenb. Zool. 3, Wirbellose II. $4^{\text {a }}$ ed., p. 229.- Holloway; Bradley \& Carter, 1987. CIE Guides ins. import. man. 1. Lepidoptera, p. 4, 5.- Nielsen, 1989, in Fernhelm; Bremer \& Jörnvall (Ed.). Hierarchy Life, p. 282, 283.- Scoble, 1989, in Emmet \& Head. Moths and Butt. Great Brit. and Ireland 7, p. 15.Nielsen \& Common, 1991, in CSIRO. Ins. Australia, p. 831, 833, 834.- Scoble, 1992. Lep., p. 12, 188, 190.- Minet, 1991. Ent. scand. 22: 69.- Heppner, 1995. Atlas neotrop. Lep. 3. Checkl. 2, p. XIII.- Heppner, 1996. Atlas neotrop. Lep. 5B. Checkl. 4B, p. XV.- Poole, 1996, in Poole \& Gentili. Nom. Ins. Nearct. 3, p. 612.- Heppner, 1998. Lep. News 1998 (3): 57.- Heppner, 1998. Holarct. Lep. 5, Suppl 1: 1, 2, 3, 7, 18.- Kristensen \& Skalski, 1999, in Kristensen (Ed.), Lep. 1, p. 9, 15, in M. Fischer (Ed.). Handb. Zool. 4.- Heppner, 2003. Lep. Florida 1, p. 71, 91, 94, 231.

Noctuae Linnaeus, 1758. Syst. nat. $10^{\mathrm{a}}$ ed, p. 508; status de gênero.- Guenée, 1875. Stat. sc. d'Eure-et-Loir. Lép., p. 164; legião, incluindo Trifidae, Quadrifidae.- Chapman, 1893. Trans. ent. Soc. London, p. 104; sem status, sem indicação de grupo.

Tetraptera alis farinaceis Geoffroy, 1762. Hist. abregée Ins. 2, p. 25; sem status [= Lepidoptera].

Tetrapi Scopoli, 1763. Ent. Carniolica, p. 142; [divisão] de Papilio, oposta à Hexapi, incluindo os [Nymphalidae].Guenée, 1875. Stat. sc. d'Eure-et-Loir. Lép., p. 17; falange, incluindo Spinosae, Carunculatae, Furculae.

Hexapi Scopoli, 1763. Ent. Carniolica, p. 166; [divisão] de Papilio, oposta à Tetrapi, incluindo as demais diurnas.Guenée, 1875. Stat. sc. d'Eure-et-Loir. Lép., p. 10; falange, incluindo Tentaculatae, Granulosae.

Bombyces Scopoli, 1763. Ent. Carniolica, p. 191; sem status, incluindo espécies.

Geometrae Scopoli, 1763. Ent. Carniolica, p. 214; sem status, incluindo espécies.- Latreille, 1817, in Cuvier. Règne Anim. 3, p. 570; tribo, incluindo [Geometridae].- Guenée, 1875. Stat. sc. d'Eure-et-Loir. Lép., p. 91.

Tortrices Scopoli, 1763. Ent. Carniolica, p. 232; sem status, in- 
cluindo espécies.- Latreille, 1817, in Cuvier. Règne Anim. 3, p. 576; tribo, incluindo [Tortricidae].- Latreille, 1825. Fam. nat. règne anim., p. 475.- Chapman, 1893. Trans. ent. Soc. London, p. 102, 107, 111, 113, 114.

Pyrales Scopoli, 1763. Ent. Carniolica, p. 239; sem status, incluindo espécies.- Latreille, 1817, in Cuvier. Règne Anim. 3, p. 577; tribo, incluindo [Pyralidae].

Tineae Scopoli, 1763. Ent. Carniolica, p. 243; sem status, incluindo espécies.- Latreille, 1817, in Cuvier. Règne Anim. 3, p. 577; tribo incluindo [Tineidae]..

Alucitae Scopoli, 1763. Ent. Carniolica, p. 256; sem status, incluindo espécies.

Farinacea Schluga, 1767. Prim. lin. cogn. Insect., p. 21; ordem [= Lepidoptera $]$.

Farinosa Schluga, 1767. Prim. lin. cogn. Insect., p. 21, 36; ordem [= Lepidoptera].

Glossata Fabricius, 1775. Syst. ent., p. 9, 442 [= Lepidoptera].Fabricius, 1776. Gen. ins., p. 155.- Fabricius, 1781. Spec. ins. 2, p. 1.- Jablonsky, 1783. Naturs. bek. in- und ausländ. Ins., p. 184.- Fabricius, 1787. Mant. ins. 2, p. 1.- Roemer, 1789. Gen. ins. Linn. Fabr., p. 67.- Fabricius, 1793. Ent. syst. 3(1), p. [III].- Leach, 1815, in Brewster. Edinburgh Enc. 9(1), p. 72.- Leach, 1816, in Brewster. Edinburgh Enc., amer ed., 8(2), p. 661.- Leach, 1819, in Samouelle. Ent. Comp., p. 234.- Westwood, 1838. Introd. mod. class. ins. 1, p. 22; syn.: Lepidoptera.- Burmeister, 1839. Handb. Ent. 2, p. 39, 41; syn.: Lepidoptera.- Packard, 1895. Amer. Nat. 29: 636, 637, 643; syn.: Haustellata; subordem.- Karsch, 1898. Ent. Nachr. 24: 297.- Börner, 1920, in Brohmer. Fauna Deutschland. $2^{\text {a }}$ ed., p. 341; syn.: Eulepidoptera, Haustellata, Frenatae.- Fabricius, 1938. Syst. glossat., p. III.- Börner, 1939. Verh. VII intern. Kongr. Ent., Berlin, 2, p. 1372, 1389.Kiriakoff, 1946. Lambillionea 46: 112.- Hennig, 1953. Beitr. Ent. 3, Sonderh., p. 47.- Kiriakoff, 1955. Bull. \& Ann. Soc. ent. Belg. 91: 155, 158.- Razowski, 1974. Acta zool. cracov. 19: 10.- Speidel, 1977. Atalanta 8: 119, 120.- Küppers \& Speidel, 1980. Atalanta 11: 58, 59, 63; syn.: Haustellata.Kristensen \& Nielsen, 1981, in Cederholm. Ent. scand., Suppl. 15: 300, 303.- Kristensen \& Nielsen, 1983. Ztschr. zool. Syst. Evolut.-forsch. 21: 114, 115, 116, 118, 119.Kristensen, 1984. Steenstrupia 10: 160, 170, 186.- Heppner, 1984. Atlas neotrop. Lep. 2. Checkl. 1, p. XIX.- Minet, 1984. Nouv. Rev. Ent. (n. s.) 1: 147.- Kristensen, 1985. Ent. Medd. 52: 9.- Nielsen, 1985. Jour. Res. Lep., Suppl. 1: 3, 15.- Minet, 1986. Alexanor 14: 291, 292.- Hennig, 1986. Taschenb. Zool. 3, Wirbellose II. $4^{\text {a }}$ ed., p. 231.- Holloway; Bradley \& Carter, 1987. CIE Guides ins. import. man. 1. Lepidoptera, p. 18, 23, 25, 38.- Nielsen, 1989, in Fernholm; Bremer \& Jörnvall (Eds). Hierarchy Life, p. 281, 282, 284, 285, 291.- Scobel, 1989, in Emmet \& Head. Moths and Butt. Great Brit. and Ireland 7, p. 15, 17, 21.- Nielsen \& Common, 1991, in CSIRO. Ins. Australia, p. 832, 833, 834, 837.- Minet, 1991. Ent. scand. 22: 69.- Scoble, 1992. Lep., p. 13, 188, 195, 199.- Minet, 1994. Ent. scand. 25: 63.Heppner, 1995. Atlas neotrop. Lep. 3. Checkl. 2, p. XIII.Poole, 1996, in Poole \& Gentili. Nom. Ins. Nearct. 3, p. 612.- Heppner, 1996. Atlas neotrop. Lep. 5B. Checkl. 4B, p. XV.- Nielsen \& Kristensen, 1996. Invert. Tax. 10: 1200, $1201,1251,1253-1255,1258,1259,1261-1263,1265,1274-$ 1276, 1291-1293.- Heppner, 1998. Lep. News 1998 (3): 57.Heppner, 1998. Holarct. Lep. 5, Suppl. 1: 7, 18.- Kristensen \& Skalski, 1999, in Kristensen (Ed.), Lep. 1, p. 10, 11, in Fischer (Ed.). Handb. Zool. 4.- Carter \& Kristensen, 1999, in Kristensen (Ed.), Lep. 1, p. 28, in Fischer (Ed.). Handb. Zool. 4.- Kristensen, 1999, in Kristensen (Ed.), Lep. 1, p. 43, in Fischer (Ed.). Handb. Zool. 4.- Kristensen, 1999, in Kristensen (Ed.), Lep. 1, p. 51, in Fischer (Ed.). Handb. Zool. 4.- Powell et al., 1999, in Kristensen (Ed.), Lep. 1, p. 406, in Fischer (Ed.). Handb. Zool. 4.- Heppner, 2003. Lep. Florida 1, p. 71, 91, 94, 231; subordem.

Papilionoides Laicharting, 1781. Verz. Beschr. Tyroler-Ins. 1(1), p. IV; syn.: Lepidoptera, Tetraptera alis farinaceis, Glossata; ordem.

Lépidoptères [sic]; Latreille, 1796. Précis caract., p. 140.Latreille, 1803. Hist. nat., gén. part. Crust. Ins. 5, p. 319, 325.- Duméril, 1806. Zool. analyt. méth. nat. class. anim., p. XXXI, 271.- Latreille, 1817, in Cuvier. Règne Anim. 3, p. 535; syn.: Glossata.- Duméril, 1823. Cons. gén., p. 89, 134, 139, 163, 222.- Latreille, 1825. Fam. nat. règne anim., p. 465.- Blanchard, 1850, in Castelnau. Hist. nat. Anim. art. 3(2), p. 417.- Duméril, 1860. Ent. analyt. 2, p. 1088, 1089.Lameere, 1931. Rec. Inst. zool. Torley-Rousseau 3(2): 24, 234.

Lepidioptera [sic]; Clairville, 1798. Entom. helv. 1, p. 44, 45.

Haustellata Clairville, 1798. Entom. helv. 1, p. 44, 45; part., supraordinal, incluindo Halteriptera [= Diptera], Lepidioptera, Hemimeroptera $[=$ Heteroptera].- Leach, 1819, in Samouelle. Ent. Compend., p. 136; part., supraordinal.- Stephens, 1828. Ill. Brit. Ent., Haustellata 1, p. 1 (part., supraordinal).- Packard, 1895. Zool. Anz. 18: 229, 233; syn.: Glossata; subordem, incluindo Palaeolepidoptera, Neolepidoptera.- Packard, 1895. Amer. Nat. 29: 643.- Packard, 1895. Mem. Nat. Acad. Sc. 7: 61 (part.); syn.: Glossata.Tutt, 1897. Ent. Rec. \& Jour. Var. 9: 196; syn.: Palaeolepidoptera, Neolepidoptera.- Karsch, 1898. Ent. Nachr. 24: 297.- Tutt, 1899. Brit. Lep. 1, p. 106, 114.- Kiriakoff, 1946. Lambillionea 46: 24, 112; syn.: Neolepidoptera, Palaeolepidoptera.- Friese, 1969. Bericht 10. Wandervers. Deutscher Ent., Tagungsb. 80(2): 205, 207, 218, 219.Razowski, 1974. Acta zool. cracov. 19: 9.- Kuznetzov \& Stekolnikov, 1978. Ent. Obozr. 57: 881, 883, 887.Kuznetzov \& Stekolnikov, 1979. Ent. Rev. 57: 609, 610, 613. Diurnes [sic]; Latreille, 1803. Hist. nat., gén part. Crust. Ins. 5, p. 319, 320.- Blanchard, 1850, in Castelnau. Hist. nat. Anim. art. 3(2), p. 419; syn.: Rhopalocères.

Nocturnes [sic]; Latreille, 1803. Hist. nat., gén. part. Crust. Ins. 
5, p. 321.- Latreille, 1829, in Cuvier. Règne Anim. 5, p. 395.- Blanchard, 1850, in Castelnau. Hist. nat. Anim. art. 3(2), p. 481; inclue parte das mariposas.

Diurni Latreille, 1805. Hist. nat., gén. part. Crust. Ins. 14, p. 76; seção, incluindo as diurnas [e parte das noturnas].- Fabricius, 1807. Mag. Insektenk. 6: 290.- Boisduval \& Leconte, 1829. Hist. gén. icon. Lép. Chen. l'Amer. sept., p. 1.- Lucas, 1864. Hist. nat. Lép. exot., p. 4.- Newman, 1870. Entomol. 5: 34. Guenée, 1875. Stat. sc. d'Eure-et-Loir. Lép., p. 9; sem status, incluindo Bicalcarati e Quadricalcarati.- Scoble, 1992. Lep., p. 190.

Papilionides Latreille, 1805. Hist. nat., gén. part. Crust. Ins. 14, p. 76; família, incluindo [Papilionoidea e Hesperioidea].- Leach, 1815, in Brewster. Edinburgh Enc. 9(1), p. 126; tribo, incluindo as famílias Papilionida, Lycaenida.- Leach, 1816, in Brewster. Edinburgh Enc., amer. ed., 8(2), p. 715; tribo, incluindo as famílias Papilionida, Lycaenida.- Latreille, 1825. Fam. nat. règne anim., p. 467; tribo, incluindo [Papilionoidea].Leach, 1830, in Brewster. Edinburgh Enc., reed., (4)9, p. 126; tribo, incluindo Papilionida, Lycaenida.- Grote, 1895. Mitt. Roemer-Mus., Hildesheim, 1: [2]; superfamília.- Grote, 1897. Mitt. Roemer-Mus., Hildesheim, 8: 7.

Nocturni Latreille, 1805. Hist. nat., gén. part. Crust. Ins. 14, p. 142; seção, incluindo Bombycinae.- Newman, 1870. Entomol. 5: 34.- Guenée, 1875. Stat. sc. d'Eure-et-Loir. Lép., p. 41; sem status, incluindo Areolati, Quadrifidae, Radiati.- Scoble, 1992. Lep., p. 190.

Glossates [sic]; Duméril, 1806. Zool. analyt. méth. nat. class. anim., p. 270.

Ropalocères [= Rhopalocera] Duméril, 1806. Zool. analyt. méth. nat. class. anim., p. XXXI, 270, 271, pl. 271; syn.: Globulicornes; família, incluindo Papillon, Hétéroptères, Hespérie - genres.- Duméril, 1823. Cons. gén., p. 139, 163; syn.: Globulicornes.- Duméril, 1860. Ent. analyt. 2, p. 1089, 1090, 1091, 1092, 1099, 1101.- Lameere, 1931. Rec. Inst. zool. Torley-Rousseau 3(2): 235.- Lameere, 1938. Rec. Inst. zool. Torley-Rousseau 5: 170-179.

Chétocères Duméril, 1806. Zool. analyt. méth. nat. class. anim., p. XXXI, 271, 275; syn.: Séticornes; família, incluindo Ptérophore, Phalène, Crambe, Alucite, Noctuelle, Pyrale, Teigne, Lithosie - genres.- Duméril, 1823. Cons. gén., p. 139, 163, 224; syn.: Séticornes; família, incluindo Phalène, Pterophore, Lithosies, Teignes, Pyrales, Alucites, Crambes, Noctuelles.Duméril, 1823. Dict. sc. nat. 26, p. 43; syn.: Séticornes [= Heterocera "micros"].- Duméril, 1860. Ent. analyt. 2, p. 1089, 1091, 1146, 1149; syn.: Séticornes.

Séticornes Duméril, 1806. Zool. analyt. méth. nat. class. anim., p. 275; na sinonímia de Chétocères.- Duméril, 1823. Cons. gén., p. 224; syn.: Chétocères; família, incluindo Phalène, Pterophore, Lithosies, Teignes, Pyrales, Alucites, Crambes, Noctuelles.- Latreille, 1825. Fam. nat. règne anim., p. 471; syn.: Nocturnes.
Globulicornes Duméril, 1806. Zool. analyt. méth. nat. class. anim., p. 271; na sinonímia de Ropalocères.- Duméril, 1823. Cons. gén., p. 222; syn.: Ropalocères; família, incluindo as diurnas.- Duméril, 1823. Dict. sc. nat. 26, p. 43; nome na sinonímia de Rhopalocères.- Boisduval \& Leconte, 1829. Hist. gén. icon. Lép. Chen. l'Amer. sept., p. 1.

Clostérocères Duméril, 1806. Zool. analyt. méth. nat. class. anim., p. XXXI, 271, 273; syn.: Fusicornes; família, incluindo Sésie, Sphinx, Zygene - genres.- Duméril, 1823. Cons. gén., p. 139, 163; syn.: Fusicornes; família, incluindo Sphinx, Sésie, Zygènes.- Duméril, 1823. Dict. sc. nat. 26, p. 43, 44; syn.: Fusicornes [= Heterocera "macros"].- Rambur, 1842. Faune ent. de L'Andalousie. $2^{a}$ ed., p. 322; divisão; syn.: Sphinx Linné, Crépusculaires, Hétérocères.- Duméril, 1860. Ent. analyt. 2, p. 1089, 1090, 1105, 1109; syn.: Fusicornes.

Fusicornes Duméril, 1806. Zool. analyt. méth. nat. class. anim., p. 273; na sinonímia de Clostérocères.- Duméril, 1823. Cons. gén., p. 222; syn.: Clostérocères; família, incluindo Sphinx, Sésies, Zygènes.- Latreille, 1825. Fam. nat. règne anim., p. 471; syn.: Crepusculaires.

Nématocères Duméril, 1806. Zool. analyt. méth. nat. class. anim., p. XXXI, 271, 273; syn.: Filicornes; família, incluindo Hépiale, Bombyx, Cossus - genres.- Duméril, 1823. Cons. gén., p. 139, 163; syn.: Filicornes; família, incluindo Bombyces, Cossus, Hepialus.- Duméril, 1823. Dict. sc. nat. 26, p. 43, 44; syn.: Filicornes [= Heterocera].- Duméril, 1860. Ent. analyt. 2, p. 1089, 1091, 1125, 1127, 1131; syn.: Filicornes.

Filicornes Duméril, 1806. Zool. analyt. méth. nat. class. anim., p. 273; na sinonímia de Nématocères.- Duméril, 1823. Cons. gén., p. 223; syn.: Nématocères; família, incluindo Bombyces, Cossus, Hepialus.

Diurna [sic]; Latreille, 1809. Gen. Crust. Ins. 4, p. 186, 193; tribo, incluindo Papilionides, Hesperides.- Leach, 1815, in Brewster. Edinburgh Enc. 9(1), p. 126; seção, incluindo as tribos Papilionides e Hesperides.- Leach, 1816, in Brewster. Edinburgh Enc., amer. ed., 8(2), p. 715; seção.- Latreille, 1817, in Cuvier. Règne Anim. 3, p. 543; família, incluindo as diurnas.- Leach, 1819, in Samoulle. Ent. Compend., p. 234; seção.- Latreille, 1825. Fam. nat. règne anim., p. 466 [= Papilionoidea].- Swainson, 1827. Philosoph. Mag. (2)1: 182.- Stephens, 1828. Ill. Brit. Ent., Haustellata 1, p. 4, 96.- Latreille, 1829, in Cuvier. Règne Anim. 5, p. 374.Leach, 1830, in Brewster. Edinburgh Enc., reed., (4)9, p. 126.- Zetterstedt, 1839. Ins. Lapponica, p. 885.- Westwood, 1840. Introd. mod. class. ins. 2, p. 325.- Gravenhorst, 1843. Vergleichende Zool., p. 168.- Duponchel, 1844. Cat. méth. Lep. d'Europe, p. 1; syn.: Ropalocères, Rhopalocera.Burmeister, 1878. Descr. phys. Rép. Arg. 5, p. 44.- Jordan, 1907, in Seitz. Gross-Schmett. Erde 5, p. 11.- Spuler, 1908. Schmett. Europas 1, p. XX [= Rhopalocera], p. 1 [divide em Rhopalócera e Netrócera].

Revista Brasileira de Zoologia 23 (1): 1-41, março 2006 
Crepuscularia Latreille, 1809. Gen. Crust. Ins. 4, p. 187, 209; tribo, incluindo Sphingides, Zygénides.- Leach, 1815, in Brewster. Edinburgh Enc. 9(1), p. 130; seção, incluindo as tribos Sphingides, Zygaenides.- Leach, 1816, in Brewster. Edinburgh Enc., amer. ed., 8(2), p. 719; seção.- Latreille, 1817, in Cuvier. Règne Anim. 3, p. 555.- Leach, 1819, in Samouelle. Ent. Comp., p. 243.- Latreille, 1825. Fam. nat. règne anim., p. 470.- Stephens, 1828. Ill. Brit. Ent., Haustellata 4, p. 104.- Latreille, 1829, in Cuvier. Règne Anim. 5, p. 387.- Leach, 1830, in Brewster. Edinburgh Enc., reed., (4)9, p. 130.- Zetterstedt, 1839. Ins. Lapponica, p. 885; syn.: Scotina, Heterocera.- Westwood, 1840. Introd. mod. class. ins. 2, p. 325, 361, 363.- Gravenhorst, 1843. Vergleichende Zool., p. 168.- Burmeister, 1878. Descr. phys. Rép. Arg. 5, p. 45, 307.- Duponchel, 1844. Cat. méth. Lép. d'Europe, p. 39; syn.: Clostérocères, Héterocères.

Nocturna [sic]; Latreille, 1809. Gen. Crust. Ins. 4, p. 189, 216; tribo, incluindo Bombycites, Noctuo-Bombycites, Noctuaelites, Phalenites, Pyralites, Pterophorites.- Leach, 1815, in Brewster. Edinburgh Enc. 9(1), p. 131; seção, incluindo as tribos Bombycides, Arctiides, Tineides, Noctuides, Phalaenides, Pyralides, Alucitides.- Leach, 1816, in Brewster. Edinburgh Enc., amer. ed., 8(2), p. 720; seção.- Latreille, 1817, in Cuvier. Règne Anim. 3, p. 555; família.- Leach, 1819, in Samouelle. Ent. Comp., p. 245.- Latreille, 1825. Fam. nat. règne anim., p. 471.- Stephens, 1829. Ill. Brit. Ent., Haustellata 2, p. 1.Leach, 1830, in Brewster. Edingburg Enc., reed., (4)9, p. 131.- Westwood, 1840. Introd. mod. class. ins. 2, p. 325, 361, 363.- Duponchel, 1844. Cat. méth. Lép. d’Europe, p. 54; syn.: Nématocères, Chetocères, Héterocères.- Burmeister, 1878. Descr. phys. Rép. Arg. 5, p. 44, 362.

Bombycites Latreille, 1809. Gen. Crust. Ins. 4, p. 190; família.Latreille, 1810. Cons. gén., p. 441.- Latreille, 1817, in Cuvier. Règne Anim. 3, p. 561; tribo, incluindo [Saturniidae, Hepialidae, Cossidae].- Latreille, 1825. Fam. nat. règne anim., p. 472.- Latreille, 1829, in Cuvier. Règne Anim. 5, p. 399.

Noctuo-Bombycites Latreille, 1809. Gen. Crust. Ins. 4, p 190; família.- Latreille, 1810. Cons. gén., p. 441.- Latreille, 1817, in Cuvier. Règne Anim. 3, p. 569; tribo, incluindo [Arctiidae].

Noctuaelites Latreille, 1809. Gen. Crust. Ins. 4, p. 191; família.- Latreille, 1810. Cons. gén., p. 441.- Latreille, 1817, in Cuvier. Règne Anim. 3, p. 573; tribo, incluindo [Noctuidae].Latreille, 1825. Fam. nat. règne anim., p. 475.- Latreille, 1829, in Cuvier. Règne Anim. 5, p. 408.

Phalenites Latreille, 1809. Gen. Crust. Ins. 4, p. 191; família.Latreille, 1810. Cons. gén., p. 441.

Pterophorites Latreille, 1809. Gen. Crust. Ins. 4, p. 192; família.Latreille, 1810. Cons. gén., p. 441.- Latreille, 1817, in Cuvier. Règne Anim. 3, p. 583; tribo, incluindo [Pterophoridae].Latreille, 1825. Fam. nat. règne anim., p. 478.

Pyralites Latreille, 1809. Gen. Crust. Ins. 4, p. 192; família.Latreille, 1810. Cons. gén., p. 441.- Guenée, 1854, in Boisduval \& Guenée. Hist. nat. Ins. Sp. gén. Lép. 8, p. 97; syn.: Pyralis L.; divisão, incluindo as tribos Sqamosae, Pulverulentae, Luridae, Plicatae.- Stainton, 1859. Man. Brit. Butt. and Moths 2, p. 124; seção, incluindo Squamosae [sic], Pulverulentae, Luridae.

Crambites Latreille, 1810. Cons. gén., p. 441.- Latreille, 1825. Fam. nat. règne anim., p. 478; tribo, incluindo [Crambidae].

Tineites Latreille, 1810. Cons. gén., p. 441; família.- Latreille, 1817, in Cuvier. Règne Anim. 3, p. 577; syn.: Phalaenae Tineae Linnaeus; tribo, incluindo [Tineidae].- Latreille, 1825. Fam. nat. règne anim., p. 474; tribo, incluindo [Lithosiidae, Yponomeutidae, Oecophoridae, Adelidae, Tineidae].- Latreille, 1829, in Cuvier. Règne Anim. 5, p. 415.

Lepidopteria [sic]; Rafinesque, 1815. Analyse Nat., p. 127.

Diurnaria [sic]; Rafinesque, 1815. Analyse Nat., p. 127; subordem, incluindo [Papilionoidea e Hesperoidea].

Ropaloceria [sic]; Rafinesque, 1815. Analyse Nat., p. 127; família, incluindo parte das diurnas, não [Hesperioidea].

Nocturnaria [sic]; Rafinesque, 1815. Analyse Nat., p. 128; subordem, incluindo parte de mariposas.

Closteroceria [sic]; Rafinesque, 1815. Analyse Nat., p. 128; família, incluindo [Sphingidae e Zygaenidae].

Platypteria Rafinesque, 1815. Analyse Nat., p. 128; família, incluindo parte de mariposas.

Erephoteria Rafinesque, 1815. Analyse Nat., p. 129; família, incluindo parte de mariposas.

Glossostoma Leach, 1815, in Brewster. Edinburgh Enc. 9(1), p. 76; cohors, incluindo só a ordem Lepidoptera.- Leach, 1816, in Brewster. Edinburgh Enc., amer. ed., 8(2), p. 665; cohors, incluindo só a ordem Lepidoptera.- Leach, 1830, in Brewster. Edinburgh Enc., reed., (4)9, p. 76; cohors, incluindo só a ordem Lepidoptera.

Pyralides Leach, 1815, in Brewster. Edinburgh Enc. 9(1), p. 135; tribo, incluindo as famílias Pyralida, Tineida.- Leach, 1816, in Brewster. Edinburgh Enc., amer. ed., 8(2), p. 724; tribo, incluindo Pyralida, Tineida.- Latreille, 1817, in Cuvier. Règne Anim. 3, p. 572; tribo, incluindo [Pyralidae].- Leach, 1830, in Brewster. Edinburgh Enc., reed., (4)9, p. 135.

Sphingides Leach, 1815, in Brewster. Edinburgh Enc. 9(1), p. 130; tribo, incluindo espécies de [Sphingidae].- Leach, 1816, in Brewster. Edinburgh Enc., amer. ed., 8(2), p. 719; tribo, incluindo espécies de [Sphingidae].- Latreille, 1825. Fam. nat. règne anim., p. 471; tribo de Crepuscularia, incluindo [Sphingidae].- Leach, 1830, in Brewster. Edinburgh Enc., reed., (4)9, p. 130.

Zygaenides Leach, 1815, in Brewster. Edinburgh Enc. 9(1), p. 131; tribo sem famílias, incluindo espécies de [Sesiidae, Zygaenidae].- Leach, 1816, in Brewster. Edinburgh Enc., amer. ed., 8(2), p. 720; tribo, incluindo espécies de [Sesiidae, Zygaenidae].- Latreille, 1825. Fam. nat. règne anim., p. 471; tribo, incluindo [Sesiidae, Thyrididae, Zygaenidae, Ctenuchidae].Leach, 1830, in Brewster. Edinburgh Enc., reed., (4)9, p. 131. Bombycides Leach, 1815, in Brewster. Edinburgh Enc. 9(1), p.

Revista Brasileira de Zoologia 23 (1): 1-41, março 2006 
131; tribo, incluindo as famílias Cossida, Bombycida.- Leach, 1816, in Brewster. Edinburgh Enc., amer. ed., 8(2), p. 720; tribo, incluindo as famílias Cossida, Bombycida.- Leach, 1830, in Brewster. Edinburgh Enc., reed., (4)9, p. 131.

Arctides Leach, 1815, in Brewster. Edinburgh Enc. 9(1), p. 133; syn.: Noctuo-Bombycites Latreille; tribo sem famílias, incluindo espécies de [Arctiidae].- Leach, 1816, in Brewster. Edinburgh Enc., amer. ed., 8(2), p. 722; syn.: NoctuoBombycites Latreille; tribo, incluindo espécies de [Arctiidae].Leach, 1830, in Brewster. Edinburgh Enc., reed., (4)9, p. 133; syn.: Noctuo-Bombycites Latreille.

Tineides Leach, 1815, in Brewster. Edinburgh Enc. 9(1), p. 133; tribo, incluindo as famílias Tineida, Nemophorida.- Leach, 1816, in Brewster. Edinburgh Enc., amer. ed., 8(2), p. 722; tribo, incluindo as famílias Tineida, Nemophorida.- Leach, 1830, in Brewster. Edinburgh Enc., reed., (4)9, p. 133.- Le Marchand, 1945. Rev. franç. Lép. 10: 104; grupo de Tineina, sem incluir taxa.

Noctuides Leach, 1815, in Brewster. Edinburgh Enc. 9(1), p. 133; tribo, incluindo as famílias Erebida, Noctuida.- Leach, 1816, in Brewster. Edinburgh Enc., amer. ed., 8(2), p. 722; tribo, incluindo as famílias Erebida, Noctuida.- Leach, 1830, in Brewster. Edinburgh Enc., reed., (4)9, p. 133.

Phalaenides Leach, 1815, in Brewster. Edinburgh Enc. 9(1), p. 134; tribo, incluindo as famílias Phalaenida, Geometrina, Herminida, Platyptericida, Tortricidae.- Leach, 1816, in Brewster. Edinburgh Enc., amer. ed., 8(2), p. 723; tribo, incluindo as famílias Phalaenida, Geometrida, Herminida, Platyptericida, Tortricida.- Leach, 1830, in Brewster. Edinburgh Enc., reed., (4)9, p. 134.

Alucitides Leach, 1815, in Brewster. Edinburgh Enc. 9(1), p. 135; syn.: Pterophorites Latreille; tribo sem famílias, incluindo espécies de [Pterophoridae].- Leach, 1816, in Brewster. Edinburgh Enc., amer. ed., 8(2), p. 724; syn.: Pterophorites; tribo, incluindo [Pterophoridae].- Leach, 1830, in Brewster. Edinburgh Enc., reed., (4)9, p. 135; syn.: Pterophorites Latreille.

Deltoides Latreille, 1817, in Cuvier. Règne Anim. 3, p. 372; syn.: Phalaenae Pyralides; tribo, incluindo o subgênero Botys.Latreille, 1829, in Cuvier. Règne Anim. 5, p. 415.- Guenée, 1854, in Boisduval \& Guenée. Hist. nat. Ins. Sp. gén. Lép. 8, p. 1; divisão, incluindo Platydidae, Hypenidae, Herminidae.Stainton, 1859. Man. Brit. Butt. and Moths 2, p. 124, 125; seção, incluindo Hypenidae, Herminidae.

Arpenteuses Latreille, 1817, in Cuvier. Règne Anim. 3, p. 570; syn.: Phalaenae, Geometrae, Phalaenites; tribo, incluindo Phalaena.- Latreille, 1829, in Cuvier. Règne Anim. 5, p. 413.

Tordeuses Latreille, 1817, in Cuvier. Règne Anim. 3, p. 576; syn.: Phalaenae Tortrices; tribo, incluindo [Tortricidae].

Alucites Latreille, 1817, in Cuvier. Règne Anim. 3, p. 580; syn.: Alucita Fabricius, Adèles Latreille; tribo, incluindo [Alucitidae].

Teignes Latreille, 1817, in Cuvier. Règne Anim. 3, p. 580; syn.: Tinea; tribo, incluindo [Tineidae].
Fissipennes Latreille, 1817, in Cuvier. Règne Anim. 3, p. 583; syn.: Pterophorites; tribo, incluindo Pterophorus.- Latreille, 1829, in Cuvier. Règne Anim. 5, p 423.

Ephemerina Billberg, 1820. Enum. Insect. Mus. Billberg, p. 75; tribo, incluindo os Lepidoptera diurnos.

Scotina Billberg, 1820. Enum. Insect. Mus. Billberg, p. 81; tribo, incluindo os Lepidoptera noturnas.

Rhopalocera; Duméril, 1823. Cons. gén., p. 123.- Boisduval, 1840. Gen. Ind. meth. Europ. Lep., p. 1; legião.- Westwood, 1840. Introd. mod. class. ins. 2, p. 329, 330, 345; Synopsis gen. brit. Ins., p. 87; syn.: Diurna; seção.- Duponchel, 1844. Cat. méth. Lép. d'Europe, p. 1.- Lederer, 1853. Verh. zool.bot. Ges. Wien 25: 14, 17.- Walker, 1854. List Specimens Lep. Ins. Coll. Brit. Mus. 1, p. 1.- Herrich-Schäffer, 1856, [1858]. Samml. aussereurop. Schmett. 1, p. 2 (1856), p. 53 ([1858]).- Stainton, 1857. Man. Brit. Butt. and Moths 1, p. 10.- Bates, 1862. Trans. Linn. Soc. London 23: 515.Newman, 1870. Entomol. 5: 34.- Burmeister, 1878. Descr. phys. Rép. Arg. 5, p. 44, 46.- Godman \& Salvin, 1879. Biol. Centr.-Amer., Lep.-Rhop. 1, p. 1.- Snellen, 1882. Tijdschr. Ent. 25: 215.- Plötz, 1886. Mitt. naturw. Ver. NeuVorpomm. u. Rügen 17: 485, 488.- Haase, 1891. Dtsch. ent. Ztschr. "Iris" 4: 2, 3, 25, 26, 27, 32.- Dyar, 1895. Jour. N. Y. Ent. Soc. 3: 26.- Dyar, 1895. Trans. N. Y. Ac. Sc. 14: 52, 53, 60, 62.- Chapman, 1895. Ent. Rec. \& Jour. Var. 6: 101.- Dyar, 1895. Ent. News 6: 39; superfamília.- Reuter, 1896. Acta Soc. Sc. Fenn. 22(1): I-XI, 192, 200, 201, 203 $207,211,213,214,541-548,550,555,557,558$, pl. 6 (filogenia).- Chapman, 1896. Trans. ent. Soc. London, p. 578.- Tutt, 1896. Brit. Butt., p. 85, 116.- Reuter, 1898. Ent. Rec. \& Jour. Var. 10: 97; subordem.- Karsch, 1898. Ent. Nachr. 24: 297, 298, 300, 301.- Holland, 1898. Butt. Book, p. $60,77 .-$ Tutt, 1899. Brit. Lep. 1, p. 107.- Kirby, [1900], in Hübner. Samml. exot. Schmett. 3, reed., p. 1.- Jordan, 1907, in Seitz. Gross-Schmett. Erde 5, p. 11, 383.- Raymundo da Silva, 1907. Lep. Brasil, p. 5; syn. Achalinoptera.- Handlirsch, 1907. Die fossilen Ins. 2, p. 1134, 1258.- Kirby, 1908-1912, in Hübner. Zutr. Samml. Exot. Schmett., reed., p. 1.- Spuler, 1908. Schmett. Europas 1, p. 1.- Heymons, 1915, in zur Srassen. Brehms Tierleben. $4^{\mathrm{a}}$ ed., p. XXI, 288.- Fracker, 1915. Illinois Biol. Monogr. 2: 119.- Börner, 1920, in Brohmer. Fauna Deutschland. $2^{a}$ ed., p. 354; syn.: Diurna.Lindsey, 1921. Univ. Iowa Stud. Nat. Hist. 9(4): 7.- Forbes, 1923. Mem. Cornell Univ. Agric. Exp. Stat. 68: 43.Handlirsch, 1925, in Schröder. Handb. Ent. 3, p. 273, 934; syn.: Diurna, Papiliones, Diurni, Papilionides, Rhopaloceres, Papilionidae, Ephemerina, Papillonides, Papilionacea, Papilionina, Papilionites, Lep. pedunculata, Detegentes, Celantes, Papilionoidea, Lep. Harmoncopoda, Enantioneura.- H. von Ihering, 1929. Ent. Rdsch., Stuttgart, 46: 29-30, 33-34, 4142, sep., p. 6 [divide os Rhopalocera em Holopoda e Peropoda].D'Almeida, 1932. Lambillionea 32(8-9): 169.- D'Almeida, 1937. Mem. Inst. Osw. Cruz 32(2): 246.- Puységur, 1938. 
Riv. Sci., Nice, 25: 9, 13, syn.: Diurna, Diurni, Achalinoptera, Papilionina.- Börner, 1939. Verh. VII intern. Kongr. Ent., Berlin, 2, p. 1403, 1407, 1422.- W. Ph. Comstock, 1944. Sc. Surv. P. Rico and Virgin Isl. 12(4), p. 428; syn.: Ropalocères.Holland, 1945. Butt. Book, p. 63.- Kiriakoff, 1946. Lambillionea 46: 112.- Agenjo, 1946. Graellsia 4(3): [8].Turner, 1947. Proc. Linn. Soc. N. S. Wales 71: 303, 310337.- Hayward, 1948. Gesp. nov. Anim. Arg. 1, p. 8.Kiriakoff, 1948. Biol. Jaarboek, Antwerpen, 15, p. 140, 141.Börner, 1949, in Brohmer. Fauna Deutschland. $6^{\mathrm{a}}$ ed., p. 416.- Bourgogne, 1951, in Grassé. Traité Zool. 10(1), p. 426.Hennig, 1953. Beitr. Ent. 3, Sonderh., p. 47.- Richards \& Davies, 1957, in Imms. Gen. Textb. Ent., p. 533.- Forbes, 1960. Mem. Cornell Univ. Agric. Exp. Stat. 371: 58.- Friese, 1969. Bericht 10. Wandervers. Deutscher Ent., Tagungsb. 80(2): 205, 207.- Razowski, 1974. Acta zool. cracov. 19: 8, 9.- Common, 1975. Ann. Rev. Ent. 20: 183.- Gómez B., 1978. Shilap, Revta lepid. 6: 249.- Bridges, 1984. Lep.: Rhop. Notes Fam.-Group Nam. 2, p. 1.- Hennig, 1986. Taschenb. Zool. 3, Wirbellose II. $4^{\text {a }}$ ed., p. 232, 234.Holloway; Bradley \& Carter, 1987. CIE Guides ins. import. man. 1. Lepidoptera, p. 22.- Bridges, 1988. Cat. Fam.Group \& Gen.-Group Nam. 2, p. 1.- Scoble, 1989, in Emmet \& Head. Moths and Butt. Great Brit. and Ireland 7, p. 16, 17, 18, 35.- Minet, 1991. Ent. scand. 22: 76, 78.- Scoble, 1992. Lep., p. 190, 302.- Minet, 1994. Ent. scand. 25: 65.de Jong; Vane-Wright \& Ackery, 1996. Ent. scand. 27: 67.

Rhopalocères [sic]; Duméril, 1823. Dict. sc. nat. 26, p. 43; syn.: Globulicornes.- Boisduval, 1832. Icon. hist. Lép. d'Europe 1, p. 11; [divisão] oposta à Hétérocères.- Boisduval, 1836. Hist. nat. Ins. Sp. gén. Lép. 1, p. 162; legião oposta de Heterocères.- Blanchard, 1850, in Castelnau. Hist. nat. Anim. art. 3(2), p. 419.- Bar, 1878. Ann. Soc. ent. France (5)8: 11, 12, 13; oposto a Blastérocères - não os define, e Hétérocéres.Acloque, 1897. Faune France, p. 228.- Rambur, 1942. Faune ent. de L'Andalousie. $2^{\text {a }}$ ed., p. 238; divisão; syn.: Diurnes.Kiriakoff, 1946. Lambillionea 46: 62, 113.

Hexapoda Latreille, 1825. Fam. nat. règne anim., p. 467; sem status, incluindo [Papilionidae, Pieridae].- Westwood, 1840. Introd. mod. class. ins. 2, p. 338.- Doherty, 1886. Jour. Asiatic Soc. Bengal 55(2): 108, 110, 135; inclue Pieridae, Papilionidae, Hesperiadae.

Perlata Latreille, 1825. Fam. nat. règne anim., p. 468; sem status, incluindo [Nymphalidae].

Argus Latreille, 1825. Fam. nat. règne anim., p. 468; sem status, incluindo [Lycaenidae, Riodinidae].

Hesperi-Sphinges Latreille, 1825. Fam. nat. règne anim., p. 470; tribo de Crepuscularia, incluindo [Castniidae, Agaristidae].

Clavicornes Latreille, 1825. Fam. nat. règne anim., p. 471; syn.: Diurnes; sem status.

Pseudo-Bombyces Latreille, 1825. Fam. nat. règne anim., p. 473; syn.: Noctuo-Bombycites; tribo, incluindo [Cossidae,
Notodontidae, Arctiidae].- Latreille, 1829, in Cuvier. Règne Anim. 5, p. 403.- Guenée, 1875. Stat. sc. d'Eure-et-Loir. Lép., p. 151; legião, incluindo Tortriciformes, Erectae.

Phalaenites Latreille, 1825. Fam. nat. règne anim., p. 477; tribo, incluindo [Noctuidae].- Guenée, 1887, in Boisduval \& Guenée. Hist. nat. Ins. Sp. gén. Lép. 9, p. XLIX, 25; syn.: Geometra L.; grupo, incluindo Urapterydae, Ennomidae, Oenochromidae, Amphidasydae, Boarmidae, Boletobidae, Geometridae, Mecoceridae, Palyadae, Ephyridae, Acidalidae, Micronidae, Caberidae, Macaridae, Fidonidae, Hazidae, Zerenidae, Ligidae, Hybernidae, Larentidae, Eubolidae, Sionidae, Erateinidae, Emplocidae, Hypochrosidae.

Alis farinaceis Kirby \& Spence, 1826. Introd. Ent. 4, p. 432; syn.: Lepidoptera.

Tetrapodes $[=$ Tetrapoda] Latreille, 1829, in Cuvier. Règne Anim. 5, p. 375; série, incluindo Satyres, Pavonies, Morphos, Nymphales, Argynnes, Cethosies aux Heliconiens.- Burmeister, 1829. Insect. syst. nat., p. 27; divisão de Papilionacea, incluindo Erycina, Hipparchia, Apatura, Charaxes, Liminitis, Vanessa, Pavonia, Brassolis, Satyrus, Eurybia, Danaus, Idea, Acraea, Heliconius.- Zetterstedt, 1839. Ins. Lapponica, p. 885; seção, incluindo [Nymphalidae].- Bar, 1878. Ann. Soc. ent. France (5)8: 13.- Haase, 1891. Dtsch. ent. Ztschr. "Iris" 4: 5.

Hexapodes [sic]; Latreille, 1829, in Cuvier. Règne Anim. 5, p. 374; série, incluindo Papilio.- Burmeister, 1829. Insect. syst. nat., p. 27; divisão de Papilionacea, incluindo Barbicornia, Myrina, Lycaena, Pieris, Thais, Parnassius, Papilio.- Zetterstedt, 1839. Ins. Lapponica, p. 885; seção, incluindo [Papilionidae, Pieridae, Lycaenidae, Hesperiidae].- Bar, 1878. Ann. Soc. ent. France (5)8: 13.

Hepialites Latreille, 1829, in Cuvier. Règne Anim. 5, p. 397; seção, incluindo Hepialus (= Hepiolus) e Cossus.

Aposura Latreille, 1829, in Cuvier. Règne Anim. 5, p 407; seção, incluindo Dacronoura $(=$ Cerura, = Harpyia $)$, Platypterix (= Drepana).

Phalaenae Tortrices Latreille, 1829, in Cuvier. Règne Anim. 5, p. 411; seção, incluindo Pyralis.

Papilionacea Burmeister, 1829. Insect. syst. nat., p. 27; família, incluindo Tetrapodes e Hexapodes [= Papilionoidea + Hesperioidea].

Pomeridiana Stephens, 1829. Ill. Brit. Ent., Haustellata 2, p. 2; seção de Nocturna, incluindo Hepialidae, Bombycidae, Notodontidae, Arctiidae.- Westwood, 1840. Introd. mod. class. ins. 2 , p. 325,361 .

Semidiurna Stephens, 1829. Ill. Brit. Ent., Haustellata 3, p. 140; seção de Nocturna, incluindo Geometridae, Platyptericidae, Pyralidae.- Westwood, 1840. Introd. mod. class. ins. 2, p. $325,361$.

Papillonides [sic]; Boisduval \& Leconte, 1929. Hist. gén. icon. Lép. Chen. l'Amer. sept., p. 2; syn.: Papilionides; tribo, incluindo [Papilionidae]. 
Succinti Boisduval, 1832. Icon. hist. Lép. d'Europe 1, p. 11; subdivisão da divisão Rhopalocères, incluindo Papilionides, Lycénides.- Boisduval, 1836. Hist. nat. Ins. Sp. gén. Lép. 1, p. 162, 163.- Zetterstedt, 1839. Ins. Lapponica, p. 885.Boisduval, 1840. Gen. Ind. meth. Europ. Lep., p. 1, 15.Westwood, 1840. Introd. mod. class. ins. 2, p. 345.- Poey, 1846. Mem. r. Soc. econ. La Habana (2)2: 233.- Newman, 1870. Entomol. 5: 39, 42; sem status, incluindo Onisciformes, Cylindracei.

Penduli Boisduval, 1832. Icon. hist. Lép. d'Europe 1, p. 84; subdivisão da divisão Rhopalocères, incluindo Danaides, Nymphalides, Satyrides.

Heterocères [= Heterocera $]$ Boisduval, 1834. Icon. hist. Lép. d'Europe 2, p. 5; divisão, oposta à Rhopalocères.- Boisduval, 1836. Hist. nat. Ins. Sp. gén. Lép. 1, p. 162; legião oposta de Rhopalocères.- Blanchard, 1850, in Castelnau. Hist. nat. Anim. art. 3(2), p. 471, 481.- Guenée, 1852, in Boisduval \& Guenée. Hist. nat. Ins. Sp. gén. Lép. 5, p. 1.- Guenée, 1854, in Boisduval \& Guenée. Hist. nat. Ins. Sp. gén. Lép. 8, p. 1.- Acloque, 1897. Faune France, p. 228.- Lameere, 1931. Rec. Inst. zool. Torley-Rousseau 3(2): 235.- Lameere, 1938. Rec. Inst. zool. Torley-Rousseau 5: 169, 173, 174, 175, 176, 178.- Kiriakoff, 1946. Lambillionea 46: 62.

Vespertina Stephens, 1834. Ill. Brit. Ent., Haustellata 4, p. 63; [seção] de Nocturna, incluindo Tortricidae, Yponomeutidae, Tineidae, Alucitidae.- Westwood, 1840. Introd. mod. class. ins. 2, p. 325, 361, 362 .

Papilionina Newman, 1834. Ent. Mag. 2: 379, 381; estirpe, inluindo Papilionites e Hesperites [= Rhopalocera].- Lederer, 1853. Verh. zool.-bot. Ges. Wien 25: 16, 17.- Meyrick, 1895. Handb. brit. Lep., p. 21, 22, 325.- J. H. Comstock \& A. B. Comstock, 1904. Man. Study Ins. $4^{\text {a }}$ ed., p. 207.- Meyrick, 1928. Rev. Handb. brit. Lep., p. 29, 336.- Börner, 1920, in Brohmer. Fauna Deutschland. $2^{\mathrm{a}}$ ed., p. 354.- Le Marchand, 1945. Rev. franç. Lep. 10: 110.- Turner, 1947. Proc. Linn. Soc. N. S. Wales 71: 304, 305.

Suspensi Boisduval, 1836. Hist. nat. Ins. Sp. gén. Lép. 1, p. 162, 164; seção, incluindo Danaides, Héliconides, Nymphalides, Brassolides, Morphides, Satyrides, Biblides, Libythides.- Westwood, 1840. Introd. mod. class. ins. 2, p. 345.- Newman, 1870. Entomol. 5: 38, 41, 42; sem status, incluindo Spinigeri, Limaciformes.- Haase, 1891. Dtsch. ent. Ztschr. "Iris" 4: 3, 6.

Heterocera; Boisduval, 1840. Gen. Ind. meth. Europ. Lep., p. 39; legião, incluindo as mariposas.- Westwood, 1840. Introd. mod. class. ins. 2, p. 329, 361.- Herrich-Schäffer, 1849. Syst. Bearb. Schmett. Europa 4, p. 1.- HerrichSchäffer, [1858]. Samml. aussereurop. Schmett. 1, p. 56.Stainton, 1857. Man. Brit. Butt. and Moths 1, p. 72.Newman, 1870. Entomol. 5: 34.- Wallengren, 1872. Öfver. kungl. Vetensk.-Ak. Förh. 28: 961.- Burmeister, 1878. Descr. phys. Rép. Arg. 5, p. 44.- Reuter, 1896. Acta Soc. Sc.
Fenn. 22(1): 206, 213.- Snellen, 1882. Tijdschr. Ent. 25: 215.- Plötz, 1886. Mitt. naturw. Ver. Neu-Vorpomm. u. Rügen 17: 485, 492.- Chapman, 1893. Trans. ent. Soc. London, p. 98, 99, 118.- Chapman, 1895. Ent. Rec. \& Jour. Var. 6: 101, 104.- Packard, 1895. Mem. Nat. Ac. Sc. 7: 56.Karsch, 1898. Ent. Nachr. 24: 297, 298, 300, 301.- Holland, 1898. Butt. Book, p. 60.- Spuler, 1908. Schmett. Europas 1, p. 77.- Heymons, 1915, in zur Srassen. Brehms Tierleben. $4^{\mathrm{a}}$ ed., p. XX, 269.- Lindsey, 1921. Univ. Iowa Stud. Nat. Hist. 9(4): 6.- Börner, 1939. Verh. VII intern. Kongr. Ent. 2, p. 1403, 1407, 1422.- W. Ph. Comstock, 1944. Sc. Surv. P. Rico and Virgin Isl. 12(4), p. 428.- Kiriakoff, 1946. Lambillionea 46: 112.- Turner, 1947. Proc. Linn. Soc. N. S. Wales 71: 303.- Hayward, 1948. Gesp. nov. Anim. Arg. 1, p. 8.- Kiriakoff, 1948. Biol. Jaarboek, Antwerpen, 15, p. 140.- Börner, 1949, in Brohmer. Fauna Deutschland. $6^{a}$ ed., p. 397.- Hennig, 1953. Beitr. Ent. 3, Sonderh., p. 47.Richards \& Davies, 1957, in Imms. Gen. Textb. Ent., p. 533.Friese, 1969. Bericht 10. Wandervers. Deutscher Ent., Tagungsb. 80(2): 205, 207.- Razowski, 1974. Acta zool. cracov. 19: 8.- Common, 1975. Ann. Rev. Ent. 20: 183.Gómez B., 1978. Shilap, Revta lepid. 6: 249.- Scoble, 1989, in Emmet \& Head. Moths and Butt. Great Brit. and Ireland 7, p. 17, 18.- Scoble, 1992. Lep., p. 190.

Succinctae [sic]; Boisduval, 1840. Gen. Ind. meth. Europ. Lep., p. 1; divisão, sem status, de Rhopalocera, oposta à Pendulae e Involutae [Hesperiidae].- Plötz, 1886. Mitt. naturw. Ver. NeuVorpomm. u. Rügen 17: 485, 491.- de Jong; Vane-Wright \& Ackery, 1996. Ent. scand. 27: 66.

Pendulae [sic]; Boisduval, 1840. Gen. Ind. meth. Europ. Lep., p. 1, 15; divisão, sem status, de Rhopalocera, incluindo [Nymphalidae].- Plötz, 1886. Mitt. naturw. Ver. NeuVorpomm. u. Rügen 17: 488; na sinonímia de Nymphalidae.de Jong; Vane-Wright \& Ackery, 1996. Ent. scand. 27: 66.

Microlepidopteren [= Microlepidoptera] ???.- Herrich-Schäffer, 1840, in Fürnrohr. Naturhistorische Topographie von Regensburg 3, p. 50 (Microlepidoptern - [sic]), 149.- Herrich-Schäffer, 1849. Syst. Bearb. Schmett. Eur. 4, p. 1.- Herrich-Schäffer, 1849. Corr.-Blatt zool.-min. Ver. Regensburg 3: 26, 55, 154, 156, 158, 167, 168.- Herrich-Schäffer, 1852. Corr.-Blatt zool.min. Ver. Regensburg 6: 18, 19, 21, 32, 189.

Tetrapoda [sic]; Westwood, 1840. Introd. mod. class. ins. 2, p. 338.- Doherty, 1886. Jour. Asiatic Soc. Bengal. 55(2): 108, 113.

Nudi Westwood, 1840. Introd. mod. class. ins. 2, Synopsis gen. brit. ins., p. 87; subseção [= Papilionoidea].- Haase, 1891. Dtsch. ent. Ztschr. "Iris" 4: 6.- D'Almeida, 1937. Mem. Inst. Osw. Cruz 32(2): 246.- D'Almeida, 1966. Cat. Papilionidae amer., p. 4.

Nocturnae [sic]; Gravenhorst, 1843. Vergleichende Zool., p. 167. Microlepidoptera ???.- Herrich-Schäffer, 1845. Syst. Bearb. Schmett. Europa 2, p. 2.- Herrich-Schäffer, 1849. Syst. 
Bearb. Schmett. Europa 4, p. 1; sem status, incluindo Pyralis, Tortrix, Tinea, Alucita.- Herrrich-Schäffer, 1856-[1858]. Samml. aussereurop. Schmett. 1, p. 5 (1856), p. 74 ([1858]).- Koch, 1856. Schmett. südwestl. Dtschl., p. 295.- Burmeister, 1878. Descr. phys. Rép. Arg. 5, p. 45.- Dyar, 1894. Ann. N. Y. Acad. Sc. 8: 205.- Dyar, 1895. Ent. News 6: 39; superfamília.- Dyar, 1895. Jour. N. Y. Ent. Soc. 3: 25.- Dyar, 1895. Trans. N. Y. Ac. Sc. 14: 53.- Chapman, 1896. Trans. ent. Soc. London, p. 579.- Tutt, 1897. Ent. Rec. \& Jour. Var. 9: 194.- Karsch, 1898. Ent. Nachr. 24: 297, 301.- Tutt, 1899. Brit. Lep. 1, p. 107.Fracker, 1915. Illinois Biol. Monogr. 2: 29, 45.- Hennig, 1953. Beitr. Ent. 3, Sonderh., p. 47.- Richards \& Davies, 1957, in Imms. Gen. Textb. Ent., p. 533.- Friese, 1969. Bericht 10. Wandervers. Deutscher Ent., Tagungsb. 80(2): 205.- Brock, 1971. Jour. nat. Hist. 5: 83-99.- Razowski, 1974. Acta zool. cracov. 19: 8, 9.- Kuitjen, 1974. Neth. Jour. Zool. 24: 319, 320.- Common, 1975. Ann. Rev. Ent. 20: 183.- Gómez B., 1978. Shilap, Revta lepid. 6: 250.- Holloway; Bradley \& Carter, 1987. CIE Guides ins. import. man. 1. Lepidoptera, p. 77.- Scoble, 1992. Lep., p. 190.- K. Brown, 1996, in Bicudo \& Menezes. Biodiv. Brasil, p. 224.

Achalinoptères [= Achalinoptera] Blanchard, 1845. Hist. Ins. 2, p. 322, 323; seção oposta à Chalinoptères, incluindo [Papilionoidea e Hesperioidea].- Lucas, 1864. Hist. nat. Lép. d'Europe, p. 21; syn.: Achalinoptera.

Chalinoptères [= Chalinoptera] Blanchard, 1845. Hist. Ins. 2, p. 322,323 ; seção, incluindo as mariposas, oposta à Achalinoptères.- Lucas, 1864. Hist. nat. Lép. d'Europe, p. 120; syn.: Chalinoptera.

Papilioniens Blanchard, 1845. Hist. Ins. 2, p. 324, 325; tribo, incluindo Papilionides, Piérides.

Nymphaliens Blanchard, 1845. Hist. Ins. 2, p. 329, 330; tribo, incluindo Nymphaliens.

Eryciniens Blanchard, 1845. Hist. Ins. 2, p. 341, 342; tribo, incluindo Lycénites, Erycinites.

Cydimoniens Blanchard, 1845. Hist. Ins. 2, p. 348, 349; tribo sem família, incluindo os gêneros Cydimon, Nyctalémon.

Castniens Blanchard, 1845. Hist. Ins. 2, p. 349, 350; tribo, incluindo Agaristites, Castnites.

Sésiens Blanchard, 1845. Hist. Ins. 2, p. 351, 352; tribo, incluindo Chimérides, Sésiides.

Zygaeniens Blanchard, 1845. Hist. Ins. 2, p. 353, 354; tribo, incluindo Procrites, Zygénites.

Sphingiens Blanchard, 1845. Hist. Ins. 2, p. 356, 357; tribo sem família, incluindo [Sphingidae].

Bombyciens Blanchard, 1845. Hist. Ins. 2, p. 360, 361; tribo, incluindo Bombycites, Psychides, Platyptérides, Hépialides, Notodontides.

Noctuéliens Blanchard, 1845. Hist. Ins. 2, p. 379, 380; tribo, incluindo Noctuides.

Uraniens Blanchard, 1845. Hist. Ins. 2, p. 390; tribo, incluindo [Uraniidae].
Phaléniens Blanchard, 1845. Hist. Ins. 2, p. 391, 392; tribo, incluindo [Geometridae].

Pyraliens Blanchard, 1845. Hist. Ins. 2, p. 399; tribo, incluindo Botydes, Pyralides, Crambides, Tinéides.

Microlopidopteren [sic]; Herrich-Schäffer, 1849. Corr.-Blatt zool.-min. Ver. Regensburg 3: 166.

Crepusculaires [sic]; Blanchard, 1850, in Castelnau. Hist. nat. Anim. art. 3(2), p. 471.- Duméril, 1860. Ent. analyt. 2, p. 1090.

Macrolepidoptera ???.- Herrich-Schäffer, 1852. Corr.-Blatt zool. min. Ver. Regensburg 6: 18 [Gross-Schmetterlinge = "Macrolepidoptera"].- Koch, 1856. Schmett. südwestl. Dtschl., p. 1.- Dyar, 1894. Ann. N. Y. Ac. Sc. 8: 205, 224.Packard, 1895. Mem. Nat. Ac. Sc. 7: 62; syn.: Platylepidoptera [inclue os grupos com asas largas].- Packard, 1895. Zool. Anz. 18: 235; syn.: Platylepidoptera.- Chapman, 1896. Trans. ent. Soc. London, p. 575, 576.- Tutt, 1897. Ent. Rec. \& Jour. Var. 9: 194.- Karsch, 1898. Ent. Nachr. 24: 297, 301.Handlirsch, 1906. Die fossilen Ins. 1, p. 44.- Fracker, 1915. Illinois Biol. Monogr. 2: 58.- Hennig, 1953. Beitr. Ent. 3, Sonderh., p. 47.- Richards \& Davies, 1957, in Imms. Gen. Textb. Ent., p. 533.- Friese, 1969. Bericht 10. Wandervers. Deutscher Ent., Tagungsb. 80(2): 205.- Brock, 1971. Jour. nat. Hist. 5: 82-99.- Razowski, 1974. Acta zool. cracov. 19: 8.- Kuitjen, 1974. Neth. Jour. Zool. 24: 319, 320.- Common, 1975. Ann. Rev. Ent. 20: 183.- Gómez B., 1978. Shilap, Revta lepid. 6: 250.- Holloway; Bradley \& Carter, 1987. CIE Guides ins. import. man. 1. Lepidoptera, p. 133.- Scoble, 1989. Moths and Butt. Great Brit. and Ireland 7, p. 34.Minet, 1991. Ent. scand. 22: 72, 74, 81, 83.- Scoble, 1992. Lep., p. 190, 191.- Minet, 1994. Ent. scand. 25: 65.- K. Brown, 1996, in Bicudo \& Menezes. Biodiv. Brasil, p. 225.Poole, 1996, in Poole \& Gentili. Nom. Ins. Nearct. 3, p. 619.- Kristensen \& Skalski, 1999, in Kristensen (Ed.), Lep. 1, p. 10, 14, in Fischer (Ed.). Handb. Zool. 4.- Carter \& Kristensen, 1999, in Kristensen (Ed.), Lep. 1, p. 28, in Fischer (Ed.). Handb. Zool. 4.- Powell et al., 1999, in Kristensen (Ed.), Lep. 1, p. 412, in Fischer (Ed.). Handb. Zool. 4.

Micrognatha Spinola, 1852. Mem. Mat. Fis. Soc. ital. Sc. Modena 25(1): 66; syn.: Lepidoptera, Glossata; ordem.

Acalinopteros [sic]; Blanchard, 1852, in Gay. Hist. fis. pol. Chile 7 , p. 6.

Noctuélites Guenée, 1852, in Boisduval \& Guenée. Hist. nat. Ins. Sp. gén. Lép. 5, p. 1; syn.: Noctuae L.; divisão, incluindo as falanges Trifidae, Quadrifidae.

Trifidae Guenée, 1852, in Boisduval \& Guenée. Hist. nat. Ins. Sp. gén. Lép. 5, p. 5; falange, incluindo Noctuélites, Deltoïdes, Phalenites.- Stainton, 1857. Man. Brit. Butt. and Moths 1, p. 171, 172; grupo, incluindo Bombyciformes, Genuinae, Minores.- Guenée, 1875. Stat. sc. d'Eure-et-Loir. Lép., p. 164; divisão, incluindo Bombyciformes, Genuinae, Minores.

Bombyciformes Guenée, 1852, in Boisduval \& Guenée. Hist. nat.

Revista Brasileira de Zoologia 23 (1): 1-41, março 2006 
Ins. Sp. gén. Lép. 5, p. 5, 7; tribo, incluindo NoctuoBombycidae, Bryophilidae, Bombycoidae.- Stainton, 1857. Man. Brit. Butt. and Moths 1, p. 171, 172.- Newman, 1870. Entomol. 5: 40, 42.- Guenée, 1875. Stat. sc. d'Eure-et-Loir. Lép., p. 164; tribo, incluindo Thyatyridae, Cymatophorides, Bryophilidae, Acronyctidae.- Lameere, 1936. Bull. \& Ann. Soc. ent. Belg. 76: 412, 413; grupo, incluindo Bombycoïdes, Sphingoïdes.- Lameere, 1938. Rec. Inst. zool. TorleyRousseau 5: 194.- Heppner, 1984. Atlas neotrop. Lep. 2. Checkl. 1, p. XXII; série de Bombycoidea, infra-superfamíla.Heppner, 1995. Atlas neotrop. Lep. 3. Checkl. 2, p. XIV; série de Bombycoidea, infra-superfamília.- Heppner, 1996. Atlas neotrop. Lep. 5B. Checklist 4B, p. XVI; série de Bombycoidea, infra-superfamília.- Heppner, 1998. Lep. News 1998 (3): 59.- Heppner, 1998. Holarct. Lep. 5, Suppl. 1: 21. Genuinae Guenée, 1852, in Boisduval \& Guenée. Hist. nat. Ins. Sp. gén. Lép. 5, p. 6, 63; tribo, incluindo Leucanidae, Glottalidae, Apamidae, Caradrinidae, Noctuidae, Orthosidae, Hadenidae, Cosmidae, Xylinidae, Heliothidae.-Stainton, 1857. Man. Brit. Butt. and Moths 1, p. 171, 172; seção, incluindo "seven-eights of the European Noctuae".- Guenée, 1875. Stat. sc. d'Eure-et-Loir. Lép., p. 171; tribo, incluindo Leucanidae, Gortynidae, Apamidae, Caradrinidae, Episemidae, Noctuides, Orthosidae, Cosmidae, Hadenidae, Xylinidae, Cucullidae, Heliothidae.

Minores Guenée, 1852, in Boisduval \& Guenée. Hist. nat. Ins. Spec. gén. Lép. 5, p. 6; 6, p. 199; tribo, incluindo Haemerosidae, Acontididae, Erastridae, Anthophilidae, Phalaenoidae.- Stainton, 1857. Man. Brit. Butt. and Moths 1, p. 171, 172; seção, incluindo "imago of small size".Guenée, 1875. Stat. sc. d'Eure-et-Loir. Lép., p. 223; tribo, incluindo Acontidae, Erastridae, Brephidae.

Quadrifidae Guenée, 1852, in Boisduval \& Guenée. Hist. nat. Ins. Sp. gén. Lép. 6, p. 267; falange, incluindo Sericeae, Variegatae, Intrusae, Extensae, Limbatae, Patulae, Serpentinae, Pseudo-Deltoïdae.- Stainton, 1857. Man. Brit. Butt. and Moths 1, p. 171; grupo, incluindo Variegatae, Intrusae, Limbatae, Serpentinae.- Guenée, 1875. Stat. sc. d'Eure-etLoir. Lép., p. 228; divisão, incluindo Variegatae, Intrusae, Limbatae, Serpentinae.

Sericeae Guenée, 1852, in Boisduval \& Guenée. Hist. Nat. Ins. Sp. gén. Lép. 6, p. 273; tribo, incluindo Palindidae, Dyopsidae.

Variegatae Guenée, 1852, in Boisduval \& Guenée. Hist. nat. Ins. Sp. gén. Lép. 6, p. 286; tribo, incluindo Eriopidae, Eurhipidae, Placodidae, Plusidae, Calpidae, Hemiceridae, Hyblaeidae, Gonopteridae.- Stainton, 1857. Man. Brit. Butt. and Moths 1, p. 171; seção de Quadrifidae, sem incluir taxon.- Guenée, 1875. Stat. sc. d'Eure-et-Loir. Lép., p. 228; tribo, incluindo Plusidae, Gonopterygidae.

Intrusae Guenée, 1852, in Boisduval \& Guenée. Hist. nat. Ins. Sp. gén. Lép. 6, p. 407; tribo, incluindo Amphipyridae, Toxocampidae, Stilbidae.- Stainton, 1857. Man. Brit. Butt. and Moths 1, p. 171; seção de Quadrifidae, sem incluir taxon.- Guenée, 1875. Stat. sc. d'Eure-et-Loir. Lép., p. 229; tribo, incluindo Amphypiridae, Maniidae, Toxocampidae.

Extensae Guenée, 1852, in Boisduval \& Guenée. Hist. nat. Ins. Sp. gén. Lép. 6, p. 435; tribo, incluindo Polydesmidae, Homopteridae, Hypogrammidae.

Limbatae Guenée, 1852, in Boisduval \& Guenée. Hist. nat. Ins. Sp. gén. Lép. 7, p. 39; tribo, incluindo Catephidae, Bolinidae, Hypocalidae, Catocalidae, Ophideridae.- Stainton, 1857. Man. Brit. Butt. and Moths 1, p. 171; seção de Quadrifidae, sem incluir taxon.- Guenée, 1875. Stat. sc. d'Eure-et-Loir. Lép., p. 234; tribo, incluindo Catephidae, Catocalidae.

Patulae Guenée, 1852, in Boisduval \& Guenée. Hist. nat. Ins. Sp. gén. Lép. 7, p. 126; tribo, incluindo Erebidae, Ommatophoridae, Hypopyridae, Bendidae.

Serpentinae Guenée, 1852, in Boisduval \& Guenée. Hist. nat. Ins. Sp. gén. Lép. 7, p. 219; tribo, incluindo Ophiusidae, Euclididae, Poaphilidae, Remigidae.- Stainton, 1857. Man. Brit. Butt. and Moths 1, p. 171; seção de Quadrifidae, sem incluir taxon.- Guenée, 1875. Stat. sc. d'Eure-et-Loir. Lép., p. 237; tribo, incluindo Ophiusidae, Euclididae, Poaphilidae.

Pseudo-Deltoidae Guenée, 1852, in Boisduval \& Guenée. Hist. nat. Ins. Sp. gén. Lép. 7, p. 327; tribo, incluindo Focillidae, Amphigonidae, Thermesidae.

Succintae B Lederer, 1853. Verh. zool.-bot. Ges. Wien 25: 16; sem status, incluindo [Papilionidae, Pieridae, Lycaenidae, Riodinidae].

Pendulae B Lederer, 1853. Verh. zool.-bot. Ges. Wien 25: 16; sem status, incluindo [Libytheidae, Nymphalidae, Danaidae, Satyridae].

Squamosae Guenée, 1854, in Boisduval \& Guenée. Hist. nat. Ins. Sp. gén. Lép. 8, p. 111; tribo, incluindo Odontidae.Stainton, 1859. Man. Brit. Butt. and Moths 2, p. 124; tribo, incluindo Odontidae.- Guenée, 1875. Stat. sc. d'Eureet-Loir. Lép., p. 246; tribo, incluindo Odontidae.

Pulverulentae Guenée, 1854, in Boisduval \& Guenée. Hist. nat. Ins. Sp. gén. Lép. 8, p. 115; tribo, incluindo Pyralidae, Cledeobidae.- Stainton, 1859. Man. Brit. Butt. and Moths 2, p. 124; tribo, incluindo Pyralidae, Cledeobidae.- Guenée, 1875. Stat. sc. d'Eure-et-Loir. Lép., p. 247; tribo, incluindo Pyralidae, Cledeobidae.

Luridae Guenée, 1854, in Boisduval \& Guenée. Hist. nat. Ins. Sp. gén. Lép. 8, p. 145; tribo, incluindo Hercynidae, Ennychidae, Asopidae, Steniadae, Hydrocampidae, Spilomelidae, Margarodidae, Botydae.-Stainton, 1859. Man. Brit. Butt. and Moths 2, p. 124; tribo, incluindo Ennychidae, Asopidae, Steniadae, Hydrocampidae, Botydae, Nolidae, Choreutidae.Guenée, 1875. Stat. sc. d'Eure-et-Loir. Lép., p. 251; tribo, incluindo Hercynidae, Eunychidae, Asopidae, Steniadae, Hydrocampidae, Botydae.

Plicatae Guenée, 1854, in Boisduval \& Guenée. Hist. nat. Ins. Sp. gén. Lép. 8, p. 412; tribo, incluindo Scoparidae.-

Revista Brasileira de Zoologia 23 (1): 1-41, março 2006 
Stainton, 1859. Man. Brit. Butt. and Moths 2, p. 125; tribo, incluindo o gênero Eudorea.

Saturniina Herrich-Schäffer, 1856, [1858]. Samml. aussereurop. Schmett. 1, p. 4 (1856), p. 60 [1858]; família, incluindo [Saturniidae].

Micropterygina Herrich-Schäffer, 1856. Samml. aussereurop. Schmett. 1, p. 5; família, incluindo [Micropterigidae].- Meyrick, 1895. Handb. brit. Lep., p. 21, 22, 797; grupo, incluindo Micropterygidae, Hepialidae.- J. H. Comstock, 1918. Wings Ins., p. 313, 315; subordem de Trichoptera.- Meyrick, 1928. Rev. Handb. brit. Lep., p. 29, 865.- Börner, 1939. Verh. VII intern. Kongr. Ent., Berlin, 2, p. 1407, 1423.- Le Marchand, 1945. Rev. franç. Lép. 45: 110.- Kiriakoff, 1946. Lambillionea 46: 22.- Turner, 1947. Proc. Linn. Soc. N. S. Wales 71: 304, 305.Common, 1975. Ann. Rev. Ent. 20: 183.

Sphingina Herrich-Schäffer, 1856, [1858]. Samml. aussereurop. Schmett. 1, p. 3 (1856), p. 59 [1858]; família, incluindo [Sphingidae].- Stainton, 1857. Man. Brit. Butt. and Moths 1, p. 72, 75; grupo, incluindo Zygaenidae, Sphingidae, Sesiidae, Aegeriidae.- Dyar, 1895. Trans. N. Y. Ac. Sc. 14: 53, 59, 62.Dyar, 1895. Ent. News 6: 39; superfamília.- Dyar, 1895. Jour. N. Y. Ent. Soc. 3: 26.- Tutt, 1899. Brit. Lep. 1, p. 107, 109.Turner, 1947. Proc. Linn. Soc. N. S. Wales 71: 303.

Psychina Herrich-Schäffer, 1856. Samml. aussereurop. Schmett. 1, p. 3; família, incluindo [Psychidae].- Meyrick, 1895. Handb. brit. Lep., p. 21, 22, 442; grupo, incluindo Zeuzeridae, Zygaenidae, Psychidae, Heterogeneidae.- Meyrick, 1928. Rev. Handb. brit. Lep., p. 29, 469.- Le Marchand, 1945. Rev. franç. Lép. 10: 110.- Turner, 1947. Proc. Linn. Soc. N. S. Wales 71: 304, 305.

Lasiocampina Herrich-Schäffer, 1856. Samml. aussereurop. Schmett. 1, p. 3; família, incluindo [Lasiocampidae].Meyrick, 1895. Handb. brit. Lep., p. 21, 22, 314; grupo, incluindo Drepanidae, Endromididae, Lasiocampidae.Meyrick, 1928. Rev. Handb. brit. Lep., p. 29, 461.- Le Marchand, 1945. Rev. franç. Lép. 10: 110.- Turner, 1947. Proc. Linn. Soc. N. S. Wales 71: 304, 305.

Cossina Herrich-Schäffer, 1856, [1858]. Samml. aussereurop. Schmett. 1, p. 3 (1856), p. 58 ([1858]); família, incluindo [Cossidae].- Dyar, 1895. Trans. N. Y. Ac. Sc. 14: 53, 61; superfamília, incluindo Adelidae, Psychidae, Cossidae, Pyralidae (= Pyralidina), Tortricidae (= Tortricina), Sesiidae, Tineidae (= Tineina), Orneodidae, Lacosomidae.- Dyar, 1895. Jour. N. Y. Ent. Soc. 3: 25.- Heppner, 1984. Atlas neotrop. Lep. 2. Checkl. 1, p. XXI (seção).- Heppner, 1995. Atlas neotrop. Lep. 3. Checkl. 2, p. XIV.- Heppner, 1996. Atlas neotrop. Lep. 5B. Checklist 4B, p. XVI; seção de Ditrysia e subseção de Cossina.- Heppner, 1998. Lep. News 1998 (3): 58; seção e subseção.- Heppner, 1998. Holarct. Lep. 5, Suppl. 1: 7, 20; seção e subseção.- Heppner, 2003. Lep. Florida 1, p. 73, 92, 94, 231; seção e subseção.

Notodontina Herrich-Schäffer, 1856, [1858]. Samml. aussereurop. Schmett. 1, p. 4 (1856), p. 66 ([1858)]; famí- lia, incluindo [Notodontidae].- Meyrick, 1895. Handb. brit. Lep., p. 21, 22, 176; grupo, incluindo Polyplocidae, Saturniadae, Notodontidae, Selidosemidae, Sphingidae, Geometridae, Sterrhidae, Monocteniadae, Hydriomenidae.Meyrick, 1928. Rev. Handb. brit. Lep., p. 29, 194.- Le Marchand, 1945. Rev. franç. Lép. 10: 110.- Turner, 1947. Proc. Linn. Soc. N. S. Wales 71: 304, 305.

Noctuina Herrich-Schäffer, 1856, [1858]. Samml. aussereurop. Schmett. 1, p. 4 (1856), p. 67 ([1858]); família, incluindo [Noctuidae].- Stainton, 1857. Man. Brit. Butt. and Moths 1, p. 72, 170; grupo, incluindo Trifidae, Quadrifidae.- Dyar, 1895. Trans. N. Y. Ac. Sc. 14: 53, 54, 61.- Dyar, 1895. Ent. News 6: 39; superfamília.- Dyar, 1895. Jour. N. Y. Ent. Soc. 3: 26.- Chapman, 1896. Trans. ent. Soc. London, p. 587; syn.: Caradrinina.- Tutt, 1899. Brit. Lep. 1, p. 107, 109.Turner, 1947. Proc. Linn. Soc. N. S. Wales 71: 303.

Pterophorina Herrich-Schäffer, 1856. Samml. aussereurop. Schmett. 1, p. 5; família, incluindo [Pterophoridae].Stainton, 1857. Man. Brit. Butt. and Moths 1, p. 73; grupo.- Stainton, 1859. Man. Brit. Butt. and Moths 2, p. 439; grupo, incluindo Adactyla, Pterophorus [gêneros].- Turner, 1947. Proc. Linn. Soc. N. S. Wales 71: 303.

Alucitina Herrich-Schäffer, 1856. Samml. aussereurop. Schmett. 1, p. 5; família, incluindo [Alucitidae].- Stainton, 1857. Man. Brit. Butt. and Moths 1, p. 73; grupo.-Stainton, 1859. Man. Brit. Butt. and Moths 2, 445; grupo, incluindo Alucita.- Turner, 1947. Proc. Linn. Soc. N. S. Wales 71: 303.

Tineina Stainton, 1857. Man. Brit. Butt. and Moths 1, p. 73; grupo.- Stainton, 1859. Man. Brit. Butt. and Moths 2, p. 278; grupo, incluindo Exapatidae, Tineidae, Micropterygidae, Argyresthidae, Hyponomeutidae, Glyphipterygidae, Gelechidae, Oecophoridae, Gracilariidae, Elachistidae, Lithocolletidae, Lyonetidae, Nepticulidae, Plutellidae, Coleophoridae.Chapman, 1893. Trans. ent. Soc. London, p. 98, 99, 114.Meyrick, 1895. Handb. brit. Lep., p. 21, 22, 560.-Chapman, 1895. Ent. Rec. \& Jour. Var. 6: 105.- Meyrick, 1928. Rev. Handb. brit. Lep., p. 29, 598.- Le Marchand, 1945. Rev. franç. Lép. 10: 94, 100, 104, 110, 125, 130, 138.- Turner, 1947. Proc. Linn. Soc. N. S. Wales 71: 303, 305.- Heppner, 1984. Atlas neotrop. Lep. 2. Checkl. 1, p. XX.- Heppner, 1995. Atlas neotrop. Lep. 3. Checkl. 2, p. XIII.- Heppner, 1996. Atlas neotrop. Lep. 5B. Checkl. 4B, p. XV; seção de Ditrysia e subseção de Tineina.- Heppner, 1998. Lep. News 1998 (3): 58; seção e subseção.- Heppner, 1998. Holarct. Lep. 5, Suppl. 1: 7, 18; seção e subseção.- Heppner, 2003. Lep. Florida 1, p. 71, 91, 94, 231; seção e subseção.

Pyralidina Stainton, 1857. Man. Brit. Butt. and Moths 1, p. 73; grupo.- Stainton, 1859. Man. Brit. Butt. and Moths 2, p. 123; grupo, incluindo Pyralidous, Crambideous.- Meyrick, 1895. Handb. brit. Lep., p. 21, 22, 360.- Meyrick, 1928. Rev. Handb. brit. Lep., p. 29, 375.- Le Marchand, 1945. Rev. franç. Lép. 10: 110.- Turner, 1947. Proc. Linn. Soc. N. S. Wales 71: 303-305. 
Bombycina Stainton, 1857. Man. Brit. Butt. and Moths 1, p. 72, 107; grupo, incluindo Hepialidae, Zeuzeridae, Notodontidae, Liparidae, Lithosidae, Chelonidae, Bombycidae, Endromidae, Saturnidae, Platypterygidae, Psychidae, Cochliopodidae.- HerrichSchäffer, [1858]. Samml. aussereurop. Schmett. 1, p. 60; família, incluindo [Bombycidae].- Dyar, 1895. Ent. News 6: 39; superfamília.- Tutt, 1899. Brit. Lep. 1, p. 107, 109.Turner, 1947. Proc. Linn. Soc. N. S. Wales 71: 303.- Heppner, 1995. Atlas neotrop. Lep. 3. Checkl. 2, p. XIV.- Heppner, 1996. Atlas neotrop. Lep. 5B. Checkl. 4B, p. XVI; subseção de Cossina.- Heppner, 1998. Lep. News 1998 (3): 59.Heppner, 1998. Holarct. Lep. 5, Suppl. 1: 7, 20.- Heppner, 2003. Lep. Florida 1, p. 73, 93, 94, 336; subseção.

Geometrina Stainton, 1857. Man. Brit. Butt. and Moths 1, p. 73; grupo.- Stainton, 1859. Man. Brit. Butt. and Moths 2, p. 3; grupo, incluindo Ourapterydae, Ennomidae, Amphidasidae, Boarmidae, Boletobidae, Geometridae, Ephyridae, Acidalidae, Caberidae, Macaridae, Fidonidae, Zerenidae, Ligidae, Hybernidae, Larentidae, Eubolidae, Sionidae.- Turner, 1947. Proc. Linn. Soc. N. S. Wales 71: 303.

Tortricina Stainton, 1857. Man. Brit. Butt. and Moths 1, p. 73; grupo, incluindo Lozotaeniae, Sciaphilae, \&c..- HerrichSchäffer, [1858]. Samml. aussereurop. Schmett. 1, p. 75; família, incluindo [Tortricidae].- Stainton, 1859. Man. Brit. Butt. and Moths 2, p. 188; grupo, incluindo Tortricidae, Plicatae, Anchyloperidae, Peroneidae, Stigmonotidae, Carpocapsidae, Cnephasidae, Sericoridae, Lozoperidae.Chapman, 1893. Trans. ent. Soc. London, p. 98.- Meyrick, 1895. Handb. brit. Lep., p. 21, 22, 451.- Meyrick, 1928. Rev. Handb. brit. Lep., p. 29, 481.- Le Marchand, 1945. Rev. franç. Lép. 10: 110.- Turner, 1947. Proc. Linn. Soc. N. S. Wales 71: 304, 305.

Pyralideous Stainton, 1859. Man. Brit. Butt. and Moths 2, p. 124; grupo, incluindo Deltoides, Pyralites.

Crambideous Stainton, 1859. Man. Brit. Butt. and Moths 2, p. 124, 159; grupo, incluindo Eudoreidae, Galleridae, Phycidae, Crambidae.

Achalinoptera [sic]; Herrich-Schäffer, 1862. Corr.-Blatt zool.min. Ver. Regensburg 16: 174.

Chalinoptera [sic]; Herrich-Schäffer, 1863. Corr.-Blatt zool-min. Ver. Regensburg 17: 147.

Lepidopters [sic]; Dana, 1864. Amer. Jour. Sc. (2)37: 14, 15, 18,30-32.

Pedunculata Newman, 1870. Entomol. 5: 34, 38, 42; sem status, incluindo Detegentes, Celantes.

Detegentes Newman, 1870. Entomol. 5: 38, 41, 42; sem status, incluindo Suspensi, Succinti.

Celantes Newman, 1870. Entomol. 5: 40, 42; sem status, incluindo Bombyciformes, Capitati [= Hesperiidae], Synemonii, Cydimonii.

Sessiliventres Newman, 1870. Entomol. 5: 34, 38, 41; sem status, incluindo [Uraniidae].
Spinigeri Newman, 1870. Entomol. 5: 38, 42; sem status, incluindo Vanessidae, Argynnidae.

Limaciformes Newman, 1870. Entomol. 5: 39, 42; sem status, incluindo Apatura, Satyridae.

Onisciformes Newman, 1870. Entomol. 5: 39, 42; sem status, incluindo Erycinidae, Lycaenidae.- Guenée, 1875. Stat. sc. d'Eure-et-Loir. Lép., p. 30; legião, incluindo Micropi, Heteropi.

Cylindracei Newman, 1870. Entomol. 5: 39, 42; sem status, incluindo Papilionidae, Pieridae, Rhodoceridae.

Synemonii Newman, 1870. Entomol. 5: 40, 42; sem status, incluindo Hesperia (?) sophia White, theresa Doubleday, mopsa Doubleday, laeta Walker, plana Walker.

Cydemonii Newman, 1870. Entomol. 5: 40, 41, 42; sem status, incluindo [Uraniidae].

Bicalcarati Guenée, 1875. Stat. sc. d'Eure-et-Loir. Lép., p. 9; divisão, incluindo Fusiformes, Onisciformes.

Fusiformes Guenée, 1875. Stat. sc. d'Eure-et-Loir. Lép., p. 9; legião, incluindo Hexapi, Tetrapi.

Tentaculatae Guenée, 1875. Stat. sc. d'Eure-et-Loir. Lép., p. 10; tribo, incluindo Papilionidae.

Granulosae Guenée, 1875. Stat. sc. d'Eure-et-Loir. Lép., p. 11; tribo, incluindo Pieridae.

Spinosae Guenée, 1875. Stat. sc. d'Eure-et-Loir. Lép., p. 17; tribo, incluindo Argynnidae.

Carunculatae Guenée, 1875. Stat. sc. d'Eure-et-Loir. Lép., p. 23; tribo, incluindo Nymphalidae.

Furculae Guenée, 1875. Stat. sc. d'Eure-et-Loir. Lép., p. 25; tribo, incluindo Satyridae.

Micropi Guenée, 1875. Stat. sc. d'Eure-et-Loir. Lép., p. 30; falange, incluindo Sulcatae.

Sulcatae Guenée, 1875. Stat. sc. d'Eure-et-Loir. Lép., p. 30; tribo, incluindo Theclidae.

Heteropi Guenée, 1875. Stat. sc. d'Eure-et-Loir. Lép., p. 36; falange, incluindo Nemeobidae.

Areolati Guenée, 1875. Stat. sc. d'Eure-et-Loir. Lép., p. 41; divisão, incluindo Spinicornes, Prismaticornes, Lignivorae, Infrenatae, Apodae, Pellucidae, Crassicornes, Plicatulae, Plumicornes, Pecticornes, Phalaenidae, Pseudo-Bombyces, Noctuae, Deltoidae, Pyralidae.

Spinicornes Guenée, 1875. Stat. sc. d'Eure-et-Loir. Lép., p. 41; legião, incluindo Sesiidae.

Prismaticornes Guenée, 1875. Stat. sc. d'Eure-et-Loir. Lép., p. 42; legião, incluindo Sphingidae.

Lignivorae Guenée, 1875. Stat. sc. d'Eure-et-Loir. Lép., p. 50; legião, incluindo Zeuzeridae, Cossides.

Infrenatae Guenée, 1875. Stat. sc. d'Eure-et-Loir. Lép., p. 52; legião, incluindo Terricolae.

Terricolae Guenée, 1875. Stat. sc. d'Eure-et-Loir. Lép., p. 52; tribo, incluindo Hepialidae.

Apodae Guenée, 1875. Stat. sc. d'Eure-et-Loir. Lép., p. 54; legião, incluindo Cocliopodae.

Revista Brasileira de Zoologia 23 (1): 1-41, março 2006 
Pellucidae Guenée, 1875. Stat. sc. d'Eure-et-Loir. Lép., p. 55; legião, incluindo Saccophorae.

Saccophorae Guenée, 1875. Stat. sc. d'Eure-et-Loir. Lép., p. 55; tribo, incluindo Psychidae.

Crassicornes Guenée, 1875. Stat. sc. d'Eure-et-Loir. Lép., p. 57; legião, incluindo Globulosae, Verticillatae.

Globulosae Guenée, 1875. Stat. sc. d'Eure-et-Loir. Lép., p. 57; tribo, incluindo Procridae.

Verticillatae Guenée, 1875. Stat. sc. d'Eure-et-Loir. Lép., p. 59; tribo, incluindo Zygaenidae.

Plicatulae Guenée, 1875. Stat. sc. d'Eure-et-Loir. Lép., p. 62; legião, incluindo Lichenivora, Plantivorae.

Lichenivorae Guenée, 1875. Stat. sc. d'Eure-et-Loir. Lép., p. 62; tribo, incluindo Nolidae, Lithosidae.

Plantivorae Guenée, 1875. Stat. sc. d'Eure-et-Loir. Lép., p. 67; tribo, incluindo Emydae, Euchelidae, Callimorphidae.

Plumicornes Guenée, 1875. Stat. sc. d'Eure-et-Loir. Lép., p. 70; legião, incluindo Hirsutae, Verrucosae, Pannosae.

Hirsutae Guenée, 1875. Stat. sc. d'Eure-et-Loir. Lép., p. 70; tribo, incluindo Chelonidae.

Verrucosae Guenée, 1875. Stat. sc. d’Eure-et-Loir. Lép., p. 77; tribo, incluindo Liparidae.

Pannosae Guenée, 1875. Stat. sc. d'Eure-et-Loir. Lép., p. 79; tribo, incluindo Bombycidae.

Pecticornes Guenée, 1875. Stat. sc. d'Eure-et-Loir. Lép., p. 85; legião, incluindo Nude, Stellatae, Cuspidatae.

Nude Guenée, 1875. Stat. sc. d'Eure-et-Loir. Lép., p. 85; tribo, incluindo Endromidae.

Stellatae Guenée, 1875. Stat. sc. d'Eure-et-Loir. Lép., p. 86; tribo, incluindo Saturnidae.

Cuspidatae Guenée, 1875. Stat. sc. d'Eure-et-Loir. Lép., p. 87; tribo, incluindo Drepanulidae, Cilicidae.

Phalaenidae Guenée, 1875. Stat. sc. d'Eure-et-Loir. Lép., p. 90; legião, incluindo Fimbriatae, Geometrae.

Fimbriatae Guenée, 1875. Stat. sc. d'Eure-et-Loir. Lép., p. 90; tribo, incluindo Aventidae.

Tortriciformes Guenée, 1875. Stat. sc. d'Eure-et-Loir. Lép., p. 151; tribo, incluindo Cymbidae.- Lameere, 1936. Bull. \& Ann. Soc. ent. Belg. 76: 410, 413; grupo, incluindo Tortricoïdes, Psychoïdes.- Lameere, 1938. Rec. Inst. zool. Torley-Rousseau 5: 194, 211.

Erectae Guenée, 1875. Stat. sc. d'Eure-et-Loir. Lép., p. 153; tribo, incluindo Pygaeridae, Harpyidae, Dicranuridae, Notodontidae.

Deltoidae [sic]; Guenée, 1875. Stat. sc. d'Eure-et-Loir. Lép., p. 240; legião, incluindo Hypenidae, Rivulidae, Herminidae, Boletobidae.

Pyralidae [sic]; Guenée, 1875. Stat. sc. d'Eure-et-Loir. Lép., p. 246; legião, incluindo Squamosae, Pulverulentae, Heterogenidae, Luridae.

Heterogenidae Guenée, 1875. Stat. sc. d'Eure-et-Loir. Lép., p.
250; tribo, incluindo Sarrothripidae.

Radiati Guenée, 1875. Stat. sc. d'Eure-et-Loir. Lép., p. 265; syn.: Microlepidoptera; divisão, incluindo Elongatae.

Elongatae Guenée, 1875. Stat. sc. d'Eure-et-Loir. Lép., p. 265; legião, incluindo Depressae, Inclinatae, Involutae.

Depressae Guenée, 1875. Stat. sc. d'Eure-et-Loir. Lép., p. 265; tribo, incluindo Stenopterydae, Scoparidae.

Inclinatae Guenée, 1875. Stat. sc. d'Eure-et-Loir. Lép., p. 268; tribo, incluindo Crambidae, Chelonidae.

Crambidae Guenée, 1875. Stat. sc. d'Eure-et-Loir. Lép., p. 268; subtribo, incluindo gêneros.

Chelonidae Guenée, 1875. Stat. sc. d'Eure-et-Loir. Lép., p. 272; subtribo, incluindo Scirpophagidae, Schoenobidae.

Involutae Guenée, 1875 (nec Boisduval, 1832 = Hesperioidea). Stat. sc. d'Eure-et-Loir. Lép., p. 274; tribo, incluindo Galleridae e a subtribo Phycidae.

Phycidae Guenée, 1875. Stat. sc. d'Eure-et-Loir. Lép., p. 277; subtribo, incluindo gêneros.

Blastérocères Bar, 1878. Ann. Soc. ent. France (5)8: 11; syn.: Ephemerina, Rhopalocera.

Variables Bar, 1878. Ann. Soc. ent. France (5)8: 12; seção, incluindo Érycinides e Hespérides.- Haase, 1891. Dtsch. ent. Ztschr. "Iris" 4: 5.

Endophytes Bar, 1878. Ann. Soc. ent. France (5)8: 12; seção, incluindo Castnides.- Haase, 1891. Dtsch. ent. Ztschr. "Iris" 4: 5 .

Inclinées Bar, 1878. Ann. Soc. ent. France (5)8: 12; seção, incluindo Castnides.- Haase, 1891. Dtsch. ent. Ztschr. "Iris" 4: 5 .

Perpendiculaires Bar, 1878. Ann. Soc. ent. France (5)8: 12; seção, incluindo Satyrides, Brassolides ou Pavonides, Morphides, Apaturides, Nymphalides, Acraeides, Héliconides, Danaïdes, Méchanitides, Leptalides, Piérides, Papilionides, Lycaenides.Haase, 1891. Dtsch. ent. Ztschr. "Iris" 4: 5.

Succinctes [sic]; Bar, 1878. Ann. Soc. ent. France (5)8: 12; seção, incluindo Piérides, Papilionides, Lycaenides.- Haase, 1891. Dtsch. ent. Ztschr. "Iris" 4: 5.

Suspendues Bar, 1878. Ann. Soc. ent. France (5)8: 12; seção, incluindo Satyrides, Brassolides ou Pavonides, Morphides, Apaturides, Nymphalides, Acraeides, Héliconides, Danaïdes, Méchanitides, Leptalides.- Haase, 1891. Dtsch. ent. Ztschr. "Iris" 4: 5.

Hetéropodes [= Heteropoda] Bar, 1878. Ann. Soc. ent. France (5)8: 13; seção, incluindo Érycinides, Hespérides e Castnides.- Haase, 1891. Dtsch. ent. Ztsch. "Iris" 4: 5.

Makrolepidoptera [sic]; Plötz, 1886. Mitt. naturw. Ver. NeuVorpomm. u. Rügen 17: 485, 488.

Mikrolepidoptera [sic]; Plötz, 1886. Mitt. naturw. Ver. NeuVorpomm. u. Rügen 17: 487, 578.

Danaiform Doherty, 1886. Jour. Asiatic Soc. Bengal 55(2): 108; grupo, incluindo Danaidae, Acraeidae, Heliconiadae.

Revista Brasileira de Zoologia 23 (1): 1-41, março 2006 
Satyriform Doherty, 1886. Jour. Asiatic Soc. Bengal 55(2): 108; grupo, incluindo Satyridae, Elymniadae, Morphidae, Brassolidae.

Nymphaliform Doherty, 1886. Jour. Asiatic Soc. Bengal 55(2): 108; grupo, incluindo Nymphalidae.

Apaturiform Doherty, 1886. Jour. Asiatic Soc. Bengal 55(2): 108; grupo, incluindo Apaturidae, Eurytelidae.

Lycaeniform Doherty, 1886. Jour. Asiatic Soc. Bengal 55(2): 108; grupo, incluindo Lycaenidae.

Pieriform Doherty, 1886. Jour. Asiatic Soc. Bengal 55(2): 108; grupo, incluindo Pieridae, Libytheidae.

Heteropoda [sic]; Doherty, 1886. Jour. Asiatic Soc. Bengal. 55(2): 108, 110, 126.- Haase, 1891. Dtsch. ent. Ztschr. "Iris" 4: 12.

Uranides Guenée, 1887, in Boisduval \& Guenée. Hist. nat. Ins. Sp. gén. Lép. 9, p. 1; grupo, incluindo Cydimonidae, Uranidae, Nyctalemonidae, Sematuridae.

Paleohesperidae Grote, 1889. Can. Ent. 21: 205, 208; família, incluindo Papilionidae, em oposição a Hesperiadae.

Sorbentia Haeckel, 1889. Natürl. Schöpfungs-Gesch. $8^{\text {a }}$ ed., p. 588, 593 [= Lepidoptera]; Handlirsch (1925), na sinonímia de Lepidoptera, atribui este nome à Haeckel, 1866,

Homoiopoda Haase, 1891. Dtsch. ent. Ztschr. "Iris" 4: 12; syn.: Hexapoda; série evolutiva, incluindo Hesperiidae, Papilionidae, Pieridae.

Amblyopoda Haase, 1891. Dtsch. ent. Ztschr. "Iris" 4: 12; syn.: Tetrapoda; sem status, incluindo Nymphalida.

Succincti [sic]; Haase, 1891. Dtsch. ent. Ztschr. "Iris" 4: 3, 6. Danaiformes [sic]; Haase, 1891. Dtsch. ent. Ztschr. "Iris" 4: 25; seção, incluindo Danaiden, Acraeiden, Heliconiden.

Satyriformes [sic]; Haase, 1891. Dtsch. ent. Ztschr. "Iris" 4: 25; seção, incluindo Satyriden, Morphiden, Brassoliden.

Nymphaliformes [sic]; Haase, 1891. Dtsch. ent. Ztschr. "Iris" 4: 25; seção, incluindo Nymphaliden.

Apaturiformes [sic]; Haase, 1891. Dtsch. ent. Ztschr. "Iris" 4: 25; seção, incluindo Apaturiden, Euryteliden.

Lycaeniformes [sic]; Haase, 1891. Dtsch. ent. Ztschr. "Iris" 4: 25; seção, incluindo Lycaenidae.

Pieriformes [sic]; Haase, 1891. Dtsch. ent. Ztschr. "Iris" 4: 25; seção, incluindo Pieriden, Libytheiden.

Danaomorpha Haase, 1891. Dtsch. ent. Ztschr. "Iris" 4: 29; família, incluindo Danainae, Neotropinae, Palaeotropinae.

Satyromorpha Haase, 1891. Dtsch. ent. Ztschr. "Iris" 4: 29; família, incluindo Brassolinae, Satyrinae, Morphinae.

Acraeomorpha Haase, 1891. Dtsch. ent. Ztschr. "Iris" 4: 31; família, incluindo Nymphalinae, Heliconiinae, Acraeinae.

Frenatae J. H. Comstock, 1892. Proc. Amer. Ass. Sc. 61: 200; subordem oposta a Jugatae, incluindo as demais famílias.- J. H. Comstock, 1892. Zoe 3(1): 86.- J. H. Comstock, 1893, in Wilder. The Wilder Quarter-Century Book, p. 97, 100.- Dyar, 1894. Ann. N. Y. Acad. Sc. 8: 202, 205.- Packard, 1895. Mem. Nat. Ac. Sc. 7: 57.- Grote, 1895. Mitt. Roemer-Mus., Hildesheim, 1: [2].- Dyar, 1895. Jour. N. Y. Ent. Soc. 3: 17.-
Dyar, 1895. Amer. Nat. 29: 1066, 1068, 1071.- J. H. Comstock \& A. B. Comstock, 1895. Man. Study Ins., p. 204, 216.- Dyar, 1895. Trans. N. Y. Ac. Sc. 14: 51, 54, 61.- Bodine, 1896. Trans. Amer. Ent. Soc. 23: 8, 23, 24, 27, 28, 29, 30, 34, 45.- Grote, 1896. Mitt. Roemer-Mus., Hildesheim, 7: 2.- Tutt, 1897. Ent. Rec \& Jour. Var. 9: 194, 195.- Grote, 1897. Mitt. RoemerMus., Hildesheim, 8: 6.- Karsch, 1898. Ent. Nachr. 24: 297.J. H. Comstock \& A. B. Comstock, 1904. Man. Study Ins.4 ${ }^{\mathrm{a}}$ ed., p. 201, 202, 203, 204, 216.- Bode, 1907. Mitt. RoemerMus., Hildesheim, 22: 7.- Handlirsch, 1907. Die fossilen Ins. 2, p. 1257, 1292.- Fracker, 1915. Illinois Biol. Monogr. 2: 28, 58.- Heymons, 1915, in zur Srassen. Brehms Tierleben. $4^{\mathrm{a}}$ ed., p. XVII, 217.- J. H. Comstock, 1918. Wings Ins., p. 318, 325, 326, 330.- Krausse \& Wolff, 1919. Arch. Natg. 86A(3): 165.- Forbes, 1923. Mem. Cornell. Univ. Agric. Exp. Stat. 68: 34, 70.- Handlirsch, 1925, in Schröder. Handb. Ent. 3, p. 870; syn.: Neolepidoptera, Haustellata, Anisoneuria, Heteroneura, Glossata.- Wolff \& Krausse, 1930. Wirbell. Tiere, p. 199.- Börner, 1939. Verh. VII intern. Kongr. Ent. 2, p. 1372, 1385.- J. H. Comstock, 1940. Introd. Ent. 9a ed., p. 582, 597.- Le Marchand, 1945. Rev. franç. Lép. 10: 139.Kiriakoff, 1946. Lambillionea 46: 55, 114.- Hennig, 1953. Beitr. Ent. 3, Sonderh., p. 47.- Richards \& Davies, 1957, in Imms. Gen. Textb. Ent., p. 533.- Friese, 1969. Bericht 10. Wandervers. Deutscher Ent., Tagungsb. 80(2): 205, 212.Razowski, 1974. Acta zool. cracov. 19: 8.-Scoble, 1992. Lep., p. 190, 191.

Jugatae J. H. Comstock, 1892. Proc. Amer. Ass. Sc. 61: 200; subordem, incluindo Hepialidae, Micropterygidae.- J. H. Comstock, 1892. Zoe 3(1): 86.- J. H. Comstock, 1893, in Wilder. The Wilder Quarter-Century Book, p. 97, 98.- Dyar, 1894. Ann. N. Y. Ac. Sc. 8: 202, 203, 204.- J. H. Comstock \& A. B. Comstock, 1895. Man. Study Ins., p. 204, 207, 214.Packard, 1895. Mem. Nat. Ac. Sc. 70:57.- Dyar, 1895. Jour. N. Y. Ent. Soc. 3: 17.- Dyar, 1895. Amer. Nat. 29: 1066, 1068, 1069.- Dyar, 1895. Trans. N. Y. Ac. Sc. 14: 51.- Bodine, 1896. Trans. Amer. Ent. Soc. 23: 8, 9, 23, 24, 27, 28, 45.Tutt, 1897. Ent. Rec. \& Jour. Var. 9: 194, 195.- Karsch, 1898. Ent. Nachr. 24 297.- Chapman, 1899. Ent. Rec. \& Jour. Var. 11: 122, 124.- J. H. Comstock \& A. B. Comstock, 1904. Man. Study Ins. $4^{\text {a }}$ ed., p. 201, 204, 214.- Handlirsch, 1907. Die fossilen Ins. 2, p. 1257, 1292.- Fracker, 1915. Illinois Biol. Monogr. 2: 23, 27, 57.- Heymons, 1915, in zur Srassen. Brehms Tierleben. $4^{\text {a }}$ ed., p. XVIII, 216.- J. H. Comstock, 1918. Wings Ins., p. 325, 326, 327.- Krausse \& Wolff, 1919. Arch. Natg. 86A(3): 165.- Forbes, 1923. Mem. Cornell Univ. Agric. Exp. Stat. 68: 27, 34, 62.- Handlirsch, 1925, in Schröder. Handb. Ent. 3, p. 867; syn.: Stemmatoncopoda, Isoneuria, Homoneura, Micropterygina, Laciniata, Haustellata, Palaeolepidoptera, Neolepidoptera, Micropterygoidea, Laciniata, Glossata, Archilepidoptera.- Wolff \& Krausse, 1930. Wirbell. Tiere, p. 199.- Börner, 1939. Verh. VII intern. Kongr. Ent. 2, p. 1372, 1385.- J. H. Comstock, 1940. Introd. Ent. 9a ed.,

Revista Brasileira de Zoologia 23 (1): 1-41, março 2006 
p. 581, 592.- Le Marchand, 1945. Rev. franç. Lép. 10: 139.Kiriakoff, 1946. Lambillionea 46: 55, 114.- Hennig, 1953. Beitr. Ent. 3, Sonderh., p. 47.- Richards \& Davies, 1957, in Imms. Gen. Textb. Ent., p. 533.- Friese, 1969. Bericht 10. Wandervers. Deutscher Ent., Tagungsb. 80(2): 205, 207, 212.- Scoble, 1989, in Emmet \& Head. Moths and Butt. Great Brit and Ireland 7, p. 17, 18.- Scoble, 1992. Lep., p. 190, 191.

Macrojugatae J. H. Comstock, 1893, in Wilder. The Wilder Quarter-Century Book, p. 97, 99; sem status, incluindo Hepialidae.- J. H. Comstock \& A. B. Comstock, 1895. Man. Study Ins., p. 204.

Microjugatae J. H. Comstock, 1893, in Wilder. The Wilder Quarter-Century Book, p. 97, 99; sem status, incluindo Micropterygidae.- J. H. Comstock \& A. B. Comstock, 1895. Man. Study Ins., p. 204.

Microfrenatae J. H. Comstock, 1893, in Wilder. The Wilder Quarter-Century Book, p. 97, 100; sem status, incluindo as superfamílias Tineina, Tortricina, Pyralidina.- J. H. Comstock \& A. B. Comstock, 1895. Man. Study Ins., p. 205.- Packard, 1895. Mem. Nat. Ac. Sc. 7: 57.- Bodine, 1896. Trans. Amer. Ent. Soc. 23: 19, 20, 34, 35, 37.- J. H. Comstock \& A. B. Comstock, 1904. Man. Study Ins. $4^{\text {a }}$ ed., p. 205.- Heymons, 1915, in zur Srassen. Brehms Tierleben. $4^{\text {a }}$ ed., p. XVIII, 217; incluindo "micros", Castniidae e Hesperiidae.- J. H. Comstock, 1940. Introd. Ent. 9a ed., p. 582, 589, 610.- Kiriakoff, 1946. Lambillionea 46: 111; syn.: Stemmatoncopoda.

Macrofrenatae J. H. Comstock, 1893, in Wilder. The Wilder Quarter-Century Book, p. 97, 101; sem status e oposto a Microfrenatae, incluindo as demais superfamílias.- J. H. Comstock \& A. B. Comstock, 1895. Man. Study Ins., p. 205, 206.- Packard, 1895. Mem. Nat. Ac. Sc. 7: 57.- Bodine, 1896. Trans. Amer. Ent. Soc. 23: 23, 34, 37.- J. H. Comstock \& A. B. Comstock, 1905. Man. Study Ins., p. 205.- 1915, in zur Strassen. Brehms Tierleben. $4^{\text {a }}$ ed., p. XIX, 246; incluindo Opistoneura e Enantioneura.- Handlirsch, 1925, in Schröder. Handb. Ent. 3, p. 910; syn.: Heterocera, Macrolepidoptera, Anisoneuria, Harmoncopoda, Stemmatoncopoda, Macrofrenatae, Haustellata, Neolepidoptera, Macroptera.- J. H. Comstock, 1940. Introd. Ent. 9a ed., p. 583, 655.- Kiriakoff, 1946. Lambillionea 46: 111, 112; syn.: Harmoncopoda.

Incompletae Chapman, 1893. Trans. ent. Soc. London, p. 100, 101, 103, 106, 107, 109, 111, 113-115, 117, 118; syn.: Micros; grupo, incluindo Pterophorina, Lithocolletidae, Gracilariidae, Tineidae, Psychidae, Sesiidae, Tortricina, Cossus, Exapate, Simaethis, Castnia, Micropterygidae, Cochliopodidae, Zygaenidae, Lyonetia, Cemiostoma, Bedellia, Eriocephala, Zeuzera, Hepialus, Tischeria, Adelidae, Nepticulidae.- Pachard, 1895. Zool. Anz. 18: 234, 236.- Chapman, 1896. Trans. ent. Soc. London, p. 571, 572.- Tutt, 1897. Ent. Rec. \& Jour. Var. 9: 194, 195.- Tutt, 1899. Brit. Lep. 1, p. 107.- Kiriakoff, 1946. Lambillionea 46: 54.- Razowski, 1974. Acta zool. cracov. 19: 8 .
Obtectae Chapman, 1893. Trans. ent. Soc. London, p. 100, 103, 104, 106-107, 109, 110, 113, 115-118; syn.: Macros; grupo, incluindo Sphinges, Bombyces, Nolidae, Nycteolidae, Noctuina, Geometrae, Pyrales, Phycidae, Eudoridae, Crambidae, Gelechiidae, Plutellidae, Oecophoridae, Epigraphiidae, Alucitidae, Hyponymeutidae, Argyresthidae, Coleophoridae, Perittia, Elachistidae.- Packard, 1895. Zool. Anz. 18: 234, 236.Chapman, 1896. Trans. ent. Soc. London, p. 572-574.- Tutt, 1897. Ent. Rec. \& Jour. Var. 9: 194, 195.- Chapman, 1899. Ent. Rec. \& Jour. Var. 11:122.- Tutt, 1899. Brit. Lep. 1, p. 107.- Kiriakoff, 1946. Lambillionea 46: 54.- Razowski, 1974. Acta zool. cracov. 19: 8.

Micros Chapman, 1893. Trans. ent. Soc. London, p. 100, 111; na sinonímia de Incompletae.

Macros Chapman, 1893. Trans. ent. Soc. London, p. 100, 110, 114; na sinonímia de Obtectae.

Pterophori Chapman, 1893. Trans. ent. Soc. London, p. 104, 110; sem status, sem indicação de grupo.

Nepticulae Chapman, 1893. Trans. ent. Soc. London, p. 105; sem status, sem indicação de grupo.

Macroheterocera Chapman, 1893. Trans. ent. Soc. London, p. 106, 117; sem status, sem indicação de grupo.- Chapman, 1895. Ent. Rec. \& Jour. Var. 6: 103, 104.- Chapman, 1896. Trans. ent. Soc. London, p. 570, 577, 578, 579, 582.Fracker, 1915. Illinois Biol. Monogr. 2: 94.

Tortricoids Chapman, 1893. Trans. ent. Soc. London, p. 111; grupo, sem indicar taxa incluidos.

Tineoids Chapman, 1893. Trans. ent. Soc. London, p. 111; grupo, sem indicar taxa incluidos.- Packard, 1895. Zool. Anz. 18: 235; syn.: Stenopterygia; seção de Neolepidoptera.- Packard, 1895. Mem. Nat. Ac. Sc. 7: 62 [inclue os grupos com asas estreitas].

Jugateae [sic]; Dyar, 1895. Trans. N. Y. Ac. Sc. 14: 50.

Macro-Heterocera [sic]; Dyar, 1895. Trans. N. Y. Ac. Sc. 14: 50.

Saturnina Dyar, 1895. Trans. N. Y. Ac. Sc. 14: 53, 54, 62; syn.: Bombycina; superfamília, incluindo Citheroniidae, Hemileucidae, Saturniidae, Agliidae, Bombycidae.

Laciniata Packard, VI-1895. Zool. Anz. 18: 229, 233, 236; syn.: Protolepidoptera; divisão ou subordem, incluindo Eriocephalidae [= Micropterigidae].- Packard, VII-1895. Amer. Nat. 29: 636, 637; syn.: Protolepidoptera; subordem, incluindo Eriocephalidae.- Packard, 1895. Mem. Nat. Ac. Sc. 7: 58; syn.: Protolepidoptera.- Tutt, 1897. Ent. Rec. \& Jour. Var. 9: 196; syn.: Protolepidoptera.- Karsch, 1898. Ent. Nachr. 24: 297.Tutt, 1899. Brit. Lep. 1, p. 106; syn.: Protolepidoptera.Chapman, 1916. Trans. ent. Soc. London, p. 310.- Börner, 1920, in Brohmer. Fauna Deutschland. $2^{\mathrm{a}}$ ed., p. 341; syn.: Frenatae, Protolepidoptera, Archilepidoptera, Jugatae.- Börner, 1939. Verh. VII. intern. Kongr. Ent. 2, p. 1372, 1389.Kiriakoff, 1946. Lambillionea 46: 24, 112; syn.: Protolepidoptera.- Kiriakoff, 1948. Biol. Jaarboek, Antwerpen, 15, p. 131.- Friese, 1969. Bericht 10. Wandervers. Deutscher 
Ent., Tagungsb. 80(2): 205.- Razowski, 1974. Acta zool. cracov. 19: 9; syn.: Protolepidoptera.- Speidel, 1977. Atalanta 8: 119, 120.- Kuznetzov \& Stekolnikov, 1978. Ent. Obozr. 57: 881, 882, 887; syn.: Protolepidoptera, Zeugloptera.Kuznetzov \& Stekolnikov, 1979. Rev. Ent. 57: 609, 610, 613; syn.: Protolepidoptera, Zeugloptera.

Protolepidoptera Packard, VI-1895. Zool. Anz. 18: 229; na sininímia de Laciniata.- Packard, VII-1895. Amer. Nat. 29: 637.- Dyar, 1895. Amer. Nat. 29: 1069.- Tutt, 1897. Ent. Rec. \& Jour. Var. 9: 194.- Karsch, 1898. Ent. Nachr. 24: 297.- Chapman, 1916. Trans. ent. Soc. London, p. 310.Kiriakoff, 1948. Biol. Jaarboek, Antwerpen, 15, p. 131.Küppers \& Speidel, 1980. Atalanta 11: 58.

Palaeolepidoptera Packard, VI-1895. Zool. Anz. 18: 234, 236; syn.: Liberae; série de superfamílias subordinadas à Haustellata, incluindo Micropterygidae [sic] [= Eriocraniidae].Tutt, 1899. Brit. Lep. 1, p. 106, 114.- Kiriakoff, 1948. Biol. Jaarboek, Antwerpen, 15, p. 131.- Razowski, 1974. Acta zool. cracov. 19: 9.

Liberae Packard, VI-1895. Zool. Anz. 18: 234, 236; na sinonímia de Palaeolepidoptera.- Packard, VII-1895. Amer. Nat. 29: 644; na sininímia de Paleolepidoptera [sic].

Neolepidoptera Packard, VI-1895. Zool. Anz. 18: 234, 235, 236; série de superfamílias subordinadas à Haustellata [tudo menos Zeugloptera].- Packard, VII-1895. Amer. Nat. 29: 645.Packard, 1895. Mem. Nat. Ac. Sc. 7: 61.- Dyar, 1895. Amer. Nat. 29: 1067.- Chapman, 1899. Ent. Rec. \& Jour. Var. 11: 122.- Tutt, 1899. Brit. Lep. 1, p. 106, 114.- Kiriakoff, 1948. Biol. Jaarboek, Antwerpen, 15, p. 131.- Hennig, 1953. Beitr. Ent. 3, Sonderh., p. 47.- Kiriakoff, 1955. Bull \& Ann. Soc. Ent. Belg. 91: 155, 158.- Razowski, 1974. Acta zool. cracov. 19: 9, 10.- Davis \& Nielsen, 1980. Steenstrupia 6: 273 (= Exoporia+Heteroneura; syn.: Frenata).- Küppers \& Speidel, 1980. Atalanta 11: 58-60, 63.- Kristensen \& Nielsen, 1981, in Cederholm. Ent. scand., Suppl. 15: 301, 302, 303.- Minet, 1984. Nouv. Rev. Ent. (n. s.) 1: 141, 143.- Kristensen, 1984. Steenstrupia 10: 163, 186.- Nielsen, 1985. Jour. Res. Lep., Suppl. 1: 3.- Minet, 1986. Alexanor 14: 291 (como Néolépidoptères).- Nielsen, 1989, in Fernholm; Bremer \& Jörnvall (Ed.). Hierarchy Life, p. 282, 284, 285, 291.- Scoble, 1989, in Emmet \& Head. Moths and Butt. Great Brit. and Ireland 7, p. 15, 17, 18, 22.- Nielsen \& Common, 1991, in CSIRO. Ins. Australia, p. 833, 837.- Scoble, 1995. Lep., p. 188.- Poole, 1996, in Poole \& Gentili. Nom. Ins. Nearct. 3, p. 612.- Nielsen \& Kristensen, 1996. Invert. Tax. 10: 1200, 1201, 1251, 1253, 1261, 1270, 1285, 1291, 1294.- Kristensen \& Skalski, 1999, in Kristensen (Ed.), Lep. 1, p. 10, 11, in Fischer (Ed.). Handb. Zool. 4.- Carter \& Kristensen, 1999, in Kristensen (Ed.), Lep. 1, p. 28, in Fischer (Ed.). Handb. Zool. 4.- Kristensen, 1999, in Kristensen (Ed.), Lep. 1, p. 56, in Fischer (Ed.). Handb. Zool. 4.- Heppner, 2003. Lep. Florida 1, p. 71, 91, 94, 231; subcoorte.

Stenopterygia Packard, VI-1895. Zool. Anz. 18: 235; na sinonímia de Tineoids.

Platylepidoptera Packard, VI-1895. Zool. Anz. 18: 235; na sinonímia de Macrolepidoptera.- Packard, 1895. Mem. Nat. Ac. Sc. 7: 62; sinonímia de Macrolepidoptera.

Paleolepidoptera [sic]; Packard, VII-1895. Amer. Nat. 29: 644.Packard, 1895. Mem. Nat. Ac. Sc. 7: 61, 62 (part.).- Dyar, 1895. Amer. Nat. 29: 1069.- Küppers \& Speidel, 1980. Atalanta 11: 58, 59, 63; syn.: Palaeolepidoptera, Dacnonypha, Hoplostomatoptera, Dachnonypha [sic], Eriocraniomorpha.

Caridrinina Meyrick, 1895. Handb. brit. Lep., p. 21, 22; grupo, incluindo Ocneriadae, Caradrinidae, Plusiadae, Arctiadae.Meyrick, 1928. Rev. Handb. brit. Lep., p. 29, 30.- Le Marchand, 1945. Rev. franç. Lép. 10: 110.- Turner, 1947. Proc. Linn. Soc. N. S. Wales 71: 304, 305.- Common, 1975. Ann. Rev. Ent. 20: 183.

Anthrocerina Dyar, 1895. Trans. N. Y. Ac. Sc. 14: 53, 54; superfamília, incluindo Pterophoridae, Anthroceridae, Pyromorphidae, Megalopgidae, Eucleidae.- Dyar, 1895. Ent. News 6: 39.- Dyar, 1895. Jour. N. Y. Ent. Soc. 3: 25.- Tutt, 1899. Brit. Lep. 1, p. 107, 109.

Saturnia Dyar, 1895. Jour. N. Y. Ent. Soc. 3: 26; syn.: Bombyces; sem status, sem indicação de grupo.

Frenulum-conservers Bodine, 1896. Trans. Amer. Ent. Soc. 23: 37, 38, 39; subdivisão de Frenatae, incluindo as famílias com preservaram o frênulo.

Frenulum-losers Bodine, 1896. Trans. Amer. Ent. Soc. 23: 37, 41; subdivisão de Frenatae, incluindo as famílias que perderam o frênulo, sendo substituido por uma expansão do ângulo umeral da asa posterior.

Micro-Heterocera Tutt, 1896. Brit. Butt., p. 118; sem status, sem definição.

Parnassi-Papilionidae Grote, 1897. Mitt. Roemer-Mus., Hildesheim, 8: 8; sem status, inluindo Parnassidae, Papilionidae.

Pieri-Hesperiidae Grote, 1897. Mitt. Roemer-Mus., Hildesheim, 8: 8; sem status, incluindo Pieridae, Nymphalidae, Agapetidae, Limnadidae, Libytheidae, Nemeobiidae, Riodinidae, Lycaenidae, Hesperiidae, Megathymidae.

Sphingo-Limacodid Tutt, 1897. Ent. Rec. \& Jour. Var. 9: 196; estirpe, incluindo Cochliopodides, Zygaenides, Lasiocampides, Geometrides, Endromides, Saturniides, Sphingides.

Noctuo-Hepialid Tutt, 1897. Ent. Rec. \& Jour. Var. 9: 196; estirpe, incluindo Hepialides, Zeuzerides, Cossides, Tortricides, Nyctaeolides, Nolides, Notodontides, Liparides, Arctiides, Noctuides, Hesperides, Papilionides.

Pyralo-Micropterygid Tutt, 1897. Ent. Rec. \& Jour. Var. 9: 196; estirpe, incluindo Micropterygides, Adelides, Tineides, Sesiides (?), Psychides (?), Coleophorides, Crambides, Pyralides, Platypterygides.

Isoneuria Karsch, 1898. Ent. Nachr. 24: 299, 302; divisão, incluindo Hepialidae, Eriocephalidae e Micropterygidae; oposto a Anisoneuria.- Kiriakoff, 1946. Lambillionea 46: 24-26.Agenjo, 1946. Graellsia 4(3): [7].- Kiriakoff, 1948. Biol.

Revista Brasileira de Zoologia 23 (1): 1-41, março 2006 
Jaarboek, Antwerpen, 15, p. 128.- Friese, 1969. Bericht 10. Wandervers. Deutscher Ent., Tagungsb. 80(2): 205, 207.Razowski, 1974. Acta zool. cracov. 19: 9.

Anisoneuria Karsch, 1898. Ent. Nachr. 24: 299, 302; divisão, oposto a Isoneuria, incluindo as demais famílias.- Kiriakoff, 1946. Lambillionea 46: 24, 62.- Agenjo, 1946. Graellsia 4(3): [7].- Kiriakoff, 1948. Biol. Jaarboek, Antwerpen, 15, p. 128.- Friese, 1969. Bericht 10. Wandervers. Deutscher Ent., Tagungsb. 80(2): 205, 207, 218; syn.: Heteroneura.Razowski, 1974. Acta zool. cracov. 19: 9.

Harmoncopoda Karsch, 1898. Ent. Nachr. 24: 302, 303; subordem, incluindo borboletas e mariposas "superiores".Börner, 1904. Zool. Anz. 27: 533.- Heymons, 1915, in zur Strassen. Brehms Tierleben. $4^{\text {a }}$ ed., p. XIX, 246.- Börner, 1939. Verh. VII intern. Kongr. Ent., Berlin, 2, p. 1372, 1377.- Kiriakoff, 1946. Lambillionea 46: 53.- Kiriakoff, 1946. Biol. Jaarboek, Antwerpen, 13, p. 290.- Kiriakoff, 1948. Biol. Jaarboek, Antwerpen, 15, p. 137, 138, 141.Kiriakoff, 1952. Ann. Soc. r. zool. Belg. 83: 98.- Kiriakoff, 1955. Bull. \& Ann. Soc. ent. Belg. 91: 156-158.- Friese, 1969. Bericht 10. Wandervers. Deutscher Ent., Tagungsb. 80(2): 205.- Razowski, 1974. Acta zool. cracov. 19: 9, 10, 15.Scoble, 1989, in Emmet \& Head. Moths and Butt. Great Brit. and Ireland 7, p. 17, 18.- Scoble, 1992. Lep., p. 190, 191.- Gómez B., 1978. Shilap, Revta lepid. 6: 246.

Stemmatoncopoda Karsch, 1898. Ent. Nachr. 24: 302, 303; subordem, incluindo mariposas "primitivas" e Hesperiidae.Börner, 1904. Zool. Anz. 27: 533.- Heymons, 1915, in zur Srassen. Brehms Tierleben. $4^{\text {a }}$ ed., p. XVIII, 217.- Börner, 1939. Verh. VII intern. Kongr. Ent., Berlin, 2, p. 1372, 1377.- Kiriakoff, 1946. Lambillionea 46: 53, 112.- Kiriakoff, 1946. Biol. Jaarboek, Antwerpen, 13, p. 290.- Kiriakoff, 1948. Biol. Jaarboek, Antwerpen, 15, p. 137, 138, 140.Kiriakoff, 1952. Ann. Soc. r. zool. Belg. 83: 98.- Kiriakoff, 1955. Bull. \& Ann. Soc. ent. Belg. 91: 156-158.- Friese, 1969. Bericht 10. Wandervers. Deutscher Ent., Tagungsb. 80(2): 205.- Razowski, 1974. Acta zool. cracov. 19: 9, 10, 15.Gómez B., 1978. Shilap, Revta lepid. 6: 246.- Scoble, 1989, in Emmet \& Head. Moths and Butt. Great Brit. and Ireland 7, p. 17, 18.- Scoble, 1992. Lep., p. 190, 191.

Sphingo-Micropterygides Tutt, 1899. Brit. Lep. 1, p. 114, 115; estirpe, incluindo Micropterygides, Nepticulides, Eucleides, Megalopygides, Heterogynides, Antherocerides, Psychides, Pterophorides.

Tineides aculeatae Spuler, 1910. Schmett. Europas 2, p. 464; superfamília, incluindo Incurvarioidea, Tischeriidae, Heliozelidae.- Le Marchand, 1945. Rev. franç. Lép. 10: 104, 105, 107.

Archilepidoptera Spuler, 1910. Schmett. Europas 2, p. 482; subordem, incluindo Eriocraniidae, Micropterygidae e Hepiolidae [sic].- Börner, 1939. Verh. VII intern. Kongr. Ent., Berlin, 2, p. 1372.- Razowski, 1974. Acta zool. cracov. 19: 9.

Frenata [sic]; Kuznetzov, 1915. Fauna Russ., p. 10.- Tillyard,
1918. Proc. Linn. Soc. N. S. Wales 43: 296.- Kiriakoff, 1946. Lambillionea 46: 23, 24, 62.- Kiriakoff, 1946. Zool. Jaarboek, Antwerpen, 13, p. 290.- Agenjo, 1946. Graellsia 4(3): [7].- Kiriakoff, 1948. Biol. Jaarboek, Antwerpen, 15, p. 128.- Hennig, 1953. Beitr. Ent. 3, Sonderh., p. 47.Kiriakoff, 1955. Bull. \& Ann. Soc. ent. Belg. 91: 155-158.Kuznetzov, 1967. Fauna Russia., reimpr., p. 8, 14.- Razowski, 1974. Acta zool. cracov. 19: 9, 10; syn.: Frenatae.- Küppers \& Speidel, 1980. Atalanta 11: 61-63; syn.: Frenatae, Anisoneuria, Heteroneura.- Nielsen, 1989, in Fernholm; Bremer \& Jörnvall (Ed.). Hierarchy Life, p. 282.

Jugata [sic]; Kuznetzov, 1915. Fauna Russ., p. 10.- Tillyard, 1918. Proc. Linn. Soc. N. S. Wales 43: 296.- Kiriakoff, 1946. Lambillionea 46: 23, 24, 62.- Kiriakoff, 1948. Biol. Jaarboek, Antwerpen, 15, p. 128.- Kuznetzov, 1967. Fauna Russ., reimpr., p. 8, 14.- Razowski, 1974. Acta zool. cracov. 19: 8; syn.: Jugatae.

Opisthoneura Heymons, 1915, in zur Srassen. Brehms Tierleben. $4^{\text {a }}$ ed., p. XIX, 246; série de famílias, incluindo famílias de mariposas.- Kiriakoff, 1946. Lambillionea 46: 112.

Enantioneura Heymons, 1915, in zur Srassen. Brehms Tierleben. $4^{\mathrm{a}}$ ed., p. XX, 269; série de famílias, incluindo Heterocera e Rhopalocera.- Kiriakoff, 1946. Lambillionea 46: 112.

Aculeata Fracker, 1915. Illinois Biol. Monogr. 2: 58; sem status, incluindo Nepticulidae, Prodoxidae, Incurvariidae, Tischeriidae.

Non-Aculeatae Fracker, 1915. Illinois Biol. Monogr. 2: 58; sem status, incluindo os Microlepidoptera não aculeados, em oposição aos Aculeata.

Zeugloptera Chapman, 1916. Trans. ent. Soc. London, p. 310 314; subordem, incluindo Micropteryx.- Hinton, 1946. Trans. ent. Soc. London 97: 2, 3.- Kiriakoff, 1946. Lambillionea 46: 22, 23.- Kiriakoff, 1948. Biol. Jaarboek, Antwerpen, 15, p. $127,134,135,136,142 .-$ Kiriakoff, 1952. Ann. Soc. r. zool. Belg. 83: 95.- Hennig, 1953. Beitr. Ent. 3, Sonderh., p. 47.- Kiriakoff, 1955. Bull. \& Ann. Soc. ent. Belg. 91: 155-158.- Richards \& Davies, 1957, in Imms. Gen. Textb. Ent., p. 533, 534, 537.- Friese, 1969. Bericht 10. Wandervers. Deutscher Ent., Tagungsb. 80(2): 207, 208, 218; syn.: Protolepidoptera, Laciniata.- Common, 1970, in CSIRO. Ins. Australia, p. 782, 783, 784.- Brock, 1971. Jour. nat. Hist. 5: 34, 48.- Razowski, 1974. Acta zool. cracov. 19: 3, 9, 10, 12.- Dugdale, 1974. N. Zealand Jour. Zool. 1: 129, 132, 143.- Common, 1974, in CSIRO. Ins. Australia, Suppl., p. 101.- Birkit-Smith, 1974. Ent. scand. 5: 161, 173.Common, 1975. Ann. Rev. Ent. 20: 193, 199.- Speidel, 1977. Atalanta 8: 119, 120.- Gómez B., 1978. Shilap, Revta lepid. 6: 247, 247bis, fig., 251, 257.- Gómez B., 1979. Shilap, Revta lepid. 7: 169.- Kristensen \& Nielsen, 1981, in Cederholm. Ent. scand., Suppl. 15: 300, 303.- Kristensen \& Nielsen, 1983. Ztschr. zool. Syst. Evolut.-forsch. 21: 114, 115, 116, 118, 119.- Kristensen, 1984. Steenstrupia 10: 153, 169, 186.- Heppner, 1984. Atlas neotrop. Lep. 2. Checkl. 1, p. XIX.- Minet, 1984. Nouv. Rev. Ent. (n. s.) 1: 147.-

Revista Brasileira de Zoologia 23 (1): 1-41, março 2006 
Kristensen, 1985. Ent. Medd. 52: 9.- Nielsen, 1985. Jour. Res. Lep., Suppl. 1: 2, 15.- Minet, 1986. Alexanor 14: 291, 292.- Hennig, 1986. Taschenb. Zool. 3, Wirbellose II. $4^{\mathrm{a}}$ ed., p. 230.- Holloway; Bradley \& Carter, 1987. CIE Guides ins. import. man. 1. Lepidoptera, p. 17, 23, 25, 38.Nielsen, 1989, in Fernholm; Bremer \& Jörnvall (Ed.). Hierarchy Life, p. 281-284, 291.- Scoble, 1989, in Emmet \& Head. Moths and Butt. Great Brit. and Ireland 7, p. 15, 17, 19.- Nielsen \& Common, 1991, in CSIRO. Ins. Australia, p. 831, 833, 834.- Scoble, 1992. Lep., p. 188, 195.- Heppner, 1995. Atlas neotrop. Lep. 3. Checkl. 2, p. XIII.- Poole, 1996, in Poole \& Gentili. Nom. Ins. Nearct. 3, p. 612.- Heppner, 1996. Atlas neotrop. Lep. 5B. Checkl. 4B, p. XV.- Heppner, 1998. Lep. News 1998 (3): 57.- Heppner, 1998. Holarct. Lep. 5, Suppl. 1: 7, 18.- Covell, 1999. Kentucky State Nat. Pres. Comm., Sc. Tech. Ser. 6: XI, 15.- Kristensen \& Skalski, 1999, in Kristensen (Ed.), Lep. 1, p. 9, in Fischer (Ed.). Handb. Zool. 4.- Powell et al., 1999, in Kristensen (Ed.), Lep. 1, p. 405, in Fischer (Ed.). Handb. Zool. 4.- Heppner, 2003. Lep. Florida 1, p. 71; subordem.

Homoneura Tillyard, 1918. Proc. Linn. Soc. N. S. Wales 43: 297, 298, 315; subordem, incluindo Micropterygidae [sic], Prototheoridae, Hepialidae.- Kiriakoff, 1946. Lambillionea 46: 24, 26, 62.- Agenjo, 1946. Graellsia 4(3): [7].- Turner, 1947. Proc. Linn. Soc. N. S. Wales 71: 307, 337; syn.: Jugata.Warren, 1947. Entomol. 80: 216.- Kiriakoff, 1948. Biol. Jaarboek, Antwerpen, 15, p. 128.- Bourgogne, 1951, in Grassé. Traité Zool. 10(1), p. 368; syn.: Jugatae, Isoneuria, Archilepidoptera.- Hennig, 1953. Beitr. Ent. 3, Sonderh., p. 47.- Friese, 1969. Bericht 10. Wandervers. Deutscher Ent., Tagungsb. 80(2): 219.- Brock, 1971. Jour. nat. Hist. 5: 34, 44, 48.- Razowski, 1974. Acta zool. cracov. 19: 6, 9, 11, 14, 16.- Common, 1975. Ann. Rev. Ent. 20: 183.- Speidel, 1977. Atalanta 8: 120.- Gómez B., 1978. Shilap, Revta lepid. 6: 246, 249, fig., 250, 251; syn.: Jugatae, Isoneura.- Gómez B., 1979. Shilap, Revta lepid. 7: 169; syn.: Jugatae, Isoneura.Hennig, 1986. Taschenb. Zool. 3, Wirbellose II. $4^{\text {a }}$ ed., p. 231; syn.: Jugata.- Scoble, 1989, in Emmet \& Head. Moths and Butt. Great Brit. and Ireland 7, p. 17, 18.- Scoble, 1992. Lep., p. 190, 191.- K. Brown, 1996, in Bicudo \& Menezes. Biodiv. Brasil, p. 224.

Heteroneura Tillyard, 1918. Proc. Linn. Soc. N. S. Wales 43: 297, 304, 315; subordem oposta à Homoneura, incluindo as demais famílias.- Imms, 1930. Gen. Textb. Ent. $2^{\mathrm{a}}$ ed., p. 425.- Kiriakoff, 1946. Lambillionea 46: 24, 62.- Agenjo, 1946. Graellsia 4(3): [7].- Warren, 1947. Entomol. 80: 217.Turner, 1947. Proc. Linn. Soc. N. S. Wales 71: 307, 309, 337; syn.: Frenata.- Kiriakoff, 1948. Biol. Jaarboek, Antwerpen, 15, p. 128.- Bourgogne, 1951, in Grassé. Traité Zool. 10(1), p. 373; syn.: Frenatae, Anisoneuria.- Hennig, 1953. Beitr. Ent. 3, Sonderh., p. 47.- Friese, 1969. Bericht 10. Wandervers. Deutscher Ent., Tagungsb. 80(2): 218, 219, 220; syn.: Frenatae, Anisoneuria.- Brock, 1971. Jour. nat.
Hist. 5: 34, 48.- Razowski, 1974. Acta zool. cracov. 19: 6, 9, 11, 14-16.- Common, 1975. Ann. Rev. Rev. 20: 183.Speidel, 1977. Atalanta 8: 120, 121.- Gómez B., 1978. Shilap, Revta lepid. 6: 246, 249, fig., 250; syn.: Frenatae, Anisoneura.- Gómez B., 1979. Shilap, Revta lepid. 7: 170; syn.: Frenatae, Anisoneura.- Kristensen \& Nielsen, 1981, in Cederholm. Ent. scand., Suppl. 15: 301, 303; syn.: Frenatae.- Kristensen \& Nielsen, 1983. Ztschr. zool. Syst. Evolut.-forsch. 21: 116.- Kristensen, 1984. Steenstrupia 10: 162, 163, 165, 186.- Heppner, 1984. Atlas neotrop. Lep. 2. Checkl. 1, p. XX.- Minet, 1984. Nouv. Rev. Ent. (n. s.) 1: 143, 148.- Kristensen, 1985. Ent. Medd. 52: 9.- Nielsen, 1985. Jour. Res. Lep., Suppl. 1: 3, 15; syn.: Frenata.- Davis, 1986. Smiths. Contr. Zool. 434: 55.- Minet, 1986. Alexanor 14: 291, 292.- Hennig, 1986. Taschenb. Zool. 3, Wirbellose

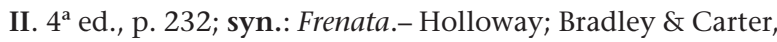
1987. CIE Guides ins. import. man. 1. Lepidoptera, p. 18, 23, 28, 29, 37.- Nielsen, 1989, in Fernholm; Bremer \& Jörnvall (Ed.). Hierarchy Life, p. 284, 285, 286; syn.: Frenata.- Scoble, 1989, in Emmet \& Head. Moths and Butt. Great Brit. and Ireland 7, p. 15-24; syn.: Frenatae.- Nielsen \& Common, 1991, in CSIRO. Ins. Australia, p. 832, 833, 837, 841; syn.: Frenata.- Scoble, 1992. Lep., p. 188, 190, 191, 203, 213.- Heppner, 1995. Atlas neotrop. Lep. 3. Checkl. 2, p. XIII.- K. Brown, 1996, in Bicudo \& Menezes. Biodiv. Brasil, p. 224.- Poole, 1996, in Poole \& Gentili. Nom. Ins. Nearct. 3, p. 613.- Heppner, 1996. Atlas neotrop. Lep. 5B. Checkl. 4B, p. XV.- Nielsen \& Kristensen, 1996. Invert. Tax. 10: 1200, 1253, 1256, 1259, 1262, 1263, 1267-1272, 1274-1276, 1278-1280, 1282, 1283, 1285-1288, 1291, 1292, 1294.- Heppner, 1998. Lep. News 1998 (3): 57.- Heppner, 1998. Holarct. Lep. 5, Suppl. 1: 7, 18.- Kristensen \& Skalski, 1999, in Kristensen (Ed.), Lep. 1, p. 10, 11, 12, 18, in Fischer (Ed.). Handb. Zool. 4.- Carter \& Kristensen, 1999, in Kristensen (Ed.), Lep. 1, p. 28, in Fischer (Ed.). Handb. Zool. 4.- Davis, 1999, in Kristensen (Ed.), Lep. 1, p. 65, in Fischer (Ed.). Handb. Zool. 4.- Powell et al., 1999, in Kristensen (Ed.), Lep. 1, p. 407, in Fischer (Ed.). Handb. Zool. 4.Heppner, 2003. Lep. Florida 1, p. 71, 91, 94, 231; infraordem.

Amplexiformia Tillyard, 1918. Proc. Linn. Soc. N. S. Wales 43: 297; sem status, incluindo os não Jugata e Frenata.

Coronofrenatae Krausse \& Wolff, 1919. Arch. Natg. 86A(3): 165; seção, incluindo Tineaemorpha, Tortricimorpha, Pyralimorpha.- Wolff \& Krausse, 1930. Wirbell. Tiere, p. 199, 200.- Kiriakoff, 1946. Lambillionea 46: 11.

Tineaemorpha Krausse \& Wolff, 1919. Arch. Natg. 86A(3): 165; tribo, incluindo Tineidae, Nepticulidae, Heliozelidae, Tischeriidae, Incurvariidae, Monopidae, Acrolepiidae, Hyponomeutidae, Scythrididae (= Butalididae), Tinegeriidae, Elachistidae, Cemiostomidae, Lyonetiidae, Phyllocnistidae, Oenophilidae, Gracilariidae, Momphidae (= Lavernidae), Heliotinidae, Gelechiidae, Ochsenheimeriidae, Atychiidae, 
Glyphipterygidae, Sesiidae, Psychididae.- Wolff \& Krausse, 1930. Wirbell. Tiere, p. 200.

Tortricimorpha Krausse \& Wolff, 1919. Arch. Natg. 86A(3): 165; tribo, incluindo Tortricidae, Cossidae.- Wolff \& Krausse, 1930. Wirbell. Tiere, p. 200, 201.

Pyralimorpha Krausse \& Wolff, 1919. Arch. Natg. 86A(3): 165; tribo, incluindo Pyralididae, Thyrididae, Pterophoridae, Orneodidae (= Alucitidae).- Wolff \& Krausse, 1930. Wirbell. Tiere, p. 200.

Nudifrenatae Krausse \& Wolff, 1919. Arch. Natg. 86A(3): 165; secão, incluindo Cochlidimorpha.- Wolff \& Krausse, 1930. Wirbell. Tiere, p. 199, 201.- Kiriakoff, 1946. Lambillionea 46: 114.

Cochlidimorpha Krausse \& Wolff, 1919. Arch. Natg. 86A(3): 165; tribo, incluindo Cochlididae.- Wolff \& Krausse, 1930. Wirbell. Tiere, p. 201.

Semicoronofrenatae Krausse \& Wolff, 1919. Arch. Natg. 86A(3): 165, 166; seção, incluindo Zygaenaemorpha, Arctiaemorpha, Geometraemorpha, Noctuaemorpha, Bombycimorpha, Sphingimorpha, Grypoceromorpha, Rhopaloceromorpha.- Wolff \& Krausse, 1930. Wirbell. Tiere, p. 199, 201.- Kiriakoff, 1946. Lambillionea 46: 114.

Zygaenaemorpha Krausse \& Wolff, 1919. Arch. Natg. 86A(3): 166; tribo, incluindo Zygaenidae (= Anrthroceridae), Heterogynidae.Wolff \& Krausse, 1930. Wirbell. Tiere, p. 201.

Arctiaemorpha Krausse \& Wolff, 1919. Arch. Natg. 86A(3): 166; tribo, incluindo Arctiidae, Lithosiidae, Syntomidae, Nycteolidae (= Cymbidae), Nolidae.- Wolff \& Krausse, 1930. Wirbell. Tiere, p. 201, 202.

Geometraemorpha Krausse \& Wolff, 1919. Arch. Natg. 86A(3): 166; tribo, incluindo Geometridae, Uraniidae.- Wolff \& Krausse, 1930. Wirbell. Tiere, p. 201.

Noctuaemorpha Krausse \& Wolff, 1919. Arch. Natg. 86A(3): 166; tribo, incluindo Noctuidae, Agaristidae, Cymatophoridae.Wolff \& Krausse, 1930. Wirbell. Tiere, p. 201.

Bombycimorpha Krausse \& Wolff, 1919. Arch. Natg. 86A(3): 166; tribo, incluindo Bombycidae, Epicopiidae, Lymantriidae (= Liparidae), Thaumetopoeidae, Lasiocampidae, Lemoniidae, Striphnopterygidae (= Eupteroptidae), Endromididae, Drepanidae, Callidulidae, Saturniidae, Brahmaeidae, Notodontidae.- Wolff \& Krausse, 1930. Wirbell. Tiere, p. 201.

Sphingimorpha Krausse \& Wolff, 1919. Arch. Natg. 86A(3): 166; tribo, incluindo Sphingidae.- Wolff \& Krausse, 1930. Wirbell. Tiere, p. 201.

Rhopaloceromorpha Krausse \& Wollf, 1919. Arch. Natg. 86A(3): 166; tribo, incluindo Heliconidae, Acraeidae, Nymphalidae, Danaidae, Brassolidae, Morphididae, Satyridae, Erycinidae, Lycaenidae, Pierididae, Papilionidae.- Wolff \& Krausse, 1930. Wirbell. Tiere, p. 201.

Micróptera [sic] Börner, 1920, in Brohmer. Fauna Deutschland. $2^{\text {a }}$ ed., p. 342; syn.: Microfrenatae, Stemmatoncopoda; grupo superior, incluindo os grupos Acanthopleona e Lipacanthina.
Acanthopleóna [sic] Börner, 1920, in Brohmer. Fauna Deutschland. $2^{a}$ ed., p. 345; grupo, incluindo Gracilarioidea, Tineoidea.- Handlirsch, 1925, in Schröder. Handb. Ent. 3, p. 877.- Börner, 1939. Verh. VII intern. Kongr. Ent., Berlin, 2, p. 1373.- Kiriakoff, 1946. Lambillionea 46: 112.Razowski, 1974. Acta zool. cracov. 19: 9.

Lipacánthina [sic] Börner, 1920, in Brohmer. Fauna Deutschland. $2^{\text {a }}$ ed., p. 345; grupo, incluindo Gelechioidea, Pyralidoidea.- Handlirsch, 1925, in Schröder. Handb. Ent. 3, p. 882.- Börner, 1939. Verh. VII intern. Kongr. Ent., Berlin, 2, p. 1373.- Kiriakoff, 1946. Lambillionea 46: 112.Razowski, 1974. Acta zool. cracov. 19: 9.

Macróptera [sic] Börner, 1920, in Brohmer. Fauna Deutschland. $2^{a}$ ed., p. 349 ; syn.: Harmoncopoda, Macrofrenatae, Macroheterocera + Rhopalocera; grupo superior, incluindo Arctioidea, Noctuoidea, Bombycoidea, Rhopalocera.

Frenati [sic]; Lindsey, 1925. Ann. Ent. Soc. Amer. 18: 87.

Monotrysia Börner, 1925, in Brohmer. Fauna Deutschland. $3^{\text {a }}$ ed., p. 358, 370; subordem, incluindo Hepiólidae [sic], Micropterygidae, Chapmaniidae, Eriocranidae, Nepticulidae, Tischeriidae, Incurvariidae, Adelidae, Heliozelidae.- Börner, 1932, in Brohmer. Fauna Deutschland. $4^{\mathrm{a}}$ ed., p. 369, 381.- Börner, 1939. Verh. VII intern. Kongr. Ent., Berlin, 2 (1938), p. 1373, 1375, 1386, 1401, 1403, 1422.- Le Marchand, 1945. Rev. franç. Lép. 10: 138, 140; syn.: Monostomiogyna.- Hinton, 1946. Trans. ent. Soc. London 97: 4.- Kiriakoff, 1946. Lambillionea 46: 24.- Agenjo, 1946. Graellsia 4(3): [7, 8].Kiriakoff, 1948. Biol. Jaarboek, Antwerpen, 15, p. 129, 130, 131, 132, 136.- Börner, 1949, in Brohmer. Fauna Deutschland. $6^{a}$ ed., p. 382, 395.- Bourgogne, 1951, in Grassé. Traité Zool. 10(1), p. 373; syn.: Tineides Aculeatae, Adéliformes, Stigmellina.- Hennig, 1953. Beitr. Ent. 3, Sonderh., p. 47.Richards \& Davies, 1957, in Imms. Gen. Textb. Ent., p. 533, 534, 538.- Friese, 1969. Bericht 10. Wandervers. Deutscher Ent., Tagungsb. 80(2): 207, 208, 213.- Common, 1970, in CSIRO. Ins. Australia, p. 782, 783, 784, 787.- Brock, 1971. Jour. nat. Hist. 5: 34.- Razowski, 1974. Acta zool. cracov. 19: 9, 11.- Common, 1974, in CSIRO. Ins. Australia, p. 103.Dugdale, 1974. N. Zealand Jour. Zool. 1: 129, 136, 143.Birkit-Smith, 1974. Ent. scand. 5: 161, 173.- Kuitjen, 1974. Neth. Jour. Zool. 24: 320.- Common, 1975. Ann. Rev. Ent. 20: 183, 196.- Speidel, 1977. Atalanta 8: 121.- Gómez B., 1978. Shilap, Revta lepid. 6: 246, 247, 247bis, fig., 251, 257.Gómez B., 1979. Shilap, Revta lepid. 7: 169.- Küppers \& Speidel, 1980. Atalanta 11: 58.- Heppner, 1984. Atlas neotrop. Lep. 2. Checkl. 1, p. XX.- Davis, 1986. Smiths. Contr. Zool. 434: 1.- Holloway; Bradley \& Carter, 1987. CIE Guides ins. import. man. 1. Lepidoptera, p. 18, 23, 28, 37.Nielsen, 1989, in Fernholm; Bremer \& Jörnvall (Ed.). Hierarchy Life, p. 281.- Scoble, 1989, in Emmet \& Head. Moths and Butt. Great Brit. and Ireland 7, p. 18.- Niculescu, 1989. Dtsch. ent. Ztschr. (N. F.) 36: 335.- Scoble, 1992. Lep., p. 190, 191.- Heppner, 1995. Atlas neotrop. Lep. 3. Checkl.

Revista Brasileira de Zoologia 23 (1): 1-41, março 2006 
2, p. XIII.- Heppner, 1996. Atlas neotrop. Lep. 5B. Checkl. 4B, p. XV.- Heppner, 1998. Lep. News 1998 (3): 57.- Heppner, 1998. Holarct. Lep. 5, Suppl. 1: 7, 18.- Covell, 1999. Kentucky State Nat. Pres. Comm., Sc. Tech. Ser. 6: XI, 15; subordem.- Davis, 1999, in Kristensen (Ed.), Lep. 1, p. 65, in Fischer (Ed.). Handb. Zool. 4.- Heppner, 2003. Lep. Florida 1, p. 71, 91, 94, 231; divisão.

Ditrysia Börner, 1925, in Brohmer. Fauna Deutschland. $3^{\text {a }}$ ed., p. 371; subordem oposta à Monotrysia, incluindo as demais famílias.- Börner, 1932, in Brohmer. Fauna Deutschland. $4^{\mathrm{a}}$ ed., p. 369, 383.- Börner, 1939. Verh. VII intern. Kongr. Ent., Berlin, 2(1938), p. 1373, 1375, 1386, 1401, 1407, 1422; subordem, incluindo Heterocera, Rhopalocera.- Le Marchand, 1945. Rev. franç. Lép. 10: 138; syn.: Distomiogyna.- Hinton, 1946. Trans. ent. Soc. London 97: 4.- Kiriakoff, 1946. Lambillionea 46: 24.- Agenjo, 1946. Graellsia 4(3): [7, 8].Kiriakoff, 1948. Biol. Jaarboek, Antwerpen, 15, p. 129, 130, 131, 136, 140, 141.- Börner, 1949, in Brohmer. Fauna

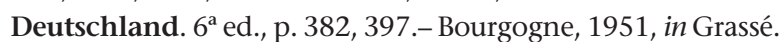
Traité Zool. 10(1), p. 376; syn.: Distomiogyna.- Hennig, 1953. Beitr. Ent. 3, Sonderh., p. 47.- Kiriakoff, 1955. Bull. \& Ann. Soc. ent. Belg. 91: 155-157.- Richards \& Davies, 1957, in Imms. Gen. Textb. Ent., p. 533, 534, 539.- Friese, 1969. Bericht 10. Wandervers. Deutscher Ent., Tagungsb. 80(2): 207, 211, 213, 214, 218, 219, 220; syn.: Distomiogyna.Common, 1970, in CSIRO. Ins. Australia, p. 782, 783, 784, 793.- Brock, 1971. Jour. nat. Hist. 5: 34-99.- Common, 1974, in CSIRO. Ins. Australia, p. 104.- Dugdale, 1974. N. Zealand Jour. Zool. 1: 129, 140, 143.- Razowski, 1974. Acta zool. cracov. 19: 9, 10, 13, 16.- Birkit-Smith, 1974. Ent. scand. 5: 161, 173.- Kuitjen, 1974. Neth. Jour. Zool. 24: 320.Common, 1975. Ann. Rev. Ent. 20: 183, 197, 200.- Speidel, 1977. Atalanta 8: 121.- Gómez B., 1978. Shilap, Revta lepid. 7: 246, 247, 247bis, fig., 248, 251, 257.-Kuznetzov \& Stekolnikov, 1978. Ent. Obozr. 57: 871, 872.- Gómez B., 1979. Shilap, Revta lepid. 7: 170.- Kuznetsov \& Stekolnikov, 1979. Ent. Rev. 57: 613.- Kristensen \& Nielsen, 1981, in Cederholm. Ent. scand., Suppl. 15: 301, 302, 303.- Minet, 1983. Ann. Soc. ent. France (n. s.) 19(2): 179, 180.- Heppner, 1984. Atlas neotrop. Lep. 2. Checkl. 1, p. XX.- Minet, 1984. Nouv. Rev. Ent. (n. s.) 1: 145-148.- Kristensen, 1985. Ent. Medd. 52: 9.Nielsen, 1985. Jour. Res. Lep., Suppl. 1: 16.- Minet, 1986. Alexanor 14: 292.- Hennig, 1986. Taschenb. Zool. 3, Wirbellose II. $4^{\text {a }}$ ed., p. 232.- Minet, 1986. Alexanor 14: 292.- Holloway; Bradley \& Carter, 1987. CIE Guides ins. import. man. 1. Lepidoptera, p. 19, 23, 29, 37.- Nielsen, 1989, in Fernholm; Bremer \& Jörnvall (Ed.). Hierarchy Life, p. $281,282,284,285,287,288,291$.- Scoble, 1989, in Emmet \& Head. Moths and Butt. Great Brit. and Ireland 7, p. 1618, 24, 26.- Niculescu, 1989. Dtsch. ent. Ztschr. (N. F.) 36: 335.- Nielsen \& Common, 1991, in CSIRO. Ins. Australia, p. 832, 833, 845.- Minet, 1991. Ent. scand. 22: 69, 70, 80, 83.Scoble, 1992. Lep., p. 188, 190, 191, 205, 225, 290.- Heppner,
1995. Atlas neotrop. Lep. 3. Checkl. 2, p. XIII.- de Jong; Vane-Wright \& Ackery, 1996. Ent. scand. 27: 72.- Poole, 1996, in Poole \& Gentili. Nom. Ins. Nearct. 3, p. 613.- Heppner, 1996. Atlas neotrop. Lep. 5B. Checkl. 4B, p. XV.- Nielsen \& Kristensen, 1996. Invert. Tax. 10: 1256, 1258, 1282, $1294 .-$ Heppner, 1998. Lep. News 1998 (3): 58.- Heppner, 1998. Holarct. Lep. 5, Suppl. 1: 7, 18.- Covell, 1999. Kentucky State Nat. Pres. Comm., Sc. Tech. Ser. 6: XI, 18; subordem.Kristensen \& Skalski, 1999, in Kristensen (Ed.). Lep. 1, p. 10, 12, 14, in Fischer (Ed.). Handb. Zool. 4.- Carter \& Kristensen, 1999, in Kristensen (Ed.), Lep. 1, p. 28, 29, in Fischer (Ed.). Handb. Zool. 4.- Powell et al., 1999, in Kristensen (Ed.), Lep. 1, p. 56, in Fischer (Ed.). Handb. Zool. 4.- Heppner, 2003. Lep. Florida 1, p. 71, 91, 94, 231; divisão.

Nepticulina Meyrick, 1928. Rev. Handb. brit. Lep., p. 29, 846; filo, incluindo Nepticulidae.- Börner, 1939. Verh. VII intern. Kongr. Ent., Berlin, 2, p. 1407, 1423.- Le Marchand, 1945. Rev. franç. Lép. 10: 110.- Turner, 1947. Proc. Linn. Soc. N. S. Wales 71: 305.- Kiriakoff, 1948. Biol. Jaarboek, Antwerpen, 15, p. 135.- Kristensen, 1984. Steenstrupia 10: 141.- Minet, 1984. Nouv. Rev. Ent. (n. s.) 1: 141, 143, 145, 147, 148; syn.: Nannolepidoptera.- Kristensen, 1985. Ent. Medd. 52: 9.- Minet, 1986. Alexanor 14: 291, 292.Heppner, 1995. Atlas neotrop. Lep. 3. Checkl. 2, p. XIII.Heppner, 1996. Atlas neotrop. Lep. 5b. Checkl. 4B, p. XV.Heppner, 1998. Lep. News 1998 (3): 57.- Heppner, 1998. Holarct. Lep. 5, Suppl. 1: 7, 18.- Davis, 1999, in Kristensen (Ed.), Lep. 1, p. 65, in Fischer (Ed.). Handb. Zool. 4.Heppner, 2003. Lep. Florida 1, p. 71, 91, 94, 231; seção.

Drepanina Meyrick, 1928. Rev. Handb. brit. Lep., p. 29, 371; filo, incluindo Drepanidae, Callidulidae.- Le Marchand, 1945. Rev. franç. Lép. 10: 110.- Turner, 1947. Proc. Linn. Soc. N. S. Wales 71: 305 .

Holopoda H. von Ihering, 1929. Ent. Rdsch., Stuttgart, 46; sep., p. 6; divisão de Rhopalocera, incluindo Papilionidae, Pieridae, Lycaenidae, Erycinidae, Danaidae, Acraeidae.

Peropoda H. von Ihering, 1929. Ent. Rdsch., Stuttgart, 46; sep., p. 6-7; divisão de Rhopalocera, incluindo Nymphalidae, Satyridae.

Jugates [sic]; Lameere, 1931. Rec. Inst. zool. Torley-Rousseau 3(2): 235.- Lameere, 1936. Bull. \& Ann. Soc. ent. Belg. 76: 407, 413; syn.: Homoneures.- Lameere, 1938. Rec. Inst. zool. Torley-Rouseau 5: 168, 169, 177, 194, 195; syn.: Homoneures.

Frénates [sic]; Lameere, 1931. Rec. Inst. zool. Torley-Rousseau 3(2): 235.- Lameere, 1936. Bull. \& Ann. Soc. ent. Belg. 76: 407, 413; syn.: Hétéroneures.- Lameere, 1938. Rec. Inst. zool. Torley-Rousseau 5: 168, 169, 177, 194, 200; syn.: Hétéroneures. Coronates Lameere, 1931. Rec. Inst. zool. Torley-Rousseau 3(2): 235; sem status, oposto a Sémicoronates, incluindo Cossus, Castnia, Psyche, Zygaena, Tinea, Sesia, Pterophorus, Tortrix, Pyralis, Hydrocampa, Galleria.

Revista Brasileira de Zoologia 23 (1): 1-41, março 2006 
Sémicoronates Lameere, 1931. Rec. Inst. zool. Torley-Rousseau 3(2): 235; sem status, oposto a Coronates, incluindo Hétérocères e Rhopalocères.

Adeliformes Lameere, 1936. Bull. \& Ann. Soc. ent. Belg. 76: 408, 413; syn.: Tinéoides aculéates; grupo, incluindo Adélides, Nepticulides.- Lameere, 1938. Rec. Inst. zool. Torley-Rousseau 5: 194, 202.- Friese, 1969. Bericht 10. Wandervers. Deutscher Ent., Tagungsb. 80(2): 218; syn.: Tineides aculeatae, Stigmellina.- Razowski, 1974. Acta zool. cracov. 19: 10.

Tineiformes Lameere, 1936. Bull. \& Ann. Soc. ent. Belg. 76: 409, 413; grupo, incluindo Tineoïdes, Gelechioïdes.- Lameere, 1938. Rec. Inst. zool. Torley-Rousseau 5: 194.- Kiriakoff, 1948. Biol. Jaarboek, Antwerpen, 15, p. 142; subcohors.Heppner, 1996. Atlas neotrop. Lep. 5B. Checkl. 4B, p. XV; série, infra-superfamília.- Heppner, 1998. Lep. News 1998 (3): 58.- Heppner, 1998. Holarct. Lep. 5, Suppl. 1: 18.

Pyraliformes Lameere, 1936. Bull. \& Ann. Soc. ent. Belg. 76: 410, 413; grupo, incluindo Hyponomeutoïdes, Pyraloïdes.Lameere, 1938. Rec. Inst. zool. Torley-Rousseau 5: 194, 220.

Papilioniformes Lameere, 1936. Bull. \& Ann. Soc. ent. Belg. 76: 410, 413; grupo, incluindo Rhopalocères.- Lameere, 1938. Rec. Inst. zool. Torley-Rousseau 5: 169, 194, 229.- Kiriakoff, 1948. Biol. Jaarboek, Antwerpen, 15, p. 142.- Kiriakoff, 1952. Ann. Soc. r. zool. Belg. 83: 99.- Heppner, 1995. Atlas neotrop. Lep. 3. Checkl. 2, p. XIV; infra-superfamília.Heppner, 1996. Atlas neotrop. Lep. 5B. Checkl. 4B, p. XVI; infra-superfamília.- Heppner, 1998. Lep. News 1998 (3): 59.Heppner, 1998. Holarct. Lep. 5, Suppl. 1: 21.

Cossiformes Lameere, 1936. Bull. \& An. Soc. ent. Belg. 76: 411, 413; grupo, incluindo Cossoïdes, Castniö̈des.- Lameere, 1938. Rec. Inst. zool. Torley-Rousseau 5: 194.- Heppner, 1998. Lep. News 1998 (3): 58; série, infra-superfamília.- Heppner, 1998. Holarct. Lep. 5, Suppl. 1: 20.

Geometriformes Lameere, 1936. Bull. \& Ann. Soc. ent. Belg. 76: 412, 413; grupo, incluindo Cymatophoroïdes, Géometroïdes.Lameere, 1938. Rec. Inst. zool. Torley-Rousseau 5: 175, 194.Heppner, 1984. Atlas neotrop. Lep. 2. Checkl. 1, p. XXII; série de Geometroidea, infra-superfamília.

Noctuiformes Lameere, 1936. Bull. \& Ann. Soc. ent. Belg. 76: 412, 413; grupo, incluindo Noctuoïdes, Notodontoïdes.Lameere, 1938. Rec. Inst. zool. Torley-Rousseau 5: 175, 179, 194.- Kiriakoff, 1948. Biol. Jaarboek, Antwerpen, 15, p. 142; subcohors.- Kiriakoff, 1952. Ann. Soc. r. zool. Belg. 83: 99.

Zygéniformes Lameere, 1936. Bull. \& Ann. Soc. ent. Belg. 76: 409, 413; grupo, incluindo Zygénides, Hétérogynides, Megalopygides, Cochlidiides.- Lameere, 1938. Rec. Inst. zool. TorleyRousseau 5: 194, 217.

Homoneures [sic]; Lameere, 1938. Rec. Inst. zool. TorleyRousseau 5: 195.

Heteroneures [sic]; Lameere, 1938. Rec. Inst. zool. TorleyRousseau 5: 200.

Microlépidoptères [sic]; Lameere, 1938. Rec. Inst. zool. Torley-
Rousseau 5: 194.

Macrolépidoptères [sic]; Lameere, 1938. Rec. Inst. zool. TorleyRousseau 5: 194.

Eulepidoptera Börner, 1939. Verh. VII intern. Kongr. Ent., Berlin, 2, p. 1372 (não define; atribuido a Spuler, 1910 (não localizado]).- Kiriakoff, 1948. Biol. Jaarboek, Antwerpen, 15, p. 137, 138; supercohors, incluindo Stemmatoncopoda e Harmoncopoda.- Hennig, 1953. Beitr. Ent. 3, Sonderh., p. 47.- Kiriakoff, 1955. Bull. \& Ann. Soc. ent. Belg. 91: 155158.- Friese, 1969. Bericht 10. Wandervers. Deutscher Ent., Tagungsb. 80(2): 208.- Razowski, 1974. Acta zool. cracov. 19: 10, 15.- Gómez B., 1978. Shilap, Revta lepid. 6: 246.Minet, 1984. Nouv. Rev. Ent. (n. s.) 1: 141, 143, 145, 147, 148.- Minet, 1986. Alexanor 14: 291.- Nielsen, 1989, in Fernholm; Bremer \& Jörnvall (Ed.). Hierarchy Life, p. 282. Incurvariina Börner, 1939. Verh. VII intern. Kongr. Ent. 2, p. 1407, 1423; infrasérie de Micropterygoidea, incluindo Eriocraniidae, Incurvariidae, Adelidae, Heliozelidae, Prodoxidae.Hennig, 1953. Beitr. Ent. 3, Sonderh., p. 47.- Kiriakoff, 1955. Bull. \& Ann. Soc. ent. Belg. 91: 155-157.- Razowski, 1974. Acta zool. cracov. 19: 10.- Minet, 1984. Nouv. Rev. Ent. (n. s.) 1: 145, 147, 148.- Kristensen, 1985. Ent. Medd. 52: 9.- Minet, 1986. Alexanor 14: 292.- Nielsen, 1989, in Fernholm; Bremer \& Jörnvall (Ed.). Hierarchy Life, p. 282.Heppner, 1996. Atlas neotrop. Lep. 5B. Checkl. 4B, p. XV.Nielsen \& Kristensen, 1996. Invert. Tax. 10: 1262.- Heppner, 1998. Lep. News 1998 (3): 57.- Heppner, 1998. Holarct. Lep. 5, Suppl. 1: 7, 18.- Davis, 1999, in Kristensen (Ed.), Lep. 1, p. 65, in Fischer (Ed.). Handb. Zool. 4.- Heppner, 2003. Lep. Florida 1, p. 71, 91, 94, 231; seção.

Platyptères Rambur, 1942. Faune ent. de L'Andalousie. $2^{\mathrm{a}}$ ed., p. 239; tribo, incluindo Papilionides, Pierides, Polyommatides, Nymphalides, Libythéides, Satyrides.

Microptères [sic]; Rambur, 1942. Fauna ent. de L'Andalousie. $2^{\mathrm{a}}$ ed., p. 303; tribo, incluindo Hespérides.- Kiriakoff, 1946. Lambillionea 46: 112.

Stigmellina Le Marchand, 1945. Rev. franç. Lép. 10: 130, 138; filo, incluindo Incurvarioidea, Stigmelloidea.

Monostomiogyna Le Marchand, 1945. Rev. franç. Lép. 10: 105, 130; grupo, incluindo Incurvariidae, Adelidae, Heliozelidae, Crinopterygidae, Tischeriidae, Nepticulidae, Opostegidae.Kiriakoff, 1946. Lambillionea 46: 24.- Razowski, 1974. Acta zool. cracov. 19: 9.- Agenjo, 1946. Graellsia 4(3): [7].

Distomiogyna Le Marchand, 1945. Rev. franç. Lép. 10: 105; grupo oposto à Monostomiogyna, incluindo as demais famílias.Kiriakoff, 1946. Lambillionea 46: 24.- Agenjo, 1946. Graellsia 4(3): [7].- Razowski, 1974. Acta zool. cracov. 19: 9.

Heterotrysia Agenjo, 1946. Graellsia 4(3): [7], tab.; falange, incluindo Psychoidea.

Tineaea aculeatae [sic]; Kiriakoff, 1946. Lambillionea 46: 57. Macroptères [sic]; Kiriakoff, 1946. Lambillionea 46: 112.

Dacnonypha Hinton, 1946. Trans. ent. Soc. London 97: 3, 4;

Revista Brasileira de Zoologia 23 (1): 1-41, março 2006 
subordem, incluindo Mnesarchaeidae e Eriocraniidae.Kiriakoff, 1948. Biol. Jaarboek, Antwerpen, 15, p. 130.Hennig, 1953. Beitr. Ent. 3, Sonderh., p. 47.- Kiriakoff, 1955. Bull. \& Ann. Soc. ent. Belg. 91: 155, 156, 158; syn.: Hoplostomatoptera.- Friese, 1969. Bericht 10. Wandervers. Deutscher Ent., Tagungsb. 80(2): 218; syn.: Paleolepidoptera, Hoplostomatoptera.- Brock, 1971. Jour. nat. Hist. 5: 34, 48.Common, 1974, in CSIRO. Ins. Australia, Suppl., p. 101.Dugdale, 1974. N. Zealand Jour. Zool. 1: 129, 134, 143.Razowski, 1974. Acta zool. cracov. 19: 9-11.- Common, 1975. Ann. Rev. Ent. 20: 195, 200.- Speidel, 1977. Atalanta 8: 119, 120, 121.- Gómez B., 1978. Shilap, Revta lepid. 6: 247, 247bis, fig., 251, 257.- Gómez B., 1979. Shilap, Revta lepid. 7: 169.- Küppers \& Speidel, 1980. Atalanta 11: 5759.- Kristensen \& Nielsen, 1981, in Cederholm. Ent. scand., Suppl. 15: 300, 301, 303, 304.- Kristensen \& Nielsen, 1983. Ztschr. zool. Syst. Evolut.-forsch. 21: 115, 116.- Kristensen, 1984. Steestrupia 10: 161, 170, 186.- Heppner, 1984. Atlas neotrop. Lep. 2. Checkl. 1, p. XX.- Minet, 1984. Nouv. Rev. Ent. (n. s.) 1: 147, 148.- Nielsen, 1985. Jour. Res. Lep., Suppl. 1: 15.- Kristensen, 1985. Ent. Medd. 52: 9.- Minet, 1986. Alexanor 14: 291, 292.- Holloway; Bradley \& Carter, 1987. CIE Guides ins. import. man. 1. Lepidoptera, p. 18, 23, 25, 38.- Nielsen, 1989, in Fernholm; Bremer \& Jörnvall (Ed.). Hierarchy Life, p. 282, 285.- Scoble, 1989, in Emmet \& Head. Moths and Butt. Great Brit. and Ireland 7, p. 15, 17, 21.- Niculescu, 1989. Dtsch. ent. Ztschr. (N. F.) 36: 335.Nielsen \& Common, 1991, in CSIRO. Ins. Australia, p. 832.Scoble, 1992. Lep., p. 188, 195, 203, 205.- Heppner, 1995. Atlas neotrop. Lep. 3. Checkl. 2, p. XIII.- Poole, 1996, in Poole \& Gentili. Nom. Ins. Nearct. 3, p. 612.- Heppner, 1996. Atlas neotrop. Lep. 5B. Checkl. 4B, p. XV.- Nielsen \& Kristensen, 1996. Invert. Tax. 10: 1200, 1201, 1262, 1292, 1293.- Heppner, 1998. Lep. News 1998 (3): 57; cohorte e infraordem.- Heppner, 1998. Holarct. Lep. 5, Suppl. 1: 7, 18; cohorte e infraordem.- Covell, 1999. Kentucky State Nat. Pres. Comm., Sc. Tech. Ser. 6: XI, 15; subordem.Kristensen, 1999, in Kristensen (Ed.), Lep. 1, p. 51, in Fischer (Ed.). Handb. Zool. 4.- Heppner, 2003. Lep. Florida 1, p. 71, 91, 94, 231; coorte e infraordem.

Asthenochorda Turner, 1947. Proc. Linn. Soc. N. S. Wales 71: 310; divisão, incluindo Rhopalocera, Microptila.- Razowski, 1974. Acta zool. cracov. 19: 9, 10.

Microptila Turner, 1947. Proc. Linn. Soc. N. S. Wales 71: 311, 318, 337; subdivisão, incluindo Zygaenoidea, Pyraloidea (Pyralidoidea), Pterophoroidea, Tineaoidea.- Razowski, 1974. Acta zool. cracov. 19: 9, 10.

Sthenochorda Turner, 1947. Proc. Linn. Soc. N. S. Wales 71: 310, 311; divisão, incluindo os Heteroneura, não incluidos nos Asthenochorda.

Hoplostomatoptera Kiriakoff, 1948. Biol. Jaarboek, Antwerpen, 15, p. 142; syn.: Eriocraniids; supercohors.- Friese, 1969. Bericht 10. Wandervers. Deutscher Ent., Tagungsb. 80(2):
208.- Razowski, 1974. Acta zool. cracov. 19: 10.- Gómez B., 1978. Shilap, Revta lepid. 6: 246.- Niculescu, 1989. Dtsch. ent. Ztschr. (N. F.) 36: 335.

Aplostomatoptera Kiriakoff, 1948. Biol. Jaarboek, Antwerpen, 15, p. 142; syn.: Hepialids; supercohors.- Hennig, 1953. Beitr. Ent. 3, Sonderh., p. 47.- Kiriakoff, 1955. Bull. \& Ann. Soc. ent. Belg. 91: 155, 156, 158.- Friese, 1969. Bericht 10. Wandervers. Deutscher Ent., Tagungsb. 80(2): 208.Razowski, 1974. Acta zool. cracov. 19: 10.- Küppers \& Speidel, 1980. Atalanta 11: 55, 60-63; syn.: Gymnoptera, Exoporia, Hepialomorpha.- Nielsen, 1989, in Fernholm; Bremer \& Jörnvall (Ed.). Hierarchy Life, p. 282.- Niculescu, 1989. Dtsch. ent. Ztsch. (N. F.) 36: 335.

Nannolepidoptera Kiriakoff, 1948. Biol. Jaarboek, Antwerpen, 15, p. 142; syn.: Nepticulids; supercohors.- Hennig, 1953. Beitr. Ent. 3, Sonderh., p. 47.- Kiriakoff, 1955. Bull. \& Ann. Soc. ent. Belg. 91: 155, 156, 158.- Friese, 1969. Bericht 10. Wandervers. Deutscher Ent., Tagungsb. 80(2): 208.- Nielsen, 1989, in Fernholm; Bremer \& Jörnvall (Ed.). Hierarchy Life, p. 282.- Niculescu, 1989. Dtsch. ent. Ztschr. (N. F.) 36: 335.Gómez B., 1978. Shilap, Revta lepid. 6: 246.

Incurvariiformes Kiriakoff, 1948. Biol. Jaarboek, Antwerpen, 15, p. 142; subcohors, sem indicação de taxa.

Gelechiiformes Kiriakoff, 1948. Biol. Jaarboek, Antwerpen, 15, p. 142; subcohors, sem indicação de taxa.

Pyralidiformes Kiriakoff, 1948. Biol. Jaarboek, Antwerpen, 15, p. 142; subcohors, sem indicação de taxa.

Sphingiformes Kiriakoff, 1948. Biol. Jaarboek, Antwerpen, 15, p. 142; subcohors, sem indicação de taxa.

Zeugloptères [sic]; Kiriakoff, 1952. Ann. Soc. r. zool. Belg. 83: 95, 96.

Hoplostomatoptères [sic]; Kiriakoff, 1952. Ann. Soc. r. zool. Belg. 83: 97.

Aplostomatoptères [sic]; Kiriakoff, 1952. Ann. Soc. r. zool. Belg. 83: 97.

Nannolepidoptères [sic]; Kiriakoff, 1952. Ann. Soc. r. zool. Belg. 83: 97.

Eulépidoptères [sic]; Kiriakoff, 1952. Ann. Soc. r. zool. Belg. 83: 97, 98, 99.

Parasternaria Niculescu, 1970. Bull. Soc. Ent. Mulhouse, jan.févr., p. 15; subordem, incluido Eriocranioidea, Micropterygoidea, Hepialiodea, Incurvarioidea, Cossoidea, Castnioidea, Psychoidea, Tineoidea, Tortricoidea, Zygaenoidea, Pyralidoidea, Geometroidea, Noctuoidea, Bombycoidea, Sphingoidea.- Gómez B., 1979. Shilap, Revta lepid. 7: 168.- Niculescu, 1987. Shilap, Revta lepid. 15: 107-109; subordem, oposta a Aparasternaria, incluindo os Noctuidae e demais mariposas.

Aparasternaria Niculescu, 1970. Bull. Soc. Ent. Mulhouse, jan.févr., p. 14, 15; subordem oposta a Parasternaria, incluindo Hesperioidea e Papilionoidea.- Niculescu, 1987. Shilap, Revta lepid. 15: 107-109.- Gómez B., 1979. Shilap, Revta lepid. 7: 168 .

Revista Brasileira de Zoologia 23 (1): 1-41, março 2006 
Angiocera Friese, 1970. Bericht 10. Wandervers. Deutscher Ent., Tagungsb. 80(2): 218, 220; subordem, oposta a Gymnocera, incluindo todos os grupos, menos Hepialoidea.Kristensen, 1984. Steenstrupia 10: 166.

Gymnocera Friese, 1970. Bericht 10. Wandervers. Deutscher Ent., Tagungsb. 80(2): 218, 220; syn.: Aplostomatoptera; subordem oposta a Angiocera, incluindo Hepialoidea.Küppers \& Speidel, 1980. Atalanta 11: 60.- Kristensen, 1984. Steenstrupia 10: 166.

Dachnonypha [sic]; Common, 1970, in CSIRO. Ins. Australia, p. 782-785.- Birkit-Smith, 1974. Ent. scand. 5: 161, 167, 173.- Gómez B., 1978. Shilap, Revta lepid. 6: 247.

Nennolepidoptera [sic]; Razowski, 1974. Acta zool. cracov. 19: $10,16$.

Exoporian Ditrysia Dugdale, 1974. New Zealand Jour. Zool. 1: 140; grupo, incluindo Mnesarchaeidae, Palaeosetidae, Prototheoridae, Hepialidae.

Endoporian Ditrysia Dugdale, 1974. New Zealand Jour. Zool. 1: 140; grupo oposto à Exoporian Ditrysia, incluindo as demais famílias de Ditrysia.

Exoporia Common, 1975. Ann. Rev. Ent. 20: 196, 200; subordem, incluindo Mnesarchioidea e Hepialoidea.- Speidel, 1977. Atalanta 8: 120, 121.- Gómez B., 1978. Shilap, Revta lepid. 6: 247, fig., 251, 257.- Gómez B., 1979. Shilap, Revta lepid. 7: 169.- Küppers \& Speidel, 1980. Atalanta 11: 60.Kristensen \& Nielsen, 1981, in Cederholm. Ent. scand., Suppl. 15: 301, 303, 304; syn.: Aplostomatoptera.- Kristensen \& Nielsen, 1983. Ztschr. zool. Syst. Evolut.-forsch. 21: 116.- Kristensen, 1984. Steenstrupia 10: 162, 163, 186.Heppner, 1984. Atlas neotrop. Lep. 2. Checkl. 1, p. XX.Minet, 1984. Nouv. Rev. Ent. (n. s.) 1: 147.- Nielsen, 1985. Jour. Res. Lep., Suppl. 1, p. 15.- Kristensen, 1985. Ent. Medd. 52: 9.- Minet, 1986. Alexanor 14: 291, 292.Holloway; Bradley \& Carter, 1987. CIE Guides ins. import. man. 1. Lepidoptera, p. 18, 23, 27, 38.- Nielsen, 1989, in Fernholm; Bremer \& Jörnvall (Ed.). Hierarchy Life, p. 284287, 291.- Scoble, 1989, in Emmet \& Head. Moths and Butt. Great Brit. and Ireland 7, p. 15, 17, 21, 22.- Niculescu, 1989. Dtsch. ent. Ztschr. (N. F.) 36: 335.- Nielsen \& Common, 1991, in CSIRO. Ins. Australia, p. 832, 833, 837.Scoble, 1992. Lep., p. 188, 191, 203, 205.- Heppner, 1995. Atlas neotrop. Lep. 3. Checkl. 2, p. XIII.- Poole, 1996, in Poole \& Gentili. Nom. Ins. Nearct. 3, p. 612.- Heppner, 1996. Atlas neotrop. Lep. 5B. Checkl. 4B, p. XV.- Nielsen \& Kristensen, 1996. Invert. Tax. 10: 1200, 1253, 1256, 1258, 1260-1264, 1267, 1268, 1270-1273, 1275, 1276, 1278- 1280, 1282-1288, 1291, 1292, 1294.- Heppner, 1998. Lep. News 1998 (3): 57.- Heppner, 1998. Holarct. Lep. 5, Suppl. 1: 7, 18.- Covell, 1999. Kentucky State Nat. Pres. Comm., Sc. Tech. Ser. 6: XI, 15; subordem.- Kristensen \& Skalski, 1999, in Kristensen (Ed.), Lep. 1, p. 11, 17, in Fischer (Ed.). Handb. Zool. 4.- Carter \& Kristensen, 1999, in Kristensen (Ed.), Lep. 1, p. 28, in Fischer (Ed.). Handb. Zool. 4.- Kristensen, 1999, in Kristensen (Ed.), Lep. 1, p. 57, in Fischer (Ed.). Handb. Zool. 4.- Powell et al., 1999, in Kristensen (Ed.), Lep. 1, p. 407, in Fischer (Ed.). Handb. Zool. 4.- Heppner, 2003. Lep. Florida 1, p. 71; infraordem.

Aglossata Speidel, 1977. Atalanta 8: 120, 121; [subordem], incluindo Agathiphagidae.- Küppers \& Speidel, 1980. Atalanta 11: 58.- Kristensen \& Nielsen, 1981, in Cederholm. Ent. scand., Suppl. 15: 303.- Kristensen \& Nielsen, 1983. Ztschr. zool. Syst. Evolut.-forsch. 21: 114, 115, 116, 18, 119.Kristensen, 1984. Steenstrupia 10: 157, 170, 186.- Heppner, 1984. Atlas neotrop. Lep. 2. Checkl. 1, p. XIX.- Minet, 1984. Nouv. Rev. Ent. (n. s.) 1: 147.- Nielsen, 1985. Jour. Res. Lep., Suppl. 1: 15.- Minet, 1986. Alexanor 14: 291, 292.- Hennig, 1986. Taschenb. Zool. 3, Wirbellose II. $4^{\text {a }}$ ed., p. 230.- Holloway; Bradley \& Carter, 1987. CIE Guides ins. import. man. 1. Lepidoptera, p. 17, 23, 25, 38.Nielsen, 1989, in Fernholm; Bremer \& Jörnvall (Ed.). Hierarchy Life, p. 281-284, 291.- Scoble, 1989, in Emmet $\&$ Head. Moths and Butt. Great Brit. and Ireland 7, p. 15, 17, 20.- Nielsen \& Common, 1991, in CSIRO. Ins. Australia, p. 831-835.- Scoble, 1992. Lep., p. 188, 192, 195, 197.Heppner, 1995. Atlas neotrop. Lep. 3. Checkl. 2, p. XIII.Heppner, 1996. Atlas neotrop. Lep. 5B. Checkl. 4B, p. XV.Nielsen \& Kristensen, 1996. Invert. Tax. 10: 1200, 1279.Heppner, 1998. Lep. News 1998 (3): 57.- Heppner, 1998. Holarct. Lep. 5, Suppl. 1: 7, 18.- Powell et al., 1999, in Kristensen (Ed.), Lep. 1, p. 405, in Fischer (Ed.). Handb. Zool. 4.- Heppner, 2003. Lep. Florida 1, p. 71; subordem. Aplostommatoptera [sic]; Gómez B., 1978. Shilap, Revta lepid. 6: 246.

Micropterigomorpha Kuznetzov \& Stekolnikov, 1978. Ent. Obozr. 57: 871, 872, 879, 881, 882, 887, 888; infraordem, incluindo Micropterigoidea.- Kuznetzov \& Stekolnikov, 1979. Ent. Rev. 57: 602, 603, 607, 609, 610, 613.- Kristensen \& Nielsen,1983. Ztschr. zool. Syst. Evolut.-forsch. 21: 116.

Eriocraniomorpha Kuznetzov \& Stekolnikov, 1978. Ent. Obozr. 57: 871, 872, 879, 881, 884, 887, 888; syn.: Palaeolepidoptera, Dacnonypha, Haplostomatoptera; infraordem, incluindo Neupseustoidea, Eriocranioidea.- Kuznetzov \& Stekolnikov, 1979. Ent. Rev. 57: 602, 603, 607, 609, 611, 613; syn.: Palaeolepidoptera, Dacnonypha, Haplostomatoptera.

Hepialomorpha Kuznetzov \& Stekolnikov, 1978. Ent. Obozr. 57: 871, 872, 879, 881, 883, 887, 888; syn.: Jugata, Aplostomatoptera, Gymnocera, Exoporian Ditrysia; infraordem, incluindo Hepialoidea.- Kuznetzov \& Stekolnikov, 1979. Ent. Rev. 57: 602, 603, 607, 609, 610, 613; syn.: Jugata, Aplostomatoptera, Gymnocera, Exoporian Ditrysia.

Adelomorpha Kuznetzov \& Stekolnikov, 1978. Ent. Obozr. 57: 871, 872, 879, 881, 885, 887, 888; syn.: Incurvariiformes; infraordem, incluindo Adeloidea.- Kuznetzov \& Stekolnikov, 1979. Ent. Rev. 57: 602, 603, 607, 609, 611, 613; syn.: Incurvariiformes. Nepticulomorpha Kuznetzov \& Stekolnikov, 1978. Ent. Obozr. 57: 871, 872, 879, 882, 888; syn.: Nannolepidoptera;

Revista Brasileira de Zoologia 23 (1): 1-41, março 2006 
infraordem, incluindo Nepticuloidea, Tischerioidea.Kuznetzov \& Stekolnikov, 1979. Ent. Rev. 57: 602, 603, 607, 609, 612, 613; syn.: Nannolepidoptera.

Papilionomorpha Kuznetzov \& Stekolnikov, 1978. Ent. Obozr. 57: 871, 872, 879, 881, 887, 888; syn.: Ditrysia, Distomiogyna; infraordem, incluindo as demais superfamílias.- Kuznetzov \& Stekolnikov, 1978. Ent. Rev. 57: 602, 603, 607, 609, 612, 613; syn.: Ditrysia, Distomiogyna.

Archifrenata Küppers \& Speidel, V-1980. Atalanta 11: 60-63; ramo, incluindo Neopeustidae.- Kristensen \& Nielsen, 1981, in Cederholm. Ent. scand., Suppl. 15: 304.- Nielsen \& Kristensen, 1996. Invert. Tax. 10: 1291.

Eustomatoptera Küppers \& Speidel, 1980. Atalanta 11: 60-63; status não definido, incluindo Archifrenata, Frenatae.Kristensen \& Nielsen, 1981, in Cederholm. Ent. scand., Suppl. 15: 304.

Neopseustina Davis \& Nielsen, 25-XI-1980. Steenstrupia 6: 274; subordem, incluindo Neopseustidae; syn.: Archifrenata.Kristensen \& Nielsen, 1981, in Cederholm. Ent. scand., Suppl. 15: 303.- Kristensen \& Nielsen, 1983. Ztschr. zool. Syst. Evolut.-forsch. 21: 116.- Kristensen, 1984. Steenstrupia 10: 162, 170, 186.- Heppner, 1984. Atlas neotrop. Lep. 2. Checkl. 1, p. XX.- Minet, 1984. Nouv. Rev. Ent. (n. s.) 1: 141, 143, 147.- Nielsen, 1985. Jour. Res. Lep., Suppl. 1: 15.- Minet, 1986. Alexanor 14: 291, 292.Holloway; Bradley \& Carter, 1987. CIE Guides ins. import. man. 1. Lepidoptera, p. 18, 23, 27, 38.- Scoble, 1989, in Emmet \& Head. Moths and Butt. Great Brit. and Ireland 7, p. 15, 17, 21, 22.- Nielsen \& Common, 1991, in CSIRO. Ins. Australia, p. 832, 837.- Scoble, 1992. Lep., p. 203, 205.Heppner, 1995. Atlas neotrop. Lep. 3. Checkl. 2, p. XIII.Heppner, 1996. Atlas neotrop. Lep. 5B. Checkl. 4B, p. XV.Nielsen \& Kristensen, 1996. Invert. Tax. 10: 1200, 1262, 1288, 1293.- Heppner, 1998. Lep. News 1998 (3): $57 .-$ Heppner, 1998. Holarct. Lep. 5, Suppl. 1: 7, 18.- Heppner, 2003. Lep. Florida 1, p. 71; infraordem.

Myoglossata Kristensen \& Nielsen, 1981, in Cederholm. Ent. scand., Suppl. 15: 301, 303; "lineage", incluindo os Lepidoptera com musculatura intrínsica na espirotromba.Davis \& Nielsen, 1980. Steenstrupia 6: 273.- Minet, 1984. Nouv. Rev. Ent. (n. s.) 1: 141.- Kristensen, 1984. Steenstrupia 10: 162, 186; inclue Neopseustina, Exoporia e Heteroneura.- Nielsen, 1985. Jour. Res. Lep., Suppl. 1: 3.Nielsen, 1989, in Fernholm; Bremer \& Jörnvall (Ed.). Hierarchy Life, p. 284, 285.- Scoble, 1989, in Emmet \& Head. Moths and Butt. Great Brit. and Ireland 7: 15, 17, 18, 21.- Nielsen \& Common, 1991, in CSIRO. Ins. Australia, p. 833, 837.- Scoble, 1992. Lep., p. 188.- Poole, 1996, in Poole \& Gentili. Nom. Ins. Nearct. 3, p. 612.- Nielsen \& Kristensen, 1996. Invert. Tax. 10: 1201, 1253, 1262, 1288, 1292, 1294.- Heppner, 1998. Lep. News 1998 (3): 57.Heppner, 1998. Holarct. Lep. 5, Suppl. 1: 7, 18.- Kristensen \& Skalski, 1999, in Kristensen (Ed.), Lep. 1, p. 10, 11, in
Fischer (Ed.). Handb. Zool. 4.- Carter \& Kristensen, 1999, in Kristensen (Ed.), Lep. 1, p. 28, in Fischer (Ed.). Handb. Zool. 4.- Kristensen, 1999, in Kristensen (Ed.), Lep. 1, p. 54, 55, in Fischer (Ed.). Handb. Zool. 4.- Heppner, 2003. Lep. Florida 1, p. 71; coorte e subcoorte.

Heterobathmiina Kristensen, 1983. Ztschr. zool. Syst. Evolut.forsch. 21: 114, 116, 119; subordem, incluindo Heterobathmiidae.- Kristensen, 1984. Steenstrupia 10: 159, 170, 186.- Heppner, 1984. Atlas neotrop. Lep. 2. Checkl. 1, p. XIX.- Minet, 1984. Nouv. Rev. Ent. (n. s.) 1: 147.Nielsen, 1985. Jour. Res. Lep., Suppl. 1: 4, 15.- Minet, 1986. Alexanor 14: 291, 292.- Holloway; Bradley \& Carter, 1987. CIE Guides ins. import. man. 1. Lepidoptera, p. 17, 23, 25, 38.- Nielsen, 1989, in Fernholm; Bremer \& Jörnvall (Ed.). Hierarchy Life, p. 281-284.- Scoble, 1989, in Emmet \& Head. Moths and Butt. Great Brit. and Ireland 7, p. 15, 17, 20.Nielsen \& Common, 1991, in CSIRO. Ins. Australia, p. 831, 833.- Scoble, 1992. Lep., p. 188, 195, 197.- Heppner, 1995. Atlas neotrop. Lep. 3. Checkl. 2., p. XIII.- Heppner, 1998. Lep. News 1998 (3): 57.- Heppner, 1998. Holarct. Lep. 5, Suppl. 1: 7, 18.- Powell et al., 1999, in Kristensen (Ed.), Lep. 1, p. 406, in Fischer (Ed.). Handb. Zool. 4.- Heppner, 2003. Lep. Florida 1, p. 71; subordem.

Apoditrysia Minet, 1983. Ann. Soc. ent. France (n. s.) 19(2): 201; "ensemble", incluindo todos os Ditrysia, menos Tineoidea, Yponomeutoidea, Gelechioidea.- Minet, 1986. Alexanor 14: 294.- Nielsen, 1989, in Fernholm; Bremer \& Jörnvall (Ed.). Hierarchy Life, p. 288; inclue Ditrysia, menos Tineoidea, Yponomeutoidea e Gelechioidea.- Nielsen \& Common, 1991, in CSIRO. Ins. Australia, p. 833.- Minet, 1991. Ent. scand. 22: 71, 72, 80.- Scoble, 1992. Lep., p. 225.- K. Brown, 1996, in Bicudo \& Menezes. Biodiv. Brasil, p. 225.- Poole, 1996, in Poole \& Gentili. Nom. Ins. Nearct. 3, p. 615.- Kristensen \& Skalski, 1999, in Kristensen (Ed.), Lep. 1, p. 10, 13, 14, in Fischer (Ed.). Handb. Zool. 4.- Carter \& Kristensen, 1999, in Kristensen (Ed.), Lep. 1, p. 28, in Fischer (Ed.). Handb. Zool. 4.- Dugdale, 1999, in Kristensen (Ed.), Lep. 1, p. 224, in Fischer (Ed.). Handb. Zool. 4.- Powell et al., 1999, in Kristensen (Ed.), Lep. 1, p. 411, in Fischer (Ed.). Handb. Zool. 4.

Calliduliformes Heppner, 1984. Atlas neotrop. Lep. 2. Checkl. 1, p. XXII; série de Geometroidea, infra-superfamília.

Uraniformes Heppner, 1984. Atlas neotrop. Lep. 2. Checkl. 1, p. XXII; série de Geometroidea, infra-superfamília.

Drepaniformes Heppner, 1984. Atlas neotrop. Lep. 2. Checkl. 1, p. XXII; série de Geometroidea, infra-superfamília.

Saturniiformes Heppner, 1984. Atlas neotrop. Lep. 2. Checkl. 1, p. XXII; série de Bombycoidea, infra-superfamília.Heppner, 1995. Atlas neotrop. Lep. 3. Checkl. 2, p. XIV.Heppner, 1998. Lep. News 1998 (3): 59.- Heppner, 1998. Holarct. Lep. 5, Suppl. 1: 21.

Etimonotrysia Minet, 1984. Nouv. Rev. Ent. (n. s.) 1: 146-148; phalange, incluindo Tischeriidae.- Kristensen, 1985. Ent. Medd. 52: 12.- Minet, 1986. Alexanor 14: 292, 295; syn.: Tischeriina.

Revista Brasileira de Zoologia 23 (1): 1-41, março 2006 
Tischeriina Nielsen, 1985. Jour. Res. Lep., Suppl. 1, p. 16; infraordem, incluindo Tischerioidea.- Kristensen, 1985. Ent. Medd. 52: 9.

Obtectomera Minet, 1986. Alexanor 14: 294; "symplegma", incluindo os Ditrysia superiores: Epermenioidea, Alucitoidea, Copromorphoidea, Thyridoidea, Pyraloidea, Drepanoidea, Axioidea, Mimallonoidea, Bombycoidea, Geometroidea, Calliduloidea, Hesperioidea, Papilionoidea, Uranioidea, Noctuoidea.- Nielsen, 1989, in Fernholm; Bremer \& Jörnvall (Ed.). Hierarchy Life, p. 288; sem status, incluindo mariposas superiores e borboletas.- Nielsen \& Common, 1991, in CSIRO. Ins. Australia, p. 833.- Minet, 1991. Ent. scand. 22: 71, 72, 74, 80, 81.- Scoble, 1992. Lep., p. 225.- de Jong; Vane-Wright \& Ackery, 1996. Ent. scand. 27: 72, 73, 77.- Poole, 1996, in Poole \& Gentili. Nom. Ins. Nearct. 3, p. 617.- Kristensen \& Skalski, 1999, in Kristensen (Ed.), Lep. 1, p. 10, 12, 14, in Fischer (Ed.). Handb. Zool. 4.- Carter \& Kristensen, 1999, in Kristensen (Ed.), Lep. 1, p. 28, in Fischer (Ed.). Handb. Zool. 4.- Dugdale et al., 1999, in Kristensen (Ed.), Lep. 1, p. 224, in Fischer (Ed.). Handb. Zool. 4.- Powell et al., 1999, in Kristensen (Ed.), Lep. 1, p. 412, in Fischer (Ed.). Handb. Zool. 4.

Lophocoronina Nielsen \& Common, 1991, in CSIRO. Ins. Australia, p. 832, 837; subordem, incluindo Lophocoronidae.Scoble, 1992. Lep., p. 205.- Heppner, 1995. Atlas neotrop. Lep. 3. Checkl. 2, p. XIII.- Heppner, 1996. Atlas neotrop. Lep. 5B. Checkl. 4B, p. XV; infraordem.- Nielsen \& Kristensen, 1996. Invert. Tax. 10: 1293.- Heppner, 1998. Lep. News 1998 (3): 57.- Heppner, 1998. Holarct. Lep. 5, Suppl. 1: 7, 18.- Heppner, 2003. Lep. Florida 1, p. 71; infraordem

Sesiina Heppner, 1995. Atlas neotrop. Lep. 3. Checkl. 2, p. XIII; subseção, incluindo Sesioidea e Zygaenoidea.- Heppner, 1996. Atlas neotrop. Lep. 5B. Checkl. 4B, p. XV.- Heppner, 1998. Lep. News 1998 (3): 58.- Heppner, 1998. Holarct. Lep. 5, Suppl. 1: 7, 20.- Heppner, 2003. Lep. Florida 1, p. 72, 92, 94, 308; subseção.

Coelolepida Nielsen \& Kristensen, 1996. Invert. Tax. 10: 1293, 1294; clado que inclue Acanthopteroctetidae + Lophocoronidae + Myoglossata.- Kristensen \& Skalski, 1999, in Kristensen (Ed.), Lep. 1, p. 10, 11, in Fischer (Ed.). Handb. Zool. 4.- Carter \& Kristensen, 1999, in Kristensen (Ed.), Lep. 1, p. 28, in Fischer (Ed.). Handb. Zool. 4.- Kristensen, 1999, in Kristensen (Ed.), Lep. 1, p. 53, in Fischer (Ed.). Handb. Zool. 4.

Ditrisia [sic]; K. Brown, 1996, in Bicudo \& Menezes. Biodiv. Brasil, p. 224.

Rophalocera [sic]; Prieto \& Constantino, 1996. Bol. Mus. Ent. Univ. Valle, Cali, 4(2): 11-18.- Parra; Vargas \& Tabares, 2000. Marip. Manizales, p. 22.

Gracillariiformes Heppner, IX-1998. Lep. News 1998 (3): 58; série, incluindo as seguintes famílias de Tineoidea: Amphitheridae, Schreckensteiniidae, Douglasiidae, Bucculatricidae, Gracillariidae, infra-superfamília.- Heppner, XI-1998. Holarct. Lep. 5, Suppl. 1: 19.
Limacodiformes Heppner, IX-1998. Lep. News 1998 (3): 58; série, incluindo as seguintes famílias de Cossoidea: Cyclotornidae, Epipyrodidae, Dalceridae, Limacodidae, Chrysopolomidae, infrasuperfamília.- Heppner, XI-1998. Holarct. Lep. 5, Suppl. 1: 20.

\section{ESQUEMA FILOGENÉTICO DA ORDEM LEPIDOPTERA CONFORME HEPPNER (2003)}

O esquema filogenético proposto por HeppNer (2003) é fornecido na tabela I.

\section{LISTA SINONIIMICA DOS NOMES DOS TAXA SUPERIORES}

A publicação mais recente com uma proposta de classificação filogenética para Lepidoptera é de HeppNer (2003) (vide item II), razão pela qual serviu como base para o estabelecimento dos sinônimos. Em outra recente publicação, a única mudança neste esquema filogenético foi aceitar a superfamília Hesperioidea, como grupo irmão de Papilionoidea (Lamas 2004, in Heppner (Ed.)).

Muitos taxa são de difícil interpretação, e neste caso não sinonimizados, no entanto, estão listados, já que podem se úteis em estudos posteriores. A lista que segue inclui os nomes com os sinônimos que foram possíveis de estabelecer.

\section{Superordem Lepidopteroidea Heymons, 1915. nom. rev.}

Sinônimos: Amphiesmenoptera Kiriakoff, 1948, syn. nov.; Amphiesmenoptères [sic]; Kiriakoff, 1952.

\section{Ordem Lepidoptera Linnaeus, 1758}

Sinônimos: Tetraptera alis farinaceis Geoffroy, 1762; Farinacea Schluga, 1767; Farinosa Schluga, 1767; Papilionoides Laicharting, 1781; Lépidoptères [sic] Latreille, 1796; Lepidioptera [sic]; Clairville, 1798; Lepidopteria [sic]; Rafinesque, 1815; Glossostoma Leach, 1815; Alis farinaceis Kirby \& Spence, 1826; Micrognatha Spinola, 1852; Lepidopters [sic]; Dana, 1864; Sorbentia Haeckel, 1889.

Subordem Micropterygina Herrich-Schäffer, 1856. nom. rev.

Sinônimos: Microjugatae J. H. Comstock, 1893; Laciniata Packard,. 1895; Protolepidoptera Packard, 1895; Zeugloptera Chapman, 1916, syn. nov.; Zeugloptères [sic]; Kiriakoff, 1952; Micropterigomorpha Kuznetzov \& Stekolnikov, 1978;

\section{Subordem Aglossata Speidel, 1977}

Sinônimos: Não há.

\section{Subordem Heterobathmiina Kristensen, 1983}

Sinônimos: Não há.

Subordem Glossata Fabricius, 1775

Sinônimo: Glossates [sic]; Duméril, 1806

Coorte Paleolepidoptera Packard, 1895. nom. rev.

Sinônimos: Dacnonypha Hinton, 1946, syn. nov.; Hoplos- 
Tabela I. Esquema de classificação de Lepidoptera, segundo HeppNER (2003). Superordem Lepidopteroidea Heymons, 1925; Ordem Lepidoptera Linnaeus, 1758

\begin{tabular}{|c|c|c|c|c|c|c|c|}
\hline Subordem & Coorte & Subcoorte & Infraordem & Divisão & Seção & Subseção & Superfamília \\
\hline Zeuglopterea & & & & & & & 1 - Micropterigoidea \\
\hline Aglossata & & & & & & & 2 - Agathiphagoidea \\
\hline Heterobathmiina & & & & & & & 3 - Heterobathmioidea \\
\hline \multirow[t]{30}{*}{ Glossata } & Dacnonypha & & Dacnonypha & & & & 4 - Eriocranoidea \\
\hline & & & Lophocoronina & & & & 5 -Lophocoronoidea \\
\hline & Myoglossata & Myoglossata & Neopseutina & & & & 6 - Neopseustoidea \\
\hline & & Neolepidoptera & Exoporia & & & & 7 - Mnesarchaeoidea \\
\hline & & & Heteroneura & Monotrysia & Nepticulina & & 8 - Andesianoidea \\
\hline & & & & & & & 10 - Nepticuloidea \\
\hline & & & & & & & 11 - Tischerioidea \\
\hline & & & & & & & 12 - Palaephatoidea \\
\hline & & & & & Incurvariina & & 13 - Incurvarioidea \\
\hline & & & & Ditrysia & Tineina & Tineina & 14 - Tineoidea \\
\hline & & & & & & & 15 - Gelechioidea \\
\hline & & & & & & & 16 - Copromorphoidea \\
\hline & & & & & & & 17 - Yponomeutoidea \\
\hline & & & & & & & 18 - Immoidea \\
\hline & & & & & & & 19 - Pyraloidea \\
\hline & & & & & & & 20 - Pterophoroidea \\
\hline & & & & & & Sesiina & 21 - Sesioidea \\
\hline & & & & & & & 22 - Zygaenoidea \\
\hline & & & & & Cossina & Cossina & 23 - Cossoidea \\
\hline & & & & & & & 24 - Castnioidea \\
\hline & & & & & & & 25 - Tortricoidea \\
\hline & & & & & & Bombycina & 26 - Calliduloidea \\
\hline & & & & & & & 27 - Uranioidea \\
\hline & & & & & & & 28 - Geometroidea \\
\hline & & & & & & & 29 - Papilionoidea \\
\hline & & & & & & & 30 - [Hesperioidea] \\
\hline & & & & & & & 31 - Drepanoidea \\
\hline & & & & & & & 32 - Bombycoidea \\
\hline & & & & & & & 33 - Sphingoidea \\
\hline & & & & & & & 34 - Noctuoidea \\
\hline
\end{tabular}

tomatoptera Kiriakoff, 1948; Hoplostomatoptères [sic]; Kiriakoff; 1952; Dachnonypha [sic]; Common, 1970; Eriocraniomorpha Kuznetzov \& Stekolnikov, 1978.

Infraordem Paleolepidoptera Packard, 1895. nom. rev. Sinônimos: Não há.

Infraordem Lophocoronina Nielsen \& Common, 1991 Sinônimos: Não há.

\section{Coorte Myoglossata Kristensen \& Nielsen, 1981} Sinônimos: Não há.
Subcoorte Myoglossata Kristensen \& Nielsen, 1981 Sinônimos: Não há.

Infraordem Archifrenata Küppers \& Speidel,V-1980. nom. rev.

Sinônimo: Neopseustina Davis \& Nielsen, XI-1980, syn. nov.

Subcoorte Neolepidoptera Packard, 1895 Sinônimos: Não há.

Infraordem Hepialites Latreille, 1829. nom. rev.

Sinônimos: Infrenatae Guenée, 1875; Terricolae Guenée,

Revista Brasileira de Zoologia 23 (1): 1-41, março 2006 
Tabela II. Esquema de classificação de Lepidoptera, segundo HEPPNER (2003), com a inclusão dos sinônimos estabelecidos. Superordem Lepidopteroidea Heymons, 1925; Ordem Lepidoptera Linnaeus, 1758

\begin{tabular}{|c|c|c|c|c|c|c|c|}
\hline Subordem & Coorte & Subcoorte & Infraordem & Divisão & Seção & Subseção & Superfamília \\
\hline Micropterygina & & & & & & & 1 - Micropterigoidea \\
\hline Aglossata & & & & & & & 2 - Agathiphagoidea \\
\hline Heterobathmiina & & & & & & & 3 - Heterobathmioidea \\
\hline \multirow[t]{30}{*}{ Glossata } & Paleolepidoptera & & Paleolepidoptera & & & & 4 - Eriocranoidea \\
\hline & & & Lophocoronina & & & & 5 -Lophocoronoidea \\
\hline & Myoglossata & Myoglossata & Archifrenata & & & & 6 - Neopseustoidea \\
\hline & & Neolepidoptera & Hepialites & & & & 7 - Mnesarchaeoidea \\
\hline & & & Anisoneuria & Monotrysia & Nepticulae & & 8 - Andesianoidea \\
\hline & & & & & & & 10 - Nepticuloidea \\
\hline & & & & & & & 11 - Tischerioidea \\
\hline & & & & & & & 12 - Palaephatoidea \\
\hline & & & & & Adeliformes & & 13 - Incurvarioidea \\
\hline & & & & Ditrysia & Tineae & Tineae & 14 - Tineoidea \\
\hline & & & & & & & 15 - Gelechioidea \\
\hline & & & & & & & 16 - Copromorphoidea \\
\hline & & & & & & & 17 - Yponomeutoidea \\
\hline & & & & & & & 18 - Immoidea \\
\hline & & & & & & & 19 - Pyraloidea \\
\hline & & & & & & & 20 - Pterophoroidea \\
\hline & & & & & & Zygaenides & 21 - Sesioidea \\
\hline & & & & & & & 22 - Zygaenoidea \\
\hline & & & & & Cossina & Cossina & 23 - Cossoidea \\
\hline & & & & & & & 24 - Castnioidea \\
\hline & & & & & & & 25 - Tortricoidea \\
\hline & & & & & & Bombyces & 26 - Calliduloidea \\
\hline & & & & & & & 27 - Uranioidea \\
\hline & & & & & & & 28 - Geometroidea \\
\hline & & & & & & & 29 - Papilionoidea \\
\hline & & & & & & & 30 - [Hesperioidea] \\
\hline & & & & & & & 31 - Drepanoidea \\
\hline & & & & & & & 32 - Bombycoidea \\
\hline & & & & & & & 33 - Sphingoidea \\
\hline & & & & & & & 34 - Noctuoidea \\
\hline
\end{tabular}

1875; Macrojugatae J. H. Comstock, 1893; Aplostomatoptera Kiriakoff, 1948, Aplostomatoptères [sic]; Kiriakoff, 1952; Gymnocera Friese, 1970; Exoporian Ditrysia Dugdale, 1974, Exoporia Common, 1975, syn. nov.; Hepialomorpha Kuznetzov \& Stekolnikov, 1978; Aplostommatoptera [sic]; Gómez B., 1978.

\section{Infraordem Anisoneuria Karsch, 1898. nom. rev.}

Sinônimos: Heteroneura Tillyard, 1918, syn. nov.; Heteroneures [sic]; Lameere, 1938.

\section{Divisão Monotrysia Börner, 1925}

Sinônimo: Não há.

\section{Seção Nepticulae Chapman, 1893. nom. rev.}

Sinônimos: Nepticulina Meyrick, 1928, syn. nov.; Nannolepidoptera Kiriakoff, 1948; Nannolepidoptères [sic]; Kiriakoff, 1952; Nennolepidoptera [sic]; Razowski, 1974; Nepticulomorpha Kuznetzov \& Stekolnikov, 1978.

\section{Seção Adeliformes Lameere, 1936. nom. nov.}

Sinônimos: Tineides aculeatae Spuler, 1910 (binominal); Incurvariina Börner, 1939, syn. nov.; Stigmellina Le Marchand, 1945; Tineaea aculeatae [sic]; Kiriakoff, 1946; Incurvariiformes Kiriakoff, 1948. 


\section{Divisão Ditrysia Börner, 1925}

Sinônimos: Distomiogyna Le Marchand, 1945; Endoporian Ditrysia Dugdale, 1974; Ditrisia [sic]; K. Brown, 1996.

\section{Seção Tineae Scopoli, 1763. nom. rev.}

Sinônimos: Tineites Latreille, 1810; Tineides Leach, 1815; Teignes Latreille, 1817; Tineina Stainton, 1857, syn. nov.; Tineoids Chapman, 1893; Stenopterygia Packard, 1895; Tineaemorpha Krausse \& Wolff, 1919; Tineifromes Lameere, 1936.

Subseção Tineae Scopoli, 1763. nom. rev.

Sinônimo: Não há.

\section{Subseção Zygaenides Leach, 1815. nom. rev.}

Sinônimos: Sésiens Blanchard, 1845; Zygaeniens Blanchard, 1845; Spinicornes Guenée, 1875; Zygaenomorpha Krausse \& Wolff, 1919; Zygéniformes Lameere, 1936; Sesiina Heppner, 1995, syn. nov.

\section{Seção Cossina Herrich-Schäffer, 1856}

Sinônimo: Cossiformes Lameere, 1936.

Subseção Cossina Herrich-Schäfer, 1856

Sinônimo: Lignivorae Guenée, 1875.

\section{Subseção Bombyces Scopoli, 1763. nom. rev.}

Sinônimos: Bombycites Latreille, 1809; Bombycides Leach, 1815; Bombyciens Blanchard, 1845; Bombyciformes Guenée, 1852; Saturniina Herrich-Schäffer, 1856; Bombycina Stainton, 1857, syn. nov.; Stellatae Guenée, 1875; Saturnia Dyar, 1895; Saturnina Dyar, 1895; Bombycimorpha Krausse \& Wolff, 1919; Saturniiformes Heppner, 1984.

\section{ESQUEMA FILOGENÉTICO DA ORDEM LEPIDOPTERA, BASEADO EM HEPPNER (2003) COM INCLUSÃO DOS SINÔNIMOS AQUI ESTABELECIDOS}

O esquema filogenético proposto por HePpNer (2003) considerando-se a inclusão dos sinônimos estabelecidos no presente trabalho é fornecido na tabela II.

\section{ÍNDICE DOS NOMES DOS TAXA SUPERIORES}

Acalinopteros

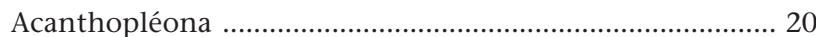

Achalinoptera …................................................................ 13

Achalinoptères .................................................................... 10

Acraeomorpha .................................................................... 15

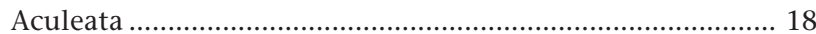

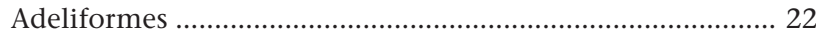

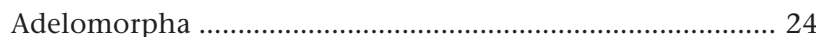

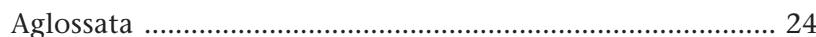

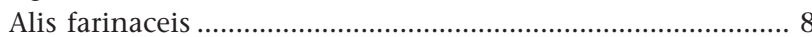

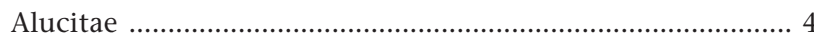

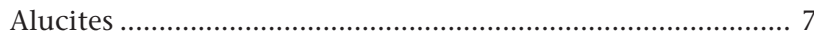

Alucitides …...................................................................... 7

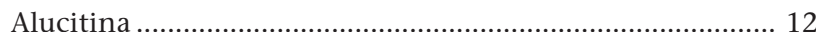

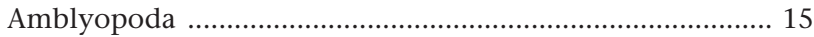

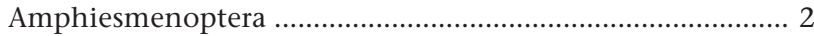

Amphiesménoptères ................................................................... 2

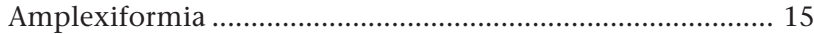

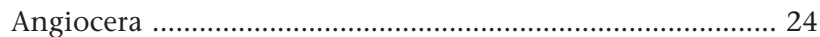

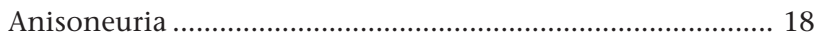

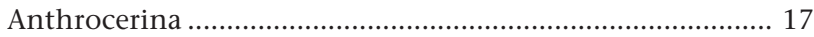

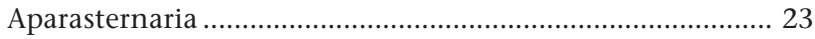

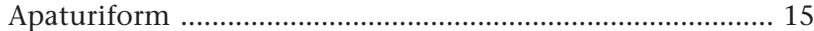

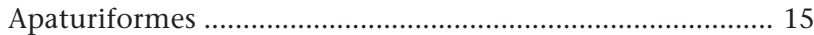

Aplostomatoptera …........................................................... 23

Aplostomatoptères ............................................................... 23

Aplostommatoptera .............................................................. 24

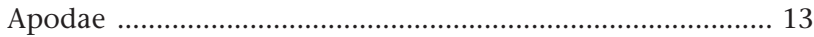

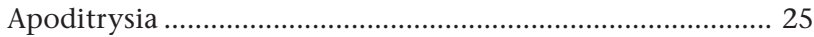

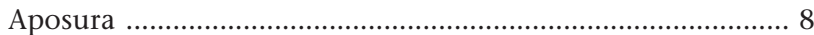

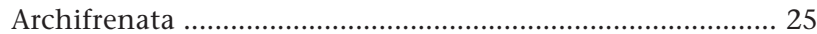

Archilepidoptera ............................................................... 18

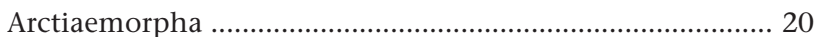

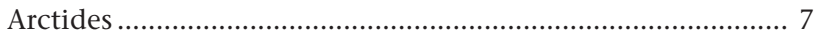

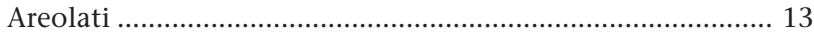

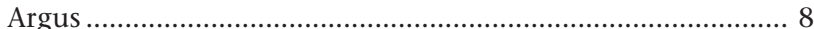

Arpenteuses ....................................................................... 7

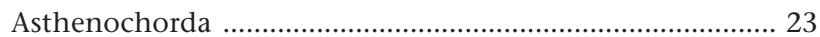

Bicalcarati ........................................................................... 13

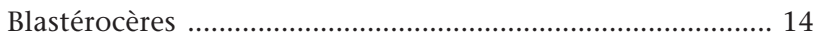

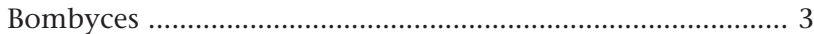

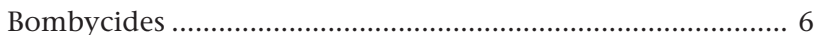

Bombyciens ....................................................................... 10

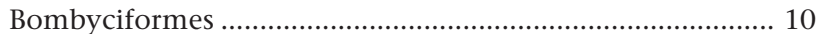

Bombycimorpha ............................................................... 20

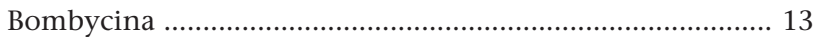

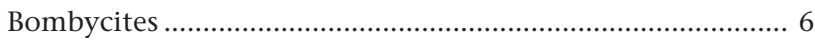

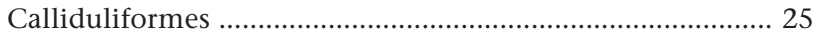

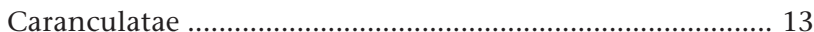

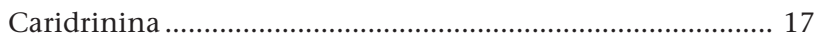

Castniens

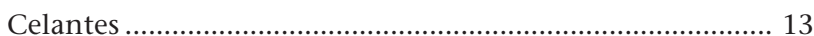

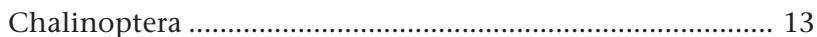

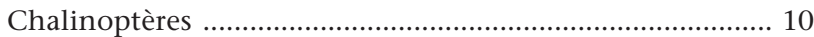

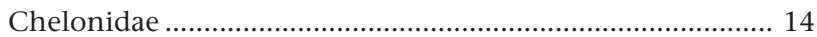

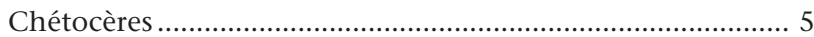

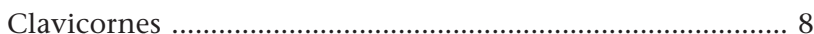

Clostérocères ……………………………………………..... 5

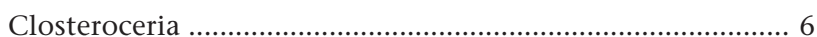

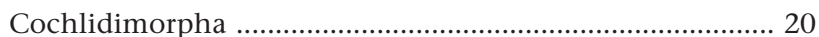

Coelolepida …................................................................. 26

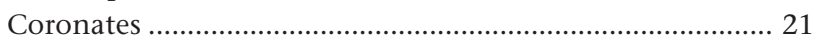

Coronofrenatae .................................................................. 19

Cossiformes ....................................................................... 22

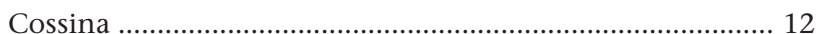

Crambidae ………………………………………………... 14

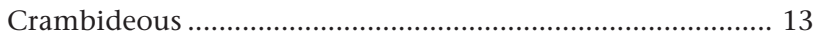

Revista Brasileira de Zoologia 23 (1): 1-41, março 2006 


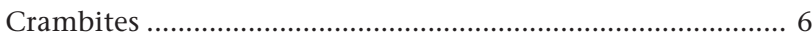

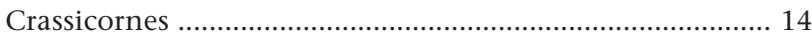

Crepusculaires .................................................................. 10

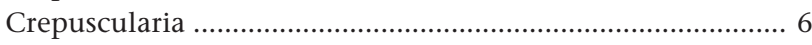

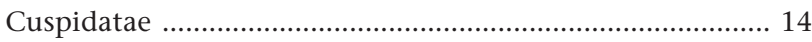

Cydemonii ....................................................................... 13

Cydemoniens ........................................................................ 10

Cylindracei ......................................................................... 13

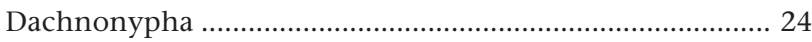

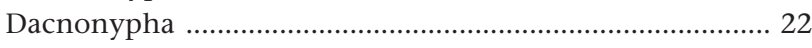

Danaiform ...................................................................... 14

Danaiformes ..................................................................... 15

Danaomorpha .................................................................. 15

Deltoidae ........................................................................... 14

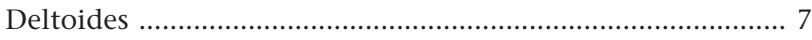

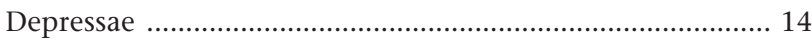

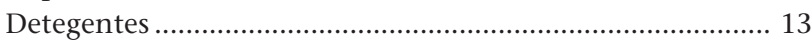

Distomiogyna ...................................................................... 22

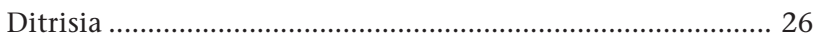

Ditrysia ....................................................................... 21

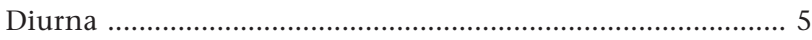

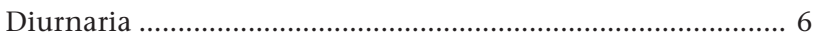

Diurnes ............................................................................. 4

Diurni ............................................................................... 5

Drepaniformes ..................................................................... 25

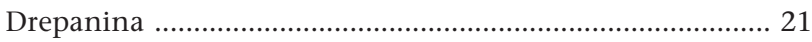

Elongatae ........................................................................... 14

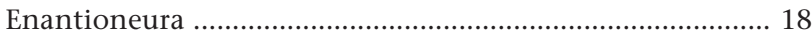

Endophytes ........................................................................ 14

Endoporian Ditrysia ............................................................... 24

Ephemerina ….............................................................. 7

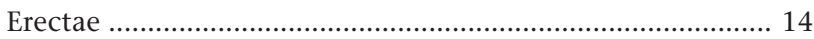

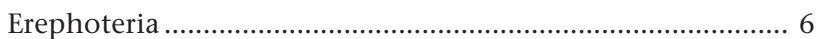

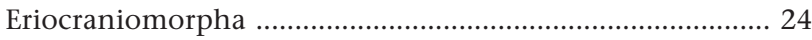

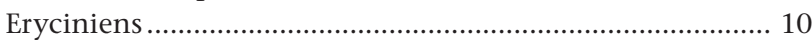

Etimonotrysia …................................................................... 25

Eulepidoptera ...................................................................... 22

Eulépidoptères ......................................................................... 23

Eustomatoptera (............................................................... 25

Exoporia ...................................................................... 24

Exoporian Ditrysia ............................................................. 24

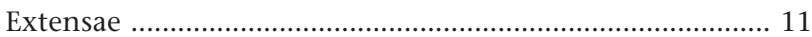

Farinacea …...................................................................... 4

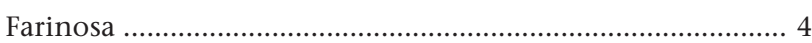

Filicornes …….................................................................... 5

Fimbriatae ...................................................................... 14

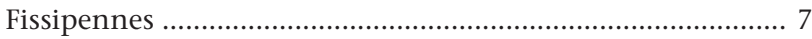

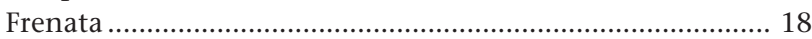

Frenatae ....................................................................... 15

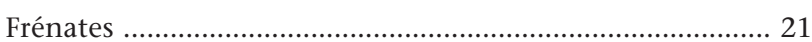

Frenati ............................................................................. 20

Frenulum-conservers ......................................................... 17

Frenulum-losers ........................................................... 17
Furculae ............................................................................... 13

Fusicornes ….................................................................. 5

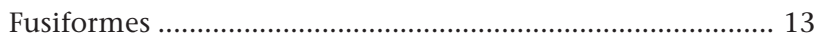

Gelechiiformes ................................................................... 23

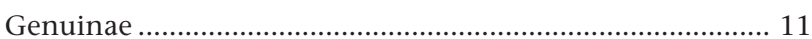

Geometrae ....................................................................... 3

Geometraemorpha ............................................................. 20

Geometriformes .................................................................... 22

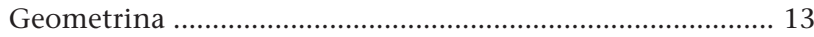

Globulicornes ..................................................................... 5

Globulosae ....................................................................... 14

Glossata ....................................................................... 4

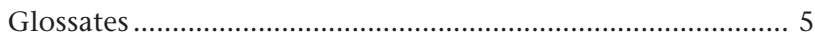

Glossostoma .................................................................... 6

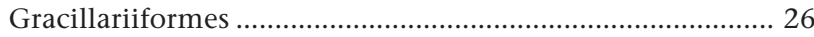

Granulosae ............................................................................ 13

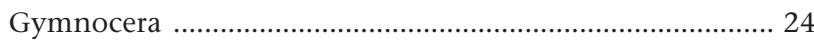

Harmoncopoda ................................................................. 18

Haustellata ....................................................................... 4

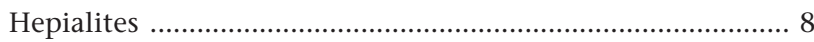

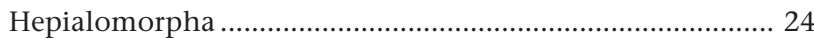

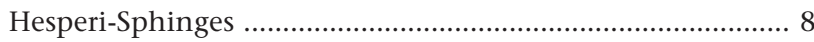

Heterobathmiina .................................................................... 25

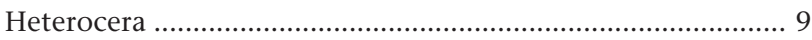

Heterocères .......................................................................... 9

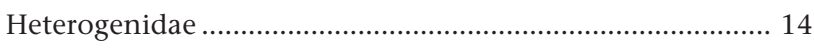

Heteroneura ........................................................................ 19

Heteroneures ..................................................................... 22

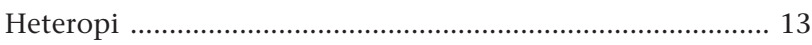

Heteropoda ........................................................................ 15

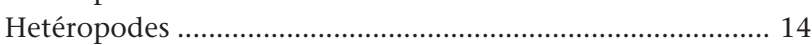

Heterotrysia _......................................................................... 22

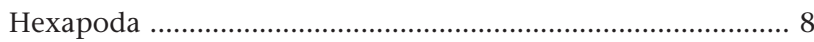

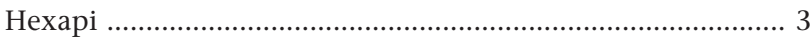

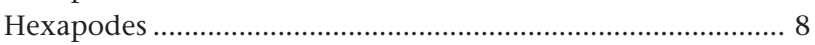

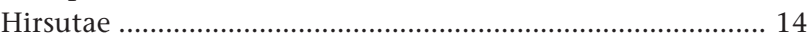

Holopoda ......................................................................... 21

Homoiopoda ...................................................................... 15

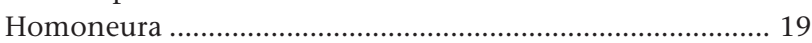

Homoneures ......................................................................... 22

Hoplostomatoptera .......................................................... 23

Hoplostomatoptères ............................................................ 23

Inclinatae ........................................................................ 14

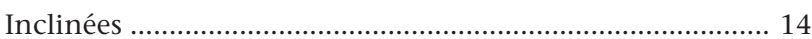

Incompletae ..................................................................... 16

Incurvariiformes ............................................................... 23

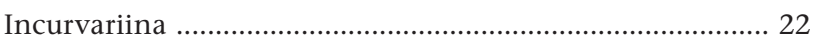

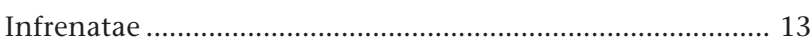

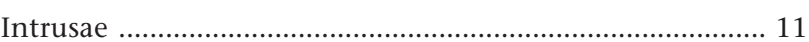

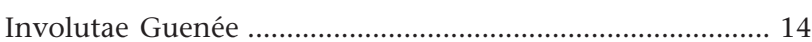

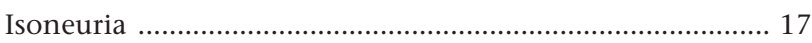

Jugata ............................................................................ 18

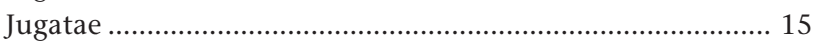




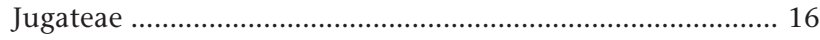

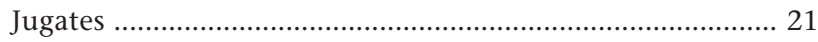

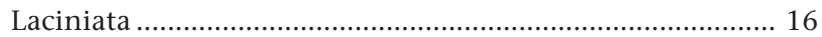

Lasiocampina .................................................................... 12

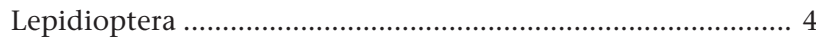

Lepidoptera ..................................................................... 2

Lépidoptères .......................................................................... 4

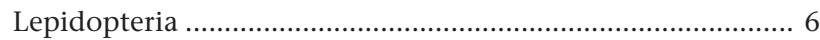

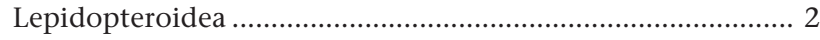

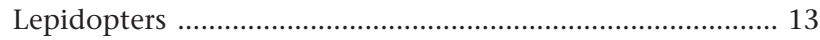

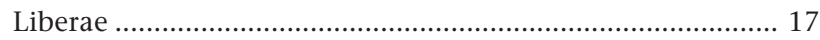

Lichenivorae ................................................................... 14

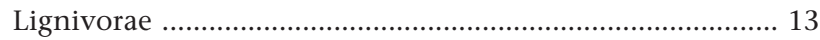

Limaciformes ..................................................................... 13

Limacodiformes ................................................................ 26

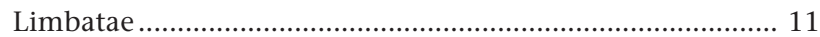

Lipacánthina ........................................................................ 20

Lophocoronina …............................................................. 26

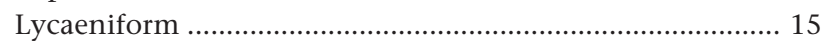

Lycaeniformes ................................................................. 15

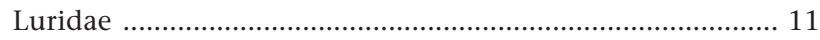

Macrofrenatae ..................................................................... 16

Macroheterocera ................................................................... 16

Macro-Heterocera .................................................................. 16

Macrojugatae ...................................................................... 16

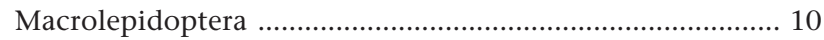

Macrolépidoptères ................................................................. 22

Macróptera .......................................................................... 20

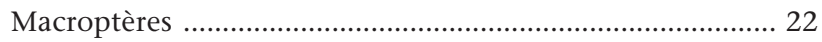

Macros ….......................................................................... 16

Makrolepidoptera …........................................................... 14

Microfrenatae ..................................................................... 16

Micrognatha ..................................................................... 10

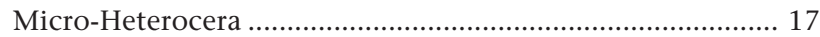

Microjugatae .................................................................. 16

Microlepidoptera ............................................................. 9

Microlepidopteren ............................................................. 9

Microlépidoptères ................................................................... 22

Microlopidopteren …........................................................... 10

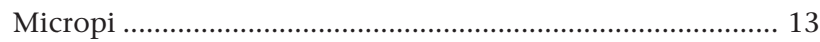

Micros ……................................................................. 16

Micróptera ..................................................................... 20

Microptères ……............................................................ 22

Micropterigomorpha .......................................................... 24

Micropterygina .................................................................. 12

Microptila ....................................................................... 23

Mikrolepidoptera .............................................................. 14

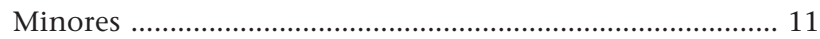

Monostomiogyna ................................................................ 22

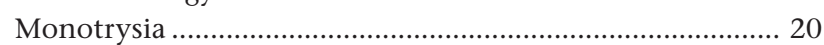

Myoglossata ........................................................................... 25

Nannolepidoptera .............................................................. 23

Nannolepidoptères ............................................................ 23
Nématocères ................................................................................ 5

Nennolepidoptera ............................................................. 24

Neolepidoptera ................................................................ 17

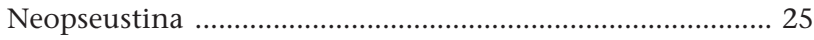

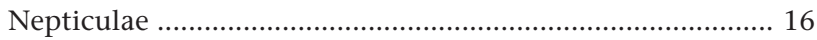

Nepticulina ....................................................................... 21

Nepticulomorpha ................................................................ 24

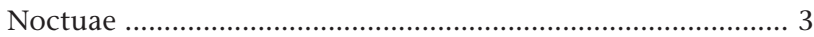

Noctuaelites .......................................................................... 6

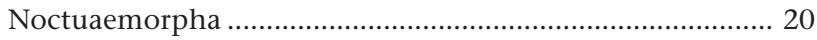

Noctuéliens ......................................................................... 10

Noctuélites ........................................................................ 10

Noctuides .......................................................................... 7

Noctuiformes ..................................................................... 22

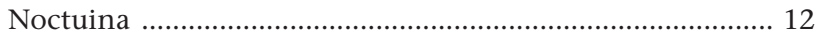

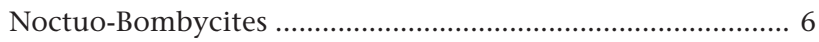

Noctuo-Hepialid ............................................................ 17

Nocturna ....................................................................... 6

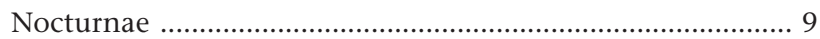

Nocturnaria ........................................................................ 6

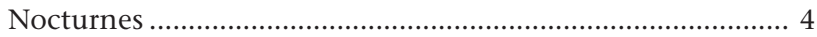

Nocturni ................................................................................ 5

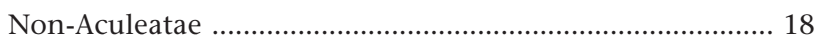

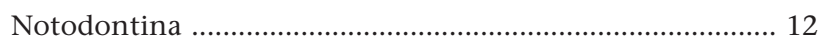

Nude ..................................................................... 14

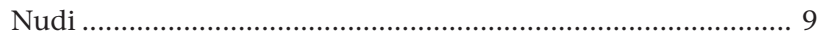

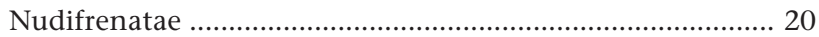

Nymphaliens ................................................................... 10

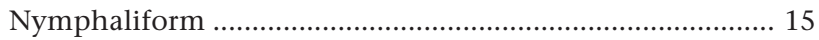

Nymphaliformes ................................................................... 15

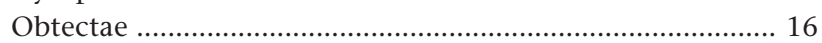

Obtectomera ................................................................... 26

Onisciformes .................................................................. 13

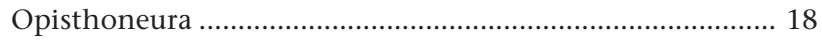

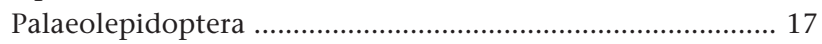

Paleohesperidae ..................................................................... 15

Paleolepidoptera ................................................................. 17

Pannosae ............................................................................ 14

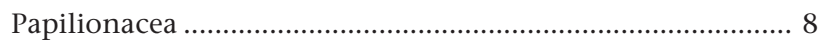

Papilionides ...................................................................... 5

Papilioniens ................................................................... 10

Papilioniformes ............................................................... 22

Papilionina ...................................................................... 9

Papilionoides ..................................................................... 4

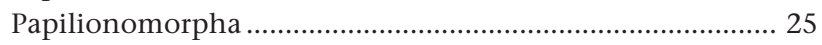

Papillonides ...................................................................... 8

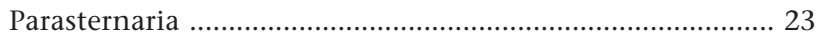

Parnassi-Papilionidae ....................................................... 17

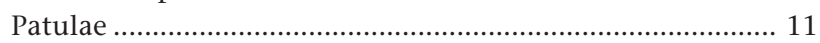

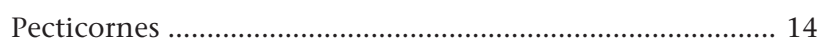

Pedunculata ……........................................................... 13

Pellucidae ................................................................................ 14

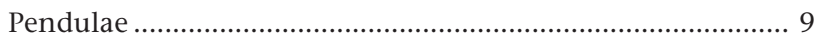


Pendulae B

Penduli

Perlata.....

Peropoda

Perpendiculaires

Phalaenae Tortices .............................................................. 8

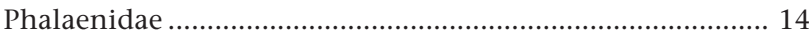

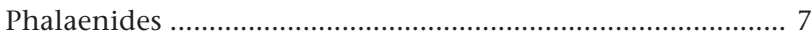

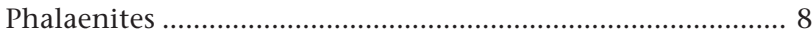

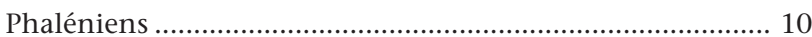

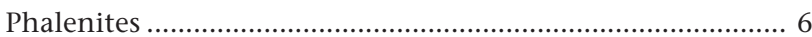

Phycidae ......................................................................... 14

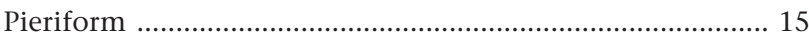

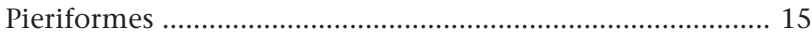

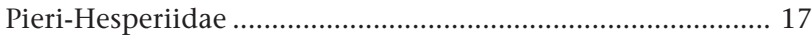

Plantivorae ................................................................................ 14

Platylepidoptera ................................................................ 17

Platyptères .......................................................................... 22

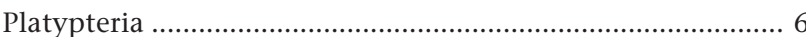

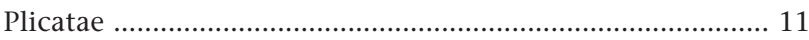

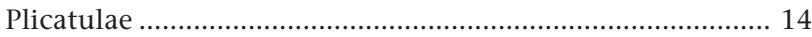

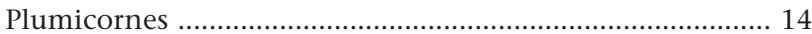

Pomeridiana …................................................................... 8

Prismaticornes ..................................................................... 13

Protolepidoptera .................................................................. 17

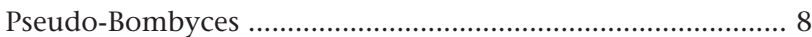

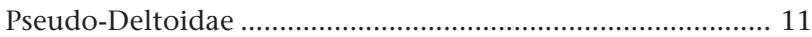

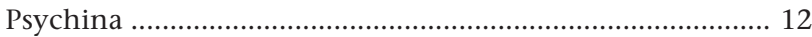

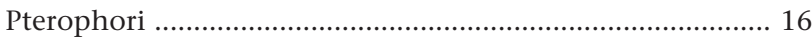

Pterophorina .......................................................................... 12

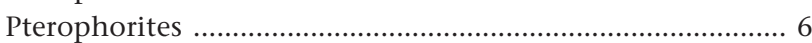

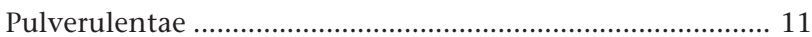

Pyrales .............................................................................. 4

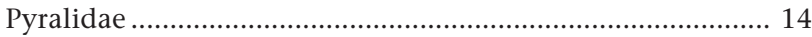

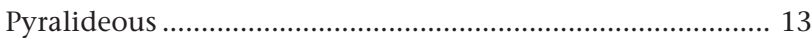

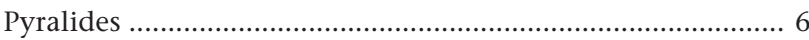

Pyralidiformes ………...................................................... 23

Pyralidina ............................................................................ 12

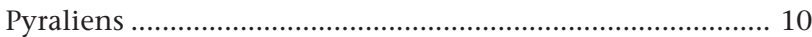

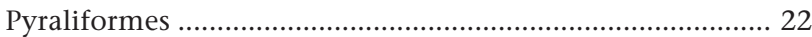

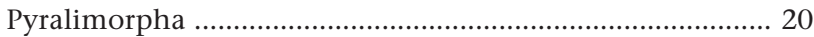

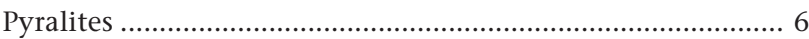

Pyralo-Micropterygid ............................................................ 17

Quadrifidae ................................................................ 11

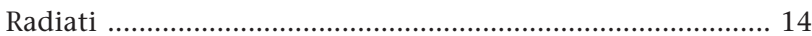

Rhopalocera ....................................................................... 7

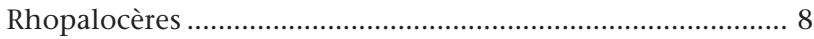

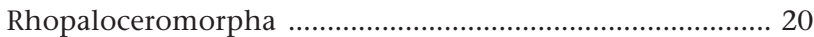

Ropalocères .......................................................................... 5

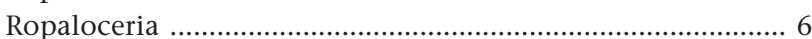

Rophalocera ......................................................................... 26

Saccophorae ....................................................................... 14

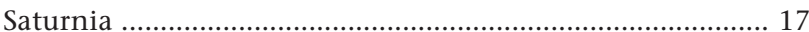

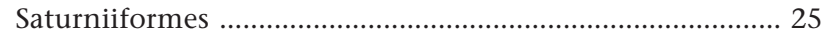

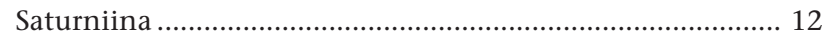

Saturnina ...................................................................... 16

Satyriform ….................................................................. 15

Satyriformes .................................................................... 15

Satyromorpha …........................................................... 15

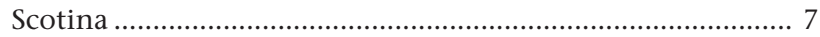

Sémicoronates ..................................................................... 22

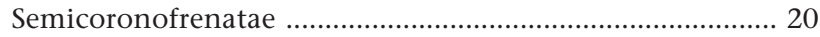

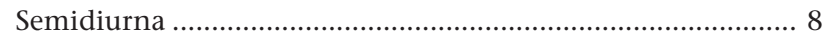

Sericeae .......................................................................... 11

Serpentinae …................................................................ 11

Sésiens .......................................................................... 10

Sesiina ........................................................................ 26

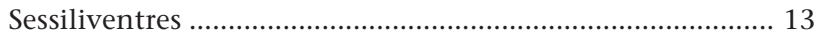

Séticornes ......................................................................... 5

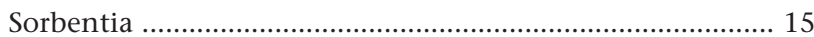

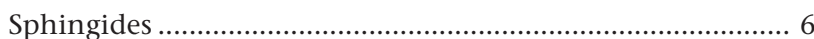

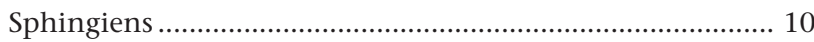

Sphingiformes ................................................................... 23

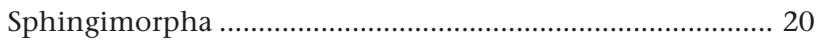

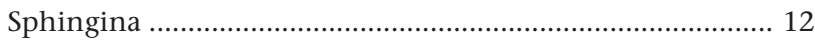

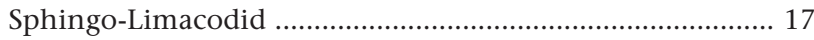

Sphingo-Micropterygides ................................................... 18

Spinicornes .................................................................... 13

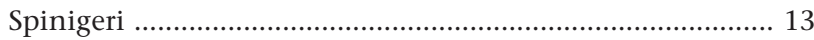

Spinosae .......................................................................... 13

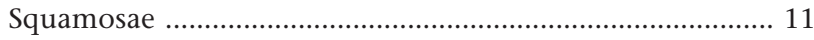

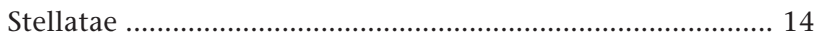

Stemmantoncopoda ............................................................. 18

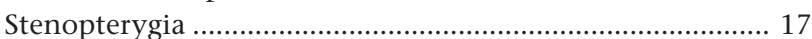

Sthenochorda .................................................................... 23

Stigmellina .................................................................... 22

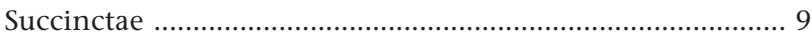

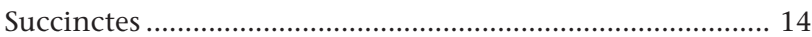

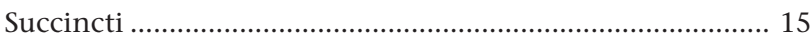

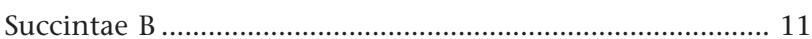

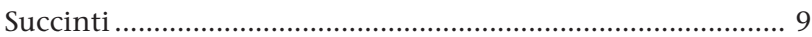

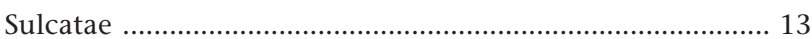

Suspendues ...................................................................... 14

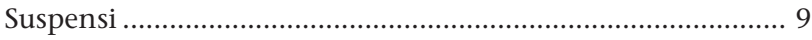

Synemonii ........................................................................ 13

Teignes ................................................................................. 7

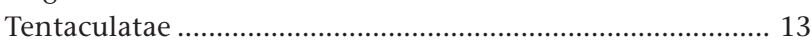

Terricolae ................................................................................ 13

Tetrapi …......................................................................... 3

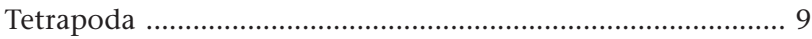

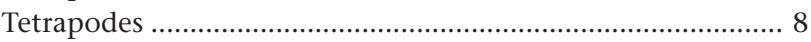

Tetraptera alis farinaceis .......................................................... 3

Tineae ............................................................................ 4

Tineaea aculeatae .............................................................. 22

Tineaemorpha .................................................................... 19

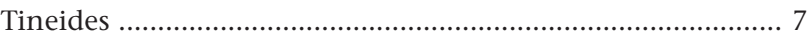


Tineides aculeatae ............................................................. 18

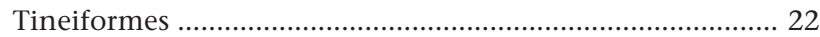

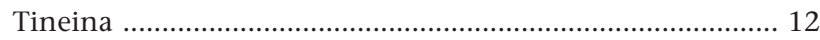

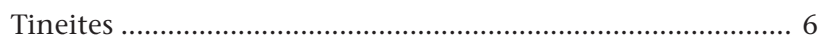

Tineoids ......................................................................... 16

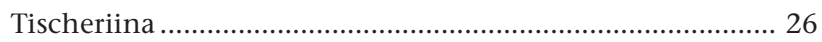

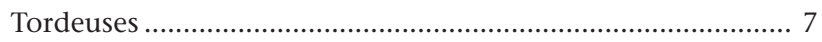

Tortrices …......................................................................... 3

Tortriciformes ........................................................................ 14

Tortricimorpha ................................................................ 20

Tortricina ....................................................................... 13

Tortricoids ....................................................................... 16

Trifidae ...................................................................... 10

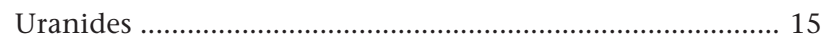

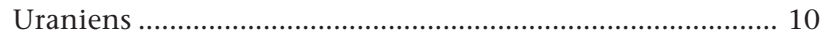

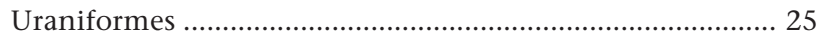

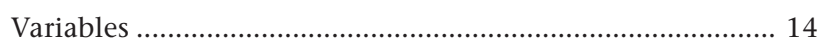

Variegatae .................................................................... 11

Verrucosae ....................................................................... 14

Verticillatae ...................................................................... 14

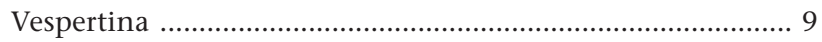

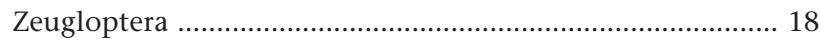

Zeugloptères ............................................................................. 23

Zygaenaemorpha ............................................................... 20

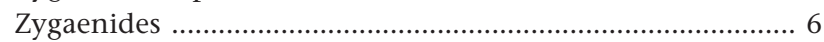

Zygaeniens .................................................................... 10

Zygéniformes .................................................................... 22

\section{AGRADECIMENTOS}

Agradecemos aos seguintes colegas que gentilmente nos receberam e auxiliaram na procura das bibliografias necessárias à elaboração desta lista: Drs. Richard I. Vane-Wright, Philipp Ronald Ackery (The Natural History Musem, Londres, Inglaterra), Wolfram Mey (Naturkunde Museum der Humboldt Universität, Berlin, Alemanha), Georges Bernardi, Jacques Pierre (Muséum national d'Histoire naturelle, Paris, França), Frederick Rindge e James S. Miller (American Museum of Natural History, New York, USA), Robert K. Robbins, John Burns e Donald James Harvey (National Museum of Natural History, Smithsonian Institution, Washington, D.C., USA) e Gerardo Lamas (Museu de Historia Natural, Lima, Peru).

\section{REFERÊNCIAS BIBLIOGRÁFICAS}

Acloque, A. 1897. Faune de France, contenant la description des espèces indigènes disposées en tableaux analytiques et illustrée de figures représentant les types caractéristiques des genres. 2. Orthoptères, Névroptères, Hymenoptères, Lépidoptères, Hemiptères, Diptères, Aphaniptères, Thysanoptères, Rhipiptères. Paris, Libr. J.-B. Baillières \& Fils, VII+516p.

Agenjo, R. 1946. Catalogo ordenador de los lepidopteros de España. Graellsia, Madri, 4 (3): [1-11]; 5(1): [1-15].
Aurivillius, C. 1925. Die afrikanischen Tagfalter. In: A. SeITZ. Die Gross-Schmetterlinge der Erde 13. Die afrikanischen Tagfalter. Stuttgart, Alfred Kernen Verlag, VII+612p.

$\mathrm{BAR}, \mathrm{C}$. 1878. Note critique sur les différent systèmes de classification des lépidoptères rhopalocères établis depuis l'époque de Latreille et essai d'une nouvelle classification jusqu'aux genres exclusivement. Annales de la Societé entomologique de France, Paris, (5) 8 (1): 5-30.

Bates, H. W. 1862. Contributions to an insect fauna $\mathrm{f}$ the Amazon Valley. Lepidoptera: Heliconidae. Transaction of the Linnean Society of London 23 (3): 495-566.

Billberg, G.J. 1820. Enumeratio Insectorum in Museo Gust. Joh. Billberg. [Holmiae]. Gadel, IV+138p.

BiRkit-Smith, S.J.R. 1974. Morphology of the male genitalia of Lepidoptera II. Monotrysia, Zeugloptera, and discussion. Entomologica scandinavica, Copenhagen, 5: 161-183.

BlANCHARD, É. 1845. Histoires des Insectes, traitant de leurs mouers et de leurs métamorphoses en géneral, et comprenant une nouvelle classification fondée sur leurs rapparts naturels 2. Paris, Didot, IV+524p.

BlANCHARD, É. 1850. Sixième ordre Lépidoptères, p. 417-560,. In: F.L. Castelnau (Ed.). Histoire naturelle des Animaux articulés 3 (2). Paris, Societé Bibliophile.

BlanChard, É. 1852-1854. Orden VI. Lepidopteros. In: C. GaY (Ed.). Historia fisica y política de Chile según documentos adquiridos en esta república durante doce años de residencia en ella y publicada bajo los auspicios del Supremo Gobierno. Zoologia 7. Paris, C. Gay, 112p. [1852], pls 1-7 [1854].

Bode, W. 1907. Die Schmetterlingsfauna von Hildesheim. Mitteilungen aus dem Roemer-Museum, Hildesheim, 22: 1-65.

Bodine, D. 1896. The taxonomic value of the antennae of the Lepidoptera. Transactions of the American Entomological Society, Philadelphia, 23: 1-56.

BÖRNER, C. 1904. Zur Systematik der Hexapoden. Zoologischer Anzeiger, Leipzig, 27: 511-533.

BÖRNER, C. 1920. Lepidóptera [sic], p. 328-355. In: P. BROHMER (Ed.). Fauna Deutschland, ein Bestimmungsbuch unserer heimischen Tierwelt. Leipzig, Verlag von Quelle \& Meyer, $2^{\text {nd }}$ ed.

BÖRNER, C. 1925. Lepidóptera [sic], p. 358-387. In: P. BROHMER (Ed.). Fauna Deutschland, ein Bestimmungsbuch unserer heimischen Tierwelt. Leipzig, Verlag von Quelle \& Meyer, $3^{\text {rd }}$ ed.

BÖRNER, C. 1932. Lepidóptera [sic], p. 369-404. In: P. BROHMER (Ed.). Fauna Deutschland, ein Bestimmungsbuch unserer heimischen Tierwelt. Leipzig, Verlag von Quelle \& Meyer, $4^{\text {th }}$ ed.

BÖRNER, C. 1939. Die Grundlagen meines Lepidopterensystem. Verhandlungen des VII internationalen Kongress fuer Entomologie, Berlin, 2, p. 1372-1424.

Börner, C. 1949. Lepidóptera [sic], p. 382-421. In: P. BROHMER 
(Ed.). Fauna Deutschland, ein Bestimmungsbuch unserer heimischen Tierwelt. Leipzig, Verlag von Quelle \& Meyer, $6^{\text {th }}$ ed.

Boisduval, J.B.A.D. De \& J. Leconte. 1829-[1837]. Histoire générale et iconographique des Lépidoptéres et des Chenilles de l'Amerique septentrionale. Paris, Roret, 228p.

BoIsduval, J.B.A.D. DE. 1832-1843. Icones historique des Lépidoptères d'Europe, nouveaux ou peu connus. Collection avec figures coloriées des Papillon d'Europe novellement découverts. Ouvrage formant le complémment de tous les auteurs inconographes. Paris, Méquignon-Marvis, Crochard, Roret, 1, 251p., 2, 192p.

BoIsDuval, J.B.A.D. DE. 1836. Histoire naturelle des Insectes. Spécies géneral des Lépidoptères. Paris, Librairie Encyclopédique de Roret, 1, 4+XII+690+6p.

Boisduval, J.B.A.D. DE. 1840. Genera et Index methodicus Europaeorum Lepidopterorum. Paris, Roret, IX+238p.

Bourgogne, J. 1951. Ordre des Lépidoptères. Lepidoptera Linné, 1758; Glossata Fabricius, 1775, p. 174-448. In: P.-P. Grassé (Ed.). Traité de Zoologie. Anatomie, Systématique, Biologie. Paris, Masson et Compagnie Editeurs, vol. 10 (1), X+978p.

Brauer, F.M. 1885. Systematisch-zoologische Studien. Sitzberichte der Kaiserlichen Akademie der Wissentschaften. Philosophische-historische Classe, Wien, 91 (1): 237-413.

BRIDGES, C.A. 1984. Lepidoptera: Rhopalocera. Notes FamilyGroup Names. Urbana, Author, vol. 1, p. 1-7, vol. 2, p. 1-3, vol. 3, p. 1-7.

BRIDGES, C.A. 1988. Catalogue of Family-Group and GenusGroup Names (Lepidoptera: Rhopalocera). Urbana, Author, vol. 1, p. 1-8, vol. 2, p. 1-3, vol. 3, p. 1-4, vol. 4, p. 1-141, vol. 5, p. 1-33, vol. 6, p. 1-68, vol. 7, p. 1-18, vol. 8 , p. 1-61, vol. 9, p. 1-20, vol. 10, p. 1-8.

BRock, J.P. 1971. A contribution towards an understanding of the morphology and phylogeny of the Ditrysian Lepidoptera. Journal of natural History, London, 5: 29-102.

BRown JR., K.S. 1996. Diversity of brazilian Lepidoptera: history of study, methods for measurement, and use as indicator for genetic, specific and system richness, p. 221-253. In: C.E. DE M. Bicudo \& N.M. Menezes (Eds). Biodiversity in Brazil. A first approach. São Paulo, USP, Instituto de Botânica, 326p.

Burmeister, H.C.C. 1829. De insectorum systemate naturali. Halle, Grunert, IV+43p.

Burmeister, H.C.C. 1835-1839. Handbuch der Entomologie 2. Berlin, Reimer, 756p.

BurmeIster, H.C.C. 1878. Description physique de le République Argentine d'après des observations personelles et étrangeres 5. Lepidoptères. Premiere partie, Contenant les diurnes, crépusculaires et bombycoïdes. Buenos Aires, P.E. Coni, Paris, F. Savy; Halle, E. Anton. VI+526p.

Carter, D.J. \& N.P. Kristensen 1999. 3. Classification and Keys to Higher Taxa, p. 27-40. In N.P. KRISTENSEN (Ed.). Teilband 35. Lepidoptera, moths and butterflies. Evolution,
Systematics, and Biogeography. Berlin, vol. 1, X+491p. In: M. Fischer (Ed). Handbuch der Zoologie 4. Berlin, Walter de Gruyter.

Chapman, T.A. 1893. On some neglected points in the structure of the pupae of Heterocerous Lepidoptera, and their probable value in classification; with some associated observations on larval prolegs. Transactions of the Entomological Society of London, p. 97-119.

Chapman, T.A. 1895. Notes on butterfly pupae, with some remarks on the phylogenesis of the Rhopalocera Entomologist's Record and Journal of Variation, West Wickham, 6 (5):101-107, (6): 125-131; (7): 147-152.

Chapman, T.A. 1896. On the phylogeny and evolution of the Lepidoptera from a pupal and oval standpoint. Transactions of the entomological Society of London. 1896, p. 567-587.

Chapman, T.A. 1899. A classification of butterflies by their antennae. Entomologist's Record and Journal of Variation, West Wickham, 11(4): 85-89; (5): 122-129.

Chapman, T.A. 1916. Micropteryx entitled to ordinal rank; order Zeugloptera. Transactions of the entomological Society of London, 1916, p. 310-314.

Clairville, J. DE. 1798. Entomologie helvétique ou catalogus des Insects (Coléoptères) de la Suisse, ranges d'après uns nouvelle méthode, avec descriptions et figures. Zürich, Orell. 1, 149p.

Common, I.F.B. 1970. Lepidoptera (Moths and butterflies), p. 765-866. In: CSIRO (Ed.). The insects of Australia. A textbook for students and research workers. Canberra, Melbourne University Press, XIII+1029p.

Common, I.F.B. 1974. Lepidoptera (Moths and butterlies), p. 98107. In: CSIRO (Ed.). The insects of Australia. A textbook for students and research workers. Canberra, Melbourne University Press, VIII+146p.

Common, I.F.B. 1975. Evolution and classification of the Lepidoptera. Annual Review of Entomology, Palo Alto, 20: 183-203.

Comstock, J.H. 1892. The descent of the Lepidopotera. An application of the theory of natural selection to taxonomy. Proceedings of the American Association for the Advancement of Science, Cambridge, 61: 199-200.

Сомsтоск, J.H. 1892. [Note on the suborder of Lepidoptera: Frenatae and Jugatae]. Zoe, San Diego, San Francisco, 3 (1): 84-86

Cомsтоск, J.H. 1893. Evolution and taxonomy, an essay on the application of the theory of natural selection in the classification of animals and plants, illustrated by a study of the evolution of the wings of insects and by a contribution to the classification of the Lepidoptera, p. 95-113. In: WILDER (Ed.). The wilder quarter-century book. Ithaca, The Quarter-Century Book.

Comstock, J.H. \& A.B. Сомsтоск. 1895. A Manual for the Study of Insects. Ithaca, Comstock Publishing, 701p.

Comstock, J.H. \& A.B. Сомsтоск. 1904. A Manual for Study

Revista Brasileira de Zoologia 23 (1): 1-41, março 2006 
Insects. Ithaca, Comstock Publishing, $5^{\text {th }}$ ed., 701p.

Сомзтоск, J.H. 1918. The wings of insects. Ithaca, The Comstock Publishing, 430p.

Comstock, J.H. 1940. Introduction to Entomology. Ithaca, Comstock Publishing, $9^{\text {th }}$ ed., XIX+1064p.

Comstock, W. Рн. 1944. Insects of Puerto Rico and the Virgin Islands. Rhopalocera or butterflies. Scientific Survey of Porto Rico and the Virgin Islands 12 (4): 419-622.

Covell, C.V.O.1999. The butterflies and moths (Lepidoptera) of Kentucky, an annotated checklist. Kentucky State Nature Presserves Commission, Scientific and Technichal Series, Frankfort, 6: 1-XIV, 1-220.

D'AlmeidA, R.F. 1932. Quelques legères rémarques sur plusieurs groupes supérieurs des lépidoptères américains. Lambillionea, Bruxelles, 32 (8-9): 166-171.

D'Almeida, R.F. 1937. Excursão scientífica aos rios Cuminá e Trombetas. Memórias do Instituto Oswaldo Cruz, Rio de Janeiro, 32 (2): 235-298.

D'Almeida, R.F. 1966. Catálogo dos Papilionidae americanos. São Paulo, Sociedade Brasileira de Entomologia, 366p.

Dana, J.D. 1864. The classification of animals based on the principle of cephalization. No. II. Classifications of insects. The American Journal of Science and Arts, New Haven, (2) $37: 10-33$

DAvis, D.R. \& E.S. Nielsen. 1980. Description of a new genus and two new species of Neopseustidae from South America, with discussion of phylogeny and biological observations (Lepidoptera, Neupseustoidea). Steenstrupia, Copenhagen, 6: 253-289.

DAVIS, D.R. 1986. A new family of Monotrysian moths from Austral South America (Lepidoptera: Palaephatidae), with a phylogenetic review of the Monotrysia. Smithsonian Contributions to Zoology, Washington, 434: I-IX+1-202.

de Jong, R.; R.I. Vane-Wright \& P.R. Ackery 1996. The higher classification of butterflies (Lepidoptera): problems and prospects. Entomologica scandinavica, Copenhagen, 27: 65-101.

DoherTy, W. 1886. A list of butterflies taken in Kumaon. Journal of the Asiatic Society of Bengal, Calcutta, 55 (2): 103-140.

Dugdale, J.S. 1974. Female genital configuration in the classification of Lepidoptera. New Zealand Journal of Zoology, Wellington, 1 (2): 127-146.

Duméril, A.M.C. 1806. Zoologie analytique, ou méthode naturelle de classification des animaux, rendue plus facile à l'aide de tableaux synoptiques. Paris, Allais, p. I-XXXII, $1-344$.

DumériL, A.M.C. 1823. Considération générales sur la classe des Insects, ou l'on traite du rang que les Insects paraissent avoir occupé dans l'échelle des êtres; de leur classification, de leur distribution en genres etc. Strasbourg et Paris, Levrault, 272p.

DumérIL, A.M.C. 1823. Dictionaire des sciences naturelles, dans lequel on traite méthodiquement des differens êtres de la nature, considérés soit en eux-mêmes, d'après l'état actuel de nos connoissances, soit relativement a l'utilité qu'en peuvent retirer la médecine, l'agriculture, le commerce et les arts 26. Strasbourg F.G. Levrault.

Duméril, A.M.C. 1860. Entomologie analytique. Histoire générale, classification naturelle et méthodique des insects a l'aide des tableaux synoptiques. Paris, Didot, vols 1-2, 30+1343p.

Duponchel, P.A.J. 1844-1846. Catalogue méthodique des Lépidoptères d'Europe distribués en familles, tribus et genres avec l'exposé des caractères sur lesquels ces décisons sont fondées, et l'indication des lieux et des époques on l'on trouve chaque espèce; pour servir de complément et de rectification à l'histoire naturelle des des Lépidoptères de France. Paris, Méquignon-Marbis, $\mathrm{XXI}+523 \mathrm{p}$.

DyAr, H.G. 1893-1895. A classification of lepidopterous larvae. Annals of the New York Academy of Sciences, New York, 8: 194-232.

DyAR, H.G. 1895. A combination of two classifications of Lepidoptera. Journal of the New York Entomological Society, New York, 3: 17-26.

Dyar, H.G. 1895. Additional notes on the classification of lepidopterous larvae. Transactions of the New York Academy of Sciences, New York, 14: 49-62.

Dyar, H.G. 1895. The classification of the Lepidoptera on larval characters. The American Naturalist, a popular illustrated magazine of natural History, Philadelphia, 29: 1066-1072.

DyAr, H.G. 1895. Relationship of Pyralidae and Pterophoridae from the larvae. Entomological News, Philadelphia, 6: 3840.

FABRICIUS, J.C. 1775. Systema entomologiae, sistens insectorum classes, ordines, genera, species, adiectis synonymis, locis, descriptionibus, observationibus. Flensburgi et Lipsiae, Korte. $[\mathrm{IV}]+[\mathrm{XII}]+[\mathrm{XVI}]+832 \mathrm{p}$.

FABRICIUS, J.C. 1776. Genera insectorum eorumque characteres naturales secundum numerum, figuram, situm et proportionem omnium partium oris adjecta mantissa specierum nuper detectarum. Chilonii, Bartsch, [XIV]+310p.

FABRICIUS, J.C. 1781. Species insectorum exhibentes eorum differentias specificas, synonyma auctorum, loca natalia, metamorphosin adiectis observationibus, descriptionibus. Hamburgii et Kilonii, Carl Ernst Bohn. 2, p. 1-494; Appendix, p. 495-514; Index, p. 515-517.

FABRICIUS, J.C. 1787. Mantissa insectorum sistens species nuper detectas adiectis synonymis, observationibus, descriptionibus, emendationibus. Hafniae, Christian Gottlieb Proft. 2, [I]+382p.

FAbricius, J.C. 1793. Entomologia systematica emendata et aucta. Secundum classes, ordines, genera, species adjectis synonimis, locis, observationibus, descriptionibus. Hafniae, Christian Gottlieb Proft, Fil. et Soc. 3 (1), IV+487p. 
Fabricius, J.C. 1807. Die neuste Gattungs-Eintheilung der Schmetterlinge aus den linnéischen Gattungen Papilio und Sphinx. Magazin für Insektenkunde 6: 277-289.

FABRICIUS, J.C. 1938. Systema glossatorum secundum ordines, genera, species adiectis synonymis locis, observationibus, descriptionibus, p. [I]-XII, 13-112. In: F. Bryk. Journal Chr. Fabricius, Systema Glossatorum. Neubrandenburg, Verlag Gustav Feller. [I]-XII, [I]-XII, 13-112, 277-289.

Forbes, W.T.M. 1923. The Lepidoptera of New York and neighboring States. Primitive Forms, Microlepidoptera, Pyraloids, Bombyces. Memoir Cornell University Agricultural Experiment Station, Ithaca, 68: 1-729.

Forbes, W.T.M. 1960. Lepidoptera of New York.and neighboring States. Agaristidae through Nymphalidae, including butterflies. Memoir Cornell University Agricultural Experiment Station, Ithaca, 371: 1-188.

Fracker, S.B. 1915. The classification of lepidopterous larvae. Illinois Biological Monographs, Urbana, 2 (1): 1-161.

Fracker, S.B. 1920. The life cycle in insects. The life cycle of the Lepidoptera. Annals of the Entomological Society of America, Columbus, 13: 167-173.

Friese, G. 1969. Zur Phylogenie der älteren Teilgruppen der Lepidopteren. Bericht über die 10. Wanderversammlung Deutscher Entomologen. Tagungsberichte, Berlin, 80 (2): 203-222.

Geoffroy, E.L. 1762. Histoire abrégée des insectes qui se trouvent aux environs de Paris, dans laquelle ces animaux sont rangés suivant un ordre méthodique. Paris, Durand. 2, 690p.

Gmelin, J.F. 1790. Caroli Linné. Systema naturae per regna tria naturae, secundum classees, ordines, genera, species, cum characteribus, differentis, synonymis, locis. Editio decima tertia, aucta, reformata. Lipsiae, Georg Emanuel Ber. 1, [2]+p. 2225-3020.

Godman, F.D. \& O. SAlvin 1879. Biologia Centrali-Americana. Insecta. Lepidoptera-Rhopalocera. London, Dulau, Bernard Quaritch, 1, p. 1-56.

Gómez B., M.R. 1978. Revisión de las mas altas categorías sistemáticas de los lepidópteros y su aplicación a la Pensínsula Ibérica. Shilap, Revista de lepidopterologia, Madrid, 6 (23): 245-261.

Gómez B., M.R. 1979. Nueva (1979) revisión de las mas altas categorías sistemáticas de los lepidópteros de la P. Ibérica (1978). Shilap, Revista de lepidopterologia, Madrid, 7: 167177.

Gravenhorst, J.L.C. 1843. Vergleichende Zoologie. Breslau, Verlag von Grass, Barth, 686p.

Grote, A.R. 1889. The classification of our butterflies. Canadian Entomologist, Montreal, 21: 205-209.

Grote, A.R. 1895. Systema Lepidopterorum Hildesiae juxta opera praeliminaria, quae ediderunt Bates, Scudder, Guilielmus Mueller, Comstock, Dyar, Chapman, compositum. Mittheilungen aus dem Roemer-Museum, Hildesheim, 1:
[1-4].

Grote, A.R. 1896. System der nordamerikanischen Schmetterlinge. Mitteilungen aus dem Roemer-Museum, Hildesheim, 7: [1-4].

Grote, A.R. 1897. Die Schmetterlingsfauna von Hildesheim. Mitteilungen aus dem Roemer-Museum, Hildesheim, 8: $1-44$.

Guenée, A. 1852. Noctuélites I. In: J.B.A.D. DE Boisduval \& A. Guenée (Eds).. Histoire naturelle des Insectes. Species général des Lépidoptères. Paris, Libraire Encyclopédique de Roret, 5, XCVI+497p.

Guenée, A. 1852. Noctuélites II. In: J. B. A. D. de Boisduval \& A. Guenée (Eds). Histoire naturelle des Insectes. Species général des Lépidoptères. Paris, Libraire Encyclopédique de Roret, 6, 444p.

Guenée, A. 1852. Noctuélites III. In: J.B.A.D. DE BoIsduval \& A. Guenée (Eds). Histoire naturelle des Insectes. Species général des Lépidoptères. Paris, Libraire Encyclopédique de Roret, 7, 442p.

Guenée, A. 1854. Deltoïdes et Pyralites. In: J.B.A.D. DE BoIsDuval \& A. GuENÉE (Eds). Histoire naturelle des Insectes. Species général des Lépidoptères. Paris, Libraire Encyclopédique de Roret, 8, 448p.

Guenée, A. 1875. Statistique scientifique d'Eure-et-Loir. Lépidoptères. Chartres, Petrot-Garnier, 302p.

Guenée, A., 1887. Uranides et Phalénites. In: J.B.A.D. DE BoISDUval \& A. GuenéE (Eds). Histoire naturelle des Insectes. Species général des Lépidoptères. Paris, Libraire Encyclopédique de Roret, 9, LVI+514p.

HAase, E. 1891. Das System der Tagfalter. Deutsche entomologische Zeitschrift "Iris", Berlin, 4: 1-33.

HAECKEL, E. 1889. Natürliche Schöpfungs-Geschichte. Berlin, Verlag Georg Reimer, XXX+832p.

Handliksch, A. 1906-1908. Die fossilen Insekten und die Phylogenie der rezenten Formen. Leipzig, Verlag Wilhelm Engelmann. 1, p. XXVII+1-640 (1906); 2, p. 641-1430 (19071908).

Handlirsch, A. 1925. In: C.W.M. Schröder (Ed.) Handbuch der Entomologie. Jena, Gustav Fischer, vol 3, VIII+1202p.

HaYward, K.J. 1948. Insecta, Lepidoptera (Rhopalocera), familia Hesperiidarum, subfamiliae Pyrrhopyginarum et Pyrginarum. In: H.R. Descole (Ed.). Genera et Species Animalium Argentinorum. Buenos Aires, Guillermo Kraft. 1, [10]+389p.

HenNIG, W. 1953. Kritische Bemerkungen zum phylogenetischen System der Insekten. Beiträge zur Entomologie, Berlin, 3, Sonderheft, p. 1-85.

Hennig, W. 1986. Taschenbuch der Zoologie 3. Wirbellose II. Jena, Gustav Fischer Verlag, $4^{\text {th }}$ ed., 335 p.

Heppner, J.B. 1984. Atlas of neotropical Lepidoptera 2. Checklist: Part 1. Micropterigoidea - Immoidea. The Hague, Dr. W. Junk Publishers. XXVII+112p.

Heppner, J.B. (Ed.) 1995. Atlas of neotropical Lepidoptera 3. 
Checklist: Part 2. Hyblaeoidea - Pyraloidea - Tortricoidea. Gainesville, Scientific Publishers, LIV+243p.

Heppner, J.B. 1996. Atlas of neotropical Lepidoptera 5B. Checklist: Part 4B. Drepanoidea - Bombycoidea Sphingoidea. Gainesville, Scientific Publishers, L+87p.

Heppner, J.B. 1998. Revised family list for Lepidoptera. Lepidoptera News, Gainesville, (3): 57-62.

Heppner, J.B. 1998. Classification of Lepidoptera. Part 1. Introduction. Holarctic Lepidoptera, Gainesville, 5 (Suppl. 1): I-IV, 1-148.

Heppner, J.B. 2003. Lepidoptera of Florida. Part 1. Introduction and catalog. Arthropods of Florida and neighboring land areas. Gainesville, Florida Department of Agriculture \& Consumer Services, vol. 17, X+670p.

Herrich-Schäffer, A. 1840. II Animalia articulata. Classis I. Insecta, p. 45-151. In: A. E. FÜRNROHR (Ed.). Naturhistorische Topographie von Regensburg 3. Regensburg, Verlag von G.J. Manz.

Herrich-SchäfFer, G.A.W. 1845. Systematische Bearbeitung der Schmetterlinge von Europa, als Text, Revision und Supplement zu J. Huebners Sammlung europäischer Schmetterlinge. Regensburg, Verlag von G.J. Manz, vol 2, 450p.

Herrich-SChäFFER, G.A.W. 1849. Systematische Bearbeitung der Schmetterlinge von Europa, als Text, Revision und Supplement zu J. Huebners Sammlung europäischer Schmetterlinge. Regensburg, Verlag von G.J. Manz, vol. 4, 288p.

HerRich-SChÄFFER, G.A.W. 1849. Zur Literatur der Schmetterlingskunde. Correspondez-Blatt des zoologisch-mineralogischen Vereines in Regensburg 3: 26-30, 41-47, 53-56, 76 79, 141-144, 151-160, 162-173.

Herrich-SCHÄFFer, G.A.W. 1852. Zur Synonymik in der Schmetterlingskunde. Correspondenz-Blatt des zoologisch-mineralogischen Vereines in Regensburg 6: 17-32, 188-194.

HerRICH-SCHÄFFER, G.A.W. 1850-[1858]. Sammlung neuer oder wenig bekannter aussereuropäischer Schmetterlinge. Regensburg, G. L. Manz. 1, 84p.

Herrich-SCHÄFFER, G.A.W. 1862-1863. Schmetterlinge aus Cuba. Correspondez-Blatt des zoologisch-mineralogischen Vereines in Regensburg 16 (6/8): 118-120, (10): 141-143, (11): 156-157, (12): 174-180; 17 (9): 138-143, (10): 147-150.

Heymons, R. 1915. Sechsfüssler, Insekten (Hexapoda, Insecta), p. 36-326. In: O. zUR STRASSEN (Ed.). Brehms Tierleben. Allgemeine Kunde des Tiereichs. 2. Die Vielfüssler, Insekten und Spinnenkerfe. Leipzig und Wien, Bibliographisches Institut.

Hinton, H.E. 1946. On the homology and nomenclature of the setae of lepidopterous larvae, with some notes on the phylogeny of the Lepidoptera. Transactions of the royal entomological Society of London 97: 1-37.

Holland, W.J. 1898. The Butterfly Book. A popular guide to a knowledge of the butterflies of North America. New York,
Doubleday \& McClure, XX+382p.

Holland, W.J. 1945. The Butterfly Book. A popular guide to a knowledge of the butterflies of North America. New York, Doubleday, Doran, XII+424p.

Holloway, J.D.; J.D. Bradley \& D.J. Carter. 1987. CIE Guides to insects of importance to man. 1. Lepidoptera. London, CAB International Institute of Entomology, IV+262p.

HÜBNER, J. 1816-[1819]. Verzeichniss bekannter Schmettlinge [sic]. Augsburg, Jacob Hübner, (1), p. 1-16 (1816), (2-8), p.17128 [1819].

Ihering, H. von. 1929. Phylogenie und System der Tagfalter. Entomologische Rundschau, Stuttgart, 46 (8): 29-30, (9): 33-34, (11): 41-42.

ImMS, A.D. 1930. A General Text-book of Entomology. London, Methun, $2^{\text {nd }}$ ed., $X+703 p$.

JABLONSKY, C.G. 1783. Natursystem aller bekannten in- und ausländischen Insekten als eine Fortsetzung der von Büffonischen Naturgeschichte, nach dem System des Ritters Carl von Linne bearbeitet. Berlin, Joachim Pauli, $216 p$.

Jordan, H.E.K. 1907-1908. I. Familie: Papilionidae, Segelfalter, p. 11-45, 48-51. In: A. SEITZ (Ed.). Die Gross-Schmetterlinge der Erde 5. Die Gross-Schmetterlinge des Amerikanischen Faunengebietes. Stuttgart, Alfred Kernen,1141p.

Karsch, F.A.F. 1898. Giebt es ein System der recenten Lepidopteren auf phyletischer Basis? Entomologische Nachrichten, Berlin, 24: 296-303.

Kirby, W. \& W. SPenCe 1826. An introduction to Entomology or elements of the Natural History of Insects. London, Longmann. 4, 634p.

Kirby, W.F. 1904-1908. [Notes], p. 1-32 [1900]. In: J. Hübner (Ed.). Sammlung exotischer Schmetterlinge 3. Reedição de W. F. Kirby \& P. Wytsman. Bruxelas, V. Verteneuil \& L. Desmet.[3]+172p.

KirbY, W.F. 1908-1912. Zuträge zur Sammlung exotischer Schmetterlinge bestehend in Bekündigung einzelener Fliegmuster neuer oder rarer nichteuropäischer Gattungen von Jakob Hübner 1818-1825 (Tafeln 1-113) Carl Geyer 1832-1837 (Tafeln 104-172). New English facsimile edition with 172 colored plates with introduction, and systematic and alphabethical indices. Bruxelas, V. Verteneuil \& L. Desmet, Reedição de W.F. Kirby \& P. Wytsman, [4]+II+101p.

Kiriakoff, S.G. 1946. Notes systématiques. II. Position systématique des Lepidoptères et les divisions primaires de l'ordre. Lambillionea, Bruxelas, 46: 21-26.

Kiriakoff, S.G. 1946. Notes systématiques. V. Classifications récents des Lépidoptères. Lambillionea, Bruxelas, 46: 11116.

Kiriakoff, S.G. 1946. On the systematical position of the lepidopterous family Hesperiidae. Biologisch Jaarboek, Antwerpen, 13: 288-292.

Kiriakoff, S.G. 1948. A classification of the Lepidoptera and 
related groups with some remarks on taxonomy. Biologisch Jaarboek, Antwerpen, 15: 118-143.

Kiriakoff, S.G. 1952. L'usage des categories taxonomiques intermediaires dans la classification phylogenetique des Lepidopteres. Annales de la Société royale zoologique de Belgique, Bruxelas, 83: 87-106.

Kiriakoff, S.G. 1955. Le système phylogénétique: Principe et méthodes. Bulletin \& Annales de la Société entomologique de Belgique, Bruxelas, 91: 156-158

Косн, G. 1856. Die Schmetterlinge des südwestlichen Deutschlands, insbesondere der Umgebung von Frankfurt, Nassau und der Hessischen Staaten, nebst Angabe der Fundorte und Flugplätze. Zum Gebrauche für Sammler bei Excursionen. Cassel, Fischer, 20+498p.

Krausse A. \& M. Wolff 1919. Eine Übersicht über die bisher aufgestellten fossilen und rezenten Insektenordnungen. Archiv für Naturgeschichte, Berlin, 86A (3): 151-171.

Kristensen, N.P. \& E.S. Nielsen. 1981. Intrinsic proboscis musculature in non-ditrysian Lepidoptera-Glossata: Structure and phylogenetic significance, p. 300-304. In: L. Cederholm (Ed.). Advances in insect systematics and phylogeny. Entomologica scandinavica, Copenhagen, (Suppl. 15).

Kristensen, N.P. \& E. Nielsen. 1983. The Heterobathmia life history elucidated: Immature stages contradict assignement to suborder Zeugloptera (Lepidoptera, Lepidoptera). Zeitschrift für zoologische Systematik und Evolutions-Forschung, Hamburg und Berlin, 21: 101-124.

Kristensen, N.P. 1984. Studies on the morphology and systematics of primitive Lepidoptera (Insecta). Steenstrupia, Copenhagen, 10: 142-191.

Kristensen, N.P. 1985. Katalog over de danske Sommerfugle. Catalogue of the Lepidoptera of Denmark. Entomologiske Meddelelser, Copenhagen, 52: 6-20.

Kristensen, N.P. \& A.W. Skalski. 1999. 2. Phylogeny and Paleontology, p. 7-25. In: N.P. Kristensen (Ed.). Teilband 35. Lepidoptera, Moths and Butterflies. Evolution, Systematics, and Biogeography. vol. 1, X+491p. In: M. Fischer (Ed). Handbuch der Zoologie 4. New York, Walter de Gruyter.

Kristensen, N.P. 1999. 4. The Non-Glossatan Moths, p. 41-49. In: N.P. KRISTENSEN (Ed.). Teilband 35. Lepidoptera, Moths and Butterflies. Evolution, Systematics, and Biogeography. vol. 1, X+491p. In: M. FISCHER (Ed). Handbuch der Zoologie 4. New York, Walter de Gruyter.

Kristensen, N.P. 1999. 5. The Homoneurous Glossata, p. 51-63. In: N.P. KRISTENSEN (Ed.). Teilband 35. Lepidoptera, Moths and Butterflies. Evolution, Systematics, and Biogeography. vol. 1, X+491p. In: M. FISCHER (Ed). Handbuch der Zoologie 4. New York, Walter de Gruyter.

KüPpers, P.V. \& W. SPeidel V-. 1980. Die systematische Stellung der Neospeustidae. Atalanta, Marktleuthen, 11 (1): 55-65.

KuITJEN, P.J. 1974. On the occurrence of a hitherto unknwon wing-thorax coupling mechanism in Lepidoptera. Netherland Journal of Zoology, Leiden, 24: 319-320.

Kuznetzov, N.Y. 1915. Fauna de la Russia et des pays limitrophes fondée principalemente sur les collections de Musée Zoologique de l'Academie Impériale des Sciences de Petrograd. São Petersburgo, 336 p.

Kuznetzov, N.Y. 1967. Fauna of Russia and adjacent countries. Lepidoptera. Jerusalem, Published for the U.S. Department of Agriculture and the National Science Foundation, Translated from the russian by the Israel Program for Scientific Translations, vol. 1, 4+305p.

KuZNETZOV, V.I. \& A.A. STEKOLNiKov 1978. The system and evolution of infraorders in the Lepidoptera (Micropterigomorpha, Papilionomorpha) treated on the base of functional morphology of the genitalia. Entomologicheskoe Obozrenie, São Petersburgo, 57: 870-890.

KuZnEtzov, V.I. \& A.A. SteKolnikov 1979. The system and evolution of infraorders in the Lepidoptera (Micropterigomorpha Papilionomorpha) in the light of the functional morphology of the genitalia. Entomological Review, New York, 57: 609613.

Laicharting, J.N. von. 1781-1784. Verzeichniss und Beschreibung der Tyroler Insecten. Zürich, Füessly, vol 1, 283p.; vol. 2, 176p.

LAMAS, G. 2004. Checklist: Part 4A. Hesperioidea - Papilionoidea. In: J.B. Heppner (Ed.). Atlas of Neotropical Lepidoptera 5A. Gainesville, Association for Tropical Lepidoptera, XXXVI+439p.

LAMEERE, A. 1931. Abrégé de la classification zoologique. Recueil de l'Institut zoologique Torley-Rousseau, Bruxelas, 3 (2): $165-283$.

Lameere, A. 1936. Évolution des Lépidoptères. Bulletin \& Annales de la Societé entomologique de Belgique, Bruxelas, 76: 407-413.

Lameere, A. 1938. Précis de Zoologie. Lépidoptères. Recueil de l'Institut zoologique Torley-Rousseau, Bruxelas, 5: 162229.

Latreille, P.A. 1796. Précis des caractères génériques des Insectes, disposés dans un ordre naturel par le citoyen Latreille. Brive, Bourdeaux, 14+210+5p.

LATREILLE, P.A. 1802. Histoire naturelle, générale et particulière des Crustacés et des Insectes. Ouvrage faisant suite aux oeuvres de Leclerq de Buffon et partie du cours complet d'Histoire naturelle rédigé p. C. S. Sonnini. Paris, F. Dufart, vol. 2, 380p.

LATREILLE, P.A. 1803. Histoire naturelle, générale et particulière des Crustacés et des Insectes. Ouvrage faisant suite aux oeuvres de Leclerq de Buffon et partie du cours complet d'Histoire naturelle rédigé p. C. S. Sonnini. Paris, F. Dufart, vol. 5, 406p.

LATREILLE, P.A. 1805. Histoire naturelle, générale et particulière des Crustacés et des Insectes. Ouvrage faisant suite aux oeuvres de Leclerq de Buffon et partie du cours complet 
d'Histoire naturelle rédigé p. C. S. Sonnini. Paris, F. Dufart, vol. 14, 432p.

Latreille, P.A. 1806. Genera Crustaceorum et Insectorum secundum ordinem naturalem in familias disposita, iconibus exemplisque plurimis explicata. Paris et Argentorat, König, vol. 1, 18+302 p.

Latreille, P.A. 1809. Genera Crustaceorum et Insectorum secundum ordinem naturalem in familias disposita, iconibus exemplisque plurimis explicata. Paris et Argentorat, König, vol. 4, 399p.

Latreille, P.A. 1810. Considérations génerales sur l'ordre naturel des animaux composant les classes des Crustacés, des Arachnides et des Insectes avec un tableau méthodique de leurs genres disposés en familles. Paris, Schoell, 444p.

Latreille, P.A. 1817. Les Crustacés, les Arachnides et les Insectes. In G.L. Ch. D. Cuvier (ED). Le Règne Animal 3. Paris, Librairie Deterville, 29+653p.

Latreille, P.A. 1825. Familles naturelles du règne animal exposées succinctement et dans un ordre analytique avec $\mathrm{l}^{\prime}$ indication de leurs genres. Paris, J.B. Baillière, 570p.

Latreille, P.A. 1829. Le dixième ordre des insectes. Celui des Lépidoptères. (Lepidoptera, Glossata Fab.), p. 366-424. In G.L. Ch. D. Cuvier (Ed.). Le Règne Animal 5. Paris, Librairie Dterville.

LEACH, W.E. 1815. Entomology, p. 57-172. In: Brewster (Ed.). Edinburgh Encyclopaedia 9 (1).

LeACH, W.E. 1816. Entomology, p. 646-758. In: Brewster (Ed.). Edinburgh Encyclopaedia 8 (2).

LEACH, W. 1819. The modern method of arranging the classes Crustacea, Myriapoda, Spiders, Mites and Insects, from their affinities and structure. In: G. Samouelle (Ed.). The entomologists useful compendium, or an introduction to the knowledge of British Insects comprising the best means of obtaining and preserving them, and a description of the apparatus generally used; together with the genera of Linné and the modern method of arranging the classes etc. London, Thomas Boys, 496p.

LEACH, W.E. 1830. Entomology, p. 57-172. In: Brewster (Ed.). Edinburgh Encyclopaedia (4) 9.

Lederer, J. 1853. Versuch die europäischen Lepidopteren in möglichst natürliche Reihenfolgezu stellen. Verhandlungen des zoologisch-botanischen Vereins in Wien 2: 14-54.

Le Marchand, S. 1945. Les Microlépidoptères. Classification des Tineina. Revue française de Lépidopterologie, Paris, 10: 94-111, 125-144.

Lindsey, A.W. 1921. The Hesperioidea of America North of Mexico. University of Iowa Studies in Natural History, Iowa, 9 (4): 1-114.

Lindsey, A.W. 1925. The types of hesperioid genera. Annals of the Entomological Society of America, Lanham, 18 (1): 75-106.

LINNAEUS, C. 1758. Systema naturae per regna tria naturae, secundum classes, ordines, genera, species, cum characteribus, differentiis, synonymis, locis 1 . Holmiae, Laurentius Salvius, Editio Decima, reformata, IV+823+[1]p.

LinNaeus, C. 1767. Systema naturae 1 (2). Holmiae, Laurentius Salvius, Editio duodecima reformata, [II]+533-1328+[36] p.

LuCAS, P.H 1864. Histoire naturelle des Lépidoptères exotiques. Paris, F. Savy Libraire-Editeur, $2^{\mathrm{a}}$ ed., [4]+IV+156p.

LuCAS, P.H. 1864. Histoire naturelle des lépidoptères d'Europe. Paris, Savy Libraire-Editeur, $2^{\text {a }}$ ed., 292p.

Mеүrick, E. 1895. Handbook of british Lepidoptera. London, Macmillan, 843p.

MeYrick, E. 1928. A revised Handbook of british Lepidoptera. London, Watkins \& Doncaster, 914p.

Minet, J. 1983. Étude morphologique et phylogénique des organs tympaniques des Pyraloidea. 1 - Généralités et homologies (Lep. Glossata). Annales de la Societé entomologique de France (n. s), Paris, 19 (2): 175-207.

Minet, J. 1984. Contribution a l'analyse phylogénétique des Néolépidoptères (Lepidoptera, Glossata). Nouvelle Revue d'Entomologie (n. s), Fontenay-sous-Bois, 1 (2): 139-149.

Minet, J. 1986. Ebauche d'une classification moderne de l'ordre des Lépidoptères. Alexanor, Paris, 14 (7/8): 291-313.

Minet, J. 1991. Tentative reconstruction of the ditrysian phylogeny (Lepidoptera: Glossata). Entomologica Scandinavica, Copenhagen, 22 (1): 69-95.

Minet, J. 1994. The Bombycoidea: Phylogeny and higher classification (Lepidoptera: Glossata). Entomologica Scandinavica, Copenhagen, 25 (1): 63.

Newman, E 1834. Attempted division of British insects into natural orders. Entomological Magazine, London, 2 (4): 379430 .

Newman, E. 1870. Concerning the classification of butterflies. Entomologist, London, 5: 33-42.

Niculescu, E.V. 1970. Aperçu critique sur la systématique et la phylogénie des lépidoptères. Bulletin de la Societé Entomologique de Mulhouse 1970 (jan.-févr.): 1-16.

Niculescu, E.V. 1987. L'evolution de l'Ordre Lepidoptera et ses lingnees phyletiques sous-ordinales. Shilap, Revista de lepidopterologia, Madri, 15: 107

NiculesCu, E.V. 1989. Erreurs à rectifier dans la taxonomie et la classification de l'ordre des Lépidoptères (Lepidoptera). Deutsche entomologische Zeitschrift (N. F.), Berlin, 36 (4/ 5): 335-345.

NielSEn, E.S. 1985. Primitive (non-ditrysian) Lepidoptera of the Andes: diversity, distribution, biology and phylogenetic relationship. Journal of Research on the Lepidoptera, Santa Barbara, (Suppl. 1): 1-15.

NielSEN, E.S. 1989. Phylogeny of major lepidopteran groups. In: B. Fernhelm; K. Bremer \& H. Jörnvall (Eds). The Hierarchy of Life. London, Elsevier, p. 281-294.

Nielsen E.S. \& I.F.B. Common 1991. Lepidoptera (Moths and butterflies), p. 817-915. In: CSIRO (Ed.). The Insects of Australia. Carlton, Melbourne University Press, 1137p.

Revista Brasileira de Zoologia 23 (1): 1-41, março 2006 
Nielsen E.S. \& N.P. Kristensen 1996. The Australian moths family Lophocoronidae and the basal phylogeny of the Lepidoptera-Glossata. Invertebrate Taxonomy, Collingwood, 10: 1199-1302.

PACKARD, A.S. VI-1895. On the phylogeny of the Lepidoptera. Zoologischer Anzeiger, Leipzig, 18: 228-236.

PACKARD, A.S. VII-1895. On a new classification of the Lepidoptera. American Naturalist, Philadelphia, 29: 636647, 788-803.

PACKARD, A.S. 1895. Monograph of the Bombycine Moths of America North of Mexico, including their transformations and origin of the larval markings and armature 1. Family 1.- Notodontidae. Memoirs of the National Academy of Sciences, Washington, 7: 3-290.

Parra H., M.L.; J.I. Vargas C. \& M. Tabares P. 2000. Mariposas de Manizales. Manizales, Instituto para la Ciencia, 117p.

Plöтz, C. 1886. System der Schmetterlinge. Mittheilungen aus dem naturwissentschaflichen Verein für NeuVorpommern und Rügen, Greifswald, 17: 485-528.

Poey, F. 1846. Catálogo metódico y descriptivo de las mariposas de la Isla de Cuba. Memorias de la Real Sociedad Económica de La Habana (2) 2 (3): 174-177.

Poole, R.W. 1996. Lepidoptera, p. 605-1121. In R.W. Poole \& P. Gentili (Eds). Nomina Insecta Nearctica. A Check List of the Insects of North America. Rockville, Eos, vol. 3, 1142p.

Powell, J.A.; C. Mitter \& B. Farrell 1999. Evolution of Larval Food Preferences in Lepidoptera, p. 403-422. In: N.P. Kristensen (Ed.). Teilband 35. Lepidoptera, Moths and Butterflies. Evolution, Systematics, and Biogeography. vol. 1, X+491p. In: M. FIsCHER (Ed). Handbuch der Zoologie 4. New York, Walter de Gruyter.

Prieto, A.V. \& L.M. Constantino. 1996. Abundancia, distribución y diversidad de mariposas (Lep. Rophalocera [sic]) en el Río Tatabro, Buenaventura (Valle - Colombia). Boletin del Museo de Entomologia de la Universidad del Valle, Cali, 4 (2): 11-18.

PuyséGuR, K. DE 1938. Classification des Rhopalocères. Riviera Scientifique, Nice, 25: 3-13.

Rafinesque, C.S. 1815. Analyse de la Nature ou Tableau de l'Univers et des Corps organisés. Palermo, Autor, 224p.

Rambur, P. 1942. Faune entomologique de L'Andalousie. Madri, Instituto Español de Entomologia, $2^{\mathrm{a}}$ ed., XIX+336p.

Raymundo da Silva, B. 1907. Lepidópteros do Brasil. Contribuição para a História Natural. Rio de Janeiro, Imprensa Nacional, 182p.

Razowski, J. 1974. Phylogeny and classification of Lepidoptera. Acta zoologica cracoviensia, Cracóvia. 19: 1-18.

Retzius, A.J. 1783. Caroli De Geer genera et species insectorum et generalissimi auctoris scriptis extraxit, digessit, latine quoad partem reddidit, et terminologiam insectorum Linneanam addidit. Lipsiae, Cruse, VI+220p.

Reuter, E.R. 1896. Über die Palpen der Rhopalocera. Ein Beitrag zur Erkenntnis der verwandtschaftlichen Beziehungen unter den Tagfaltern. Acta Societatis Scientiarum Fennicae, Helsingfors, 22 (1): I-XVI, 1-577.

Reuter, E.R. 1898. On a new classification of the Rhopalocera. Entomologist's Record and Journal of Variation, West Wickham, 10 (2): 25-26; (3): 75-77; (4): 95-98.

Richards, O.W. \& R.G. Davies 1957. In: A.D. Imms (Ed.). A General Textbook of Entomology. Including the Anatomy, Physiology, Development and Classification of Insects. London, Methuen, $9^{\text {th }}$ ed., X+886p.

Roemer, J.J. von. 1789. Genera insectorum Linnaei et Fabricii iconibus illustrata. Vitoduri, Heinrich Steiner et Socios, VIII+86+[3]p.

SCHLuga, J.B. 1767. Primae lineae cognitionis Insectorum cum figuris aeneis. Viennae, Kraus, $1+47+4 \mathrm{p}$.

Sсносн, G. 1884. Über die Gruppierung der InsektenOrdnungen. Mittheilungen der Schweizerischen Entomologischen Gesellschaft, Bern, 7: 34-36.

Scoble, M.J. 1989. Classification of the Lepidoptera, p. 11-45. In: A.M. EMmET \& J. HeAD (Eds). The Moths and Butterflies of Great Britain and Ireland. Colchester, Harley Books, vol. 7, part 1, X+370p.

Scoble, M.J. 1992. The Lepidoptera: Form, Function and Diversity. London, Oxford University Press, IX+352p.

Scopoli, J.A. 1763. Entomologia Carniolica exibens Insecta Carnioliae indigena et distributa in ordines, genera, species, varietates, methodo Linneana. Vindobonae, Trattner, XXXVI+420p.

Snellen, P.C.T. 1882. Aanteekeningen over Afrikaansche Lepidoptera. Tijdschrift voor Entomologie, Leiden, 25: 215234.

Speidel, W. 1977. Ein Versuch zur Unterteilung der Lepidoptera in Unterordnungen. Atalanta, Marktleuthen, 8 (2): 119-121.

SpINolA, M.M. 1852. Tavola sinotttica dei generi spettanti alla classe degli Insetti arthroidignati Hemiptera Linn. Latr., Rhyngota Fabr., Rhynchota Burm. Memoire di Matematica e di Fisica della Società italiana delle Science Residente in Modena, Modena, 25 (1): 43-100.

Spuler, A. 1908. Die Schmetterlinge Europas. Stuttgart, E. Schweiserbartsche Verlagsbuchhandlung, vol. 1, CXXVIII+385p.

Spuler, A. 1910. Die Schmetterlinge Europas. Stuttgart, E. Schweiserbartsche Verlagsbuchhandlung, vol. 2, 526p.

Stainton, H.T. 1857. A Manual of British Butterflies and Moths. London, van Voorst, vol. 1, 338p.

Stainton, H. T. 1859. A Manual of British Butterflies and Moths. London, van Voorst, vol. 2, 475+11p.

STEPHENS, J.F. 1828. Illustrations of British Entomology; or, a synopsis of indigenous insects: containing their generic and specific distinctions; with an account of their metamorphoses, times of appearance, localities, food, and economy, as far as practicable. Haustellata. London, Baldwin and Cradock, vol. 1, [2]+150+[2]p; vol. 2, 202p; vol. 3, 333p. 
STEPHENS, J.F. 1834. Illustrations of British Entomology; or, a synopsis of indigenous insects: containing their generic and specific distinctions; with an account of their metamorphoses, times of appearance, localities, food, and economy, as far as practicable. Haustellata. London, Baldwin and Cradock, vol. 4, 431p.

Stichel, H.F.E.J. 1910. Lepidoptera Rhopalocera. Fam. Riodinidae. Allgemeines - Subfamilie Riodininae, p. 1-238. In: P. WyTsman (Ed.). Genera Insectorum 112A.

Stoll, C. 1782. Proeve van eene Rangschikkinge der Donsyleugelige Insecten, Lepidopteren. Welker Afbeeldingen in de vier Deelen van dit Werk zyn te winden. Essai d'un ordre systématique des insectes a ailes farineuses. Lepidooptera. Réprésentés dans les quatre volumes de cet ouvrage, 29p. In: P. Cramer. De Uitlandische Kapellen voorkomende in de drie Waereld-Deelen Asia, Africa en America. Papillons exotiques des trois parties du monde l'Asie, l'Afrique et l'Amerique 4. Amsteldam, J.S. Balde; Utrecht, Barthelmy Wild, 252p.

Swainson, W. 1827. A sketch of the natural affinities of the Lepidoptera Diurna of Latreille. Philosophical Magazine, Abingdon, (2) 1 (3): 180-188.

Thunberg, C.P. 1791. Characteres Generum Insectorum, variis cum adnotationibus. Gottingae, Dietrich, 48p.

Tillyard, R.J. 1918. The Panorpoid complex. Part I. The wingcoupling apparatus, with special reference to the Lepidoptera. Proceedings of the Linnean Society of New South Wales, Elizabeth Bay, 43: 296.

Turner, A.J. 1947. A review of the phylogeny and classification of the Lepidoptera. Proceedings of the Linnean Society of New South Wales, Sidney, 71: 303-338.
TutT, J.W. 1896. British Butterflies, being a popular handbook for young students and collectors. London, George Gill and Sons, 476p.

TutT, J.W. 1897. On the classification of the Lepidoptera. Entomologist's Record and Journal of Variation, West Wickham, 9: 194-196.

TutT, J.W. 1899. A natural history of the British Lepidoptera. A textbook for students and collectors 1. London, Swan Sonnenschein, 560p.

WALKER, F. 1854. List of the Specimens of Lepidopterous Insects in the Collection of the British Museum. London, Trustees of the British Museum, vol. 1, 278p.

Wallengren, H.D.J. 1872. Skandinaviens Pyralider och Choreutider beskrifne. Öfversight kungliga VetenskapsAkademiens Förhandlingar, Stockholm, 28: 961-1060.

WARren, B.C.S. 1947. Some principles of classification in Lepidoptera, with special reference to the butterflies. Entomologist, London, 80 (1012): 208-217; (1013): 235241; (1014): 262-268; (1015): 280-282.

Westwood, J.O. 1838. An introduction to the modern classification of insects, founded on the natural habits and corresponding organisization of the different families. London, Longman, vol. 1, XIV+462p.

WeSTWOOD, J.O. 1839-1840. An introduction to the modern classification of insects, founded on the natural habits and corresponding organisization of the different families. London, Longman, vol. 2, 587p.

Wolff, M. \& A. Krausse. 1930. Die wirbellosen Tiere, eine systematische Übersicht. Jena, Gustav Fischer.

Zetterstedt, J.W. 1838-1840. Insecta Lapponica descripta. Lipsiae, Voss, 1140p.

Recebido em 20.V.2005; aceito em 20.I.2006. 